\title{
ANALYSIS OF STELLAR OCCULTATION DATA. II. INVERSION, WITH APPLICATION TO PLUTO AND TRITON ${ }^{1}$
}

\author{
J. L. Elliot, ${ }^{2,3}$ M. J. Person, ${ }^{2}$ AND S. Qu ${ }^{2,4}$ \\ Received 2002 December 17; accepted 2003 March 26
}

\begin{abstract}
We present a method for obtaining atmospheric temperature, pressure, and number density profiles for small bodies through inversion of light curves recorded during stellar occultations. This method avoids the assumption that the atmospheric scale height is small compared with the radius of the body, and it includes the variation of gravitational acceleration with radius. First we derive the integral equations for temperature, scale height, pressure, number density, refractivity, and radius in terms of the light-curve flux. These are then cast into summation form suitable for numerical evaluation. Equations for the errors in these quantities caused by Gaussian noise in the occultation light curve are also derived. The method allows for an arbitrary atmospheric boundary condition above the inversion region, and one particular boundary condition is implemented through least-squares fitting. When the inversion equations are applied to noiseless test data for a simulated isothermal atmosphere, numerical errors in the calculated temperature profile are less than 5 parts in $10^{4}$. Nonisothermal test cases are also presented. We explore the effects of (1) the boundary condition, (2) data averaging (in the time, observer-plane, and body-plane domains), (3) systematic errors in the zero stellar flux level, and (4) light-curve noise on the accuracy of the inversion results. A criterion is presented for deciding whether inversion would be an appropriate analysis for a given stellar occultation light curve, and limitations to the radial resolution of the inversion results are discussed. The inversion method is then employed on the light curves for the 1988 June 9 occultation by Pluto observed with the Kuiper Airborne Observatory and the 1997 November 4 occultation by Triton observed with the Hubble Space Telescope. Under the (possibly incorrect) assumption that no extinction effects are present in the occultation light curve, the Pluto inversion yields a $110 \mathrm{~K}$ isothermal profile down to approximately $1215 \mathrm{~km}$ radius, at which point a strong thermal gradient, $3.9 \pm 0.6 \mathrm{~K} \mathrm{~km}^{-1}$, abruptly appears, reaching $93 \mathrm{~K}$ at the end of the inversion. The Triton inversion yields a differently shaped profile, which has an upper level thermal gradient, $\sim 0.4 \mathrm{~K} \mathrm{~km}^{-1}$, followed by a $\sim 51 \mathrm{~K}$ isothermal profile at lower altitudes. The Triton inversion shows wavelike temperature variations in the lower atmosphere, with amplitudes of $\sim 1 \mathrm{~K}$ and wavelengths of $\sim 20 \mathrm{~km}$, that could be caused by horizontal or vertical atmospheric waves.
\end{abstract}

Key words: occultations - planets and satellites: individual (Pluto, Triton)

\section{INTRODUCTION}

Pluto and Triton both have a tenuous, predominantly $\mathrm{N}_{2}$ atmosphere that is in vapor pressure equilibrium with surface ice. The extreme sensitivity of the nitrogen vapor pressure to the temperature of the surface ice (Brown \& Ziegler 1980) means that large seasonal changes in surface pressure can accompany changes in insolation and surface albedo. Indeed, following the Voyager encounter of the Neptune system in 1989, such changes were predicted for Triton by several authors (Spencer \& Moore 1992; Hansen \& Paige 1992) and subsequently observed (Elliot et al. 1998, 2000a). Vapor pressure equilibrium also has implications for Triton's atmospheric dynamics (Ingersoll 1990). Similarly, Pluto's large orbital eccentricity $(0.25)$ would lead one to believe that its atmosphere might undergo collapse as it

\footnotetext{
${ }^{1}$ Based on observations with the NASA/ESA Hubble Space Telescope, obtained at the Space Telescope Science Institute, which is operated by the Association of Universities for Research in Astronomy, Inc., under NASA contract NAS 5-26555.

${ }^{2}$ Department of Earth, Atmospheric, and Planetary Sciences, Massachusetts Institute of Technology, 77 Massachusetts Avenue, Cambridge, MA 02139-4307.

${ }^{3}$ Department of Physics, Massachusetts Institute of Technology; and Lowell Observatory, 1400 West Mars Hill Road, Flagstaff, AZ 86001-4499.

${ }^{4}$ Department of Aeronautics and Astronautics, Massachusetts Institute of Technology, 77 Massachusetts Avenue, Cambridge, MA 02139-4307.
}

recedes from the Sun (perihelion was in 1989); however, this view has been called into question by Stansberry \& Yelle (1999), who modeled the seasonal transition between the $\alpha$ and $\beta$ phases of $\mathrm{N}_{2}$ ice on Pluto's surface and found (under certain assumptions) that its surface pressure may experience only a small change between perihelion and aphelion.

Another issue for Pluto's atmosphere that has been debated since it was first observed in the 1988 stellar occultation is whether the sharp drop (also known as a "kink" or "knee") in the light-curve flux recorded aboard the Kuiper Airborne Observatory (KAO; Elliot et al. 1989) is caused by (1) extinction from a tenuous haze layer (Elliot et al. 1989; Elliot \& Young 1992, hereinafter Paper I) or (2) a sharp thermal gradient (Eshleman 1989; Hubbard, Yelle, \& Lunine 1990b). The haze layer hypothesis has been criticized on the grounds that the atmosphere is too tenuous to support haze particles for any length of time (Stansberry, Lunine, \& Tomasko 1989). However, there is also difficulty in establishing a radiative-conductive model that can precisely reproduce the KAO light curve (D. Strobel et al. 1995, private communication). Hence it is important to glean all that we can from the KAO observations in order to establish firm requirements for physical models of Pluto's atmosphere.

Although the inversion method had been used for many years in seismology (Aki \& Richards 1980) and radio occultation data for spacecraft (Fjeldbo \& Eshleman 1965), it 
was not until the Neptune occultation of BD $-17^{\circ} 4388$ in 1968 that the inversion method was applied to stellar occultation data, by Kovalevsky \& Link (1969). The difference between the application of the inversion technique to radio occultation data and to optical occultation data is that the phase is known in the former, but only the amplitude is known in the latter. Wasserman \& Veverka (1973) investigated the uncertainties in the temperature profiles that can arise from the boundary condition specified for the atmosphere above the inversion region. French, Elliot, \& Gierasch (1978) further developed the inversion method by propagating errors from occultation light curves (both from the boundary condition fit and the fluxes used for the inversion) into the inversion profiles. They also expressed the pressure profile directly as an integral of the appropriate kernel over the refraction angle, rather than calculating the pressure from the number density profile as had been done in the past. Other applications of the inversion method to stellar occultation data have been presented by Roques et al. (1994) and Hubbard et al. (1990a, 1997).

The inversion of occultation light curves for smallbody atmospheres requires that we avoid certain assumptions that have been made for large bodies. In particular, we must relax the assumption that the local scale height is much less than the body's radius. Furthermore, the inversion for a small-body atmosphere must account for the change in local gravity by the inverse square of the radius and the lateral focusing of the refracted starlight by the curvature of the body's limb. These three effects are included in the inversion method presented in this paper. We also include the errors in the inversion results that arise from the random noise in the occultation light curve and consider the uncertainties caused by systematic errors in the zero stellar flux level. Our method allows an arbitrary boundary condition for the atmosphere that can be specified numerically or analytically. We illustrate how the boundary condition is used in our method by implementing, as an example, the small-body occultation light-curve model of Elliot \& Young (Paper I).

As two examples for application of this method, we invert the light curves for the 1988 June 9 occultation by Pluto observed with the KAO (Elliot et al. 1989; Paper I) and the 1997 November 4 occultation by Triton observed with the Fine Guidance Sensor (FGS) on the Hubble Space Telescope (HST; Elliot et al. 1998, 2000b). Inversions yield valid temperature profiles for these two bodies under the assumption that the structures of their occultation light curves are not significantly affected by extinction in their atmospheres - an assumption that is likely valid for Triton but may not be for Pluto. These results establish the atmospheric structures for the two bodies at the microbar level at the times of their respective occultations, and they can be compared with results of subsequent events when they become available - for example, the Triton occultation observed in 2001 (Person et al. 2001) and the Pluto occultations observed in 2002 (Buie et al. 2002; Elliot et al 2003; Sicardy et al. 2003).

\section{INVERSION OF AN OCCULTATION LIGHT CURVE}

In this section, we shall develop the equations needed for inversion of a stellar occultation light curve for a small body, in which the atmospheric scale height is not small rel- ative to the radius of the body. We first list our assumptions and derive the inversion equations for refractivity and pressure. Then, in $\S 3$, we cast these equations into summation form (suitable for numerical evaluation), followed by a derivation of the errors in $\S 4$ that propagate into the radius scale, temperature, pressure, and number density profiles from noise in the occultation light curve.

A stellar occultation by a small-body atmosphere is illustrated in Figure 1, where we see starlight impinging from the left. The approximately exponential density gradient in the atmosphere causes the starlight to be refracted toward the refractivity gradient, which lies in the radial direction for a spherically symmetric atmosphere.

\subsection{Assumptions}

We make the following assumptions about the occultations and the properties of the occulting body's atmosphere.

\subsubsection{Starlight}

1. The starlight impinging on the atmosphere comes from a monochromatic, point source.

\subsubsection{Occulting Atmosphere}

2. The only external force on the atmosphere arises from a point mass at its center, which produces a spherically symmetric gravity field that varies inversely as the radius squared.

3. The atmosphere is quiescent, so there are no forces arising from rotation or other motions.

4. The atmosphere has homogeneous composition, which implies that its refractivity (at standard temperature and pressure) and its molecular weight do not vary over the inversion region.

From the last three assumptions, it follows that the atmosphere is in hydrostatic equilibrium and that the atmospheric structure has spherical symmetry (i.e., refractivity, number density, pressure, temperature, and scale height are functions of radius only).

5. The mass of the occulting body's atmosphere is much smaller than the mass of the body.

6. There is no significant extinction within the inversion region of the atmosphere.

7. The atmospheric refractivity is much less than 1 within the inversion region.

\subsubsection{Geometry and Optics}

8. Light propagates through the atmosphere in accordance with the laws of geometric optics.

9. The refracted path of a ray within the atmosphere does not differ significantly from its undeflected path.

10. Light rays arrive at the observer in the same order in which they entered the atmosphere (i.e., no "ray crossing" occurs).

11. Refracted light from only one point on the limb is received by the observer.

12. Refraction angles are small enough for small-angle trigonometric approximations to be valid.

13. Curvature of the atmospheric limb in the plane perpendicular to the incoming starlight causes the refracted starlight to be concentrated in inverse proportion to the 


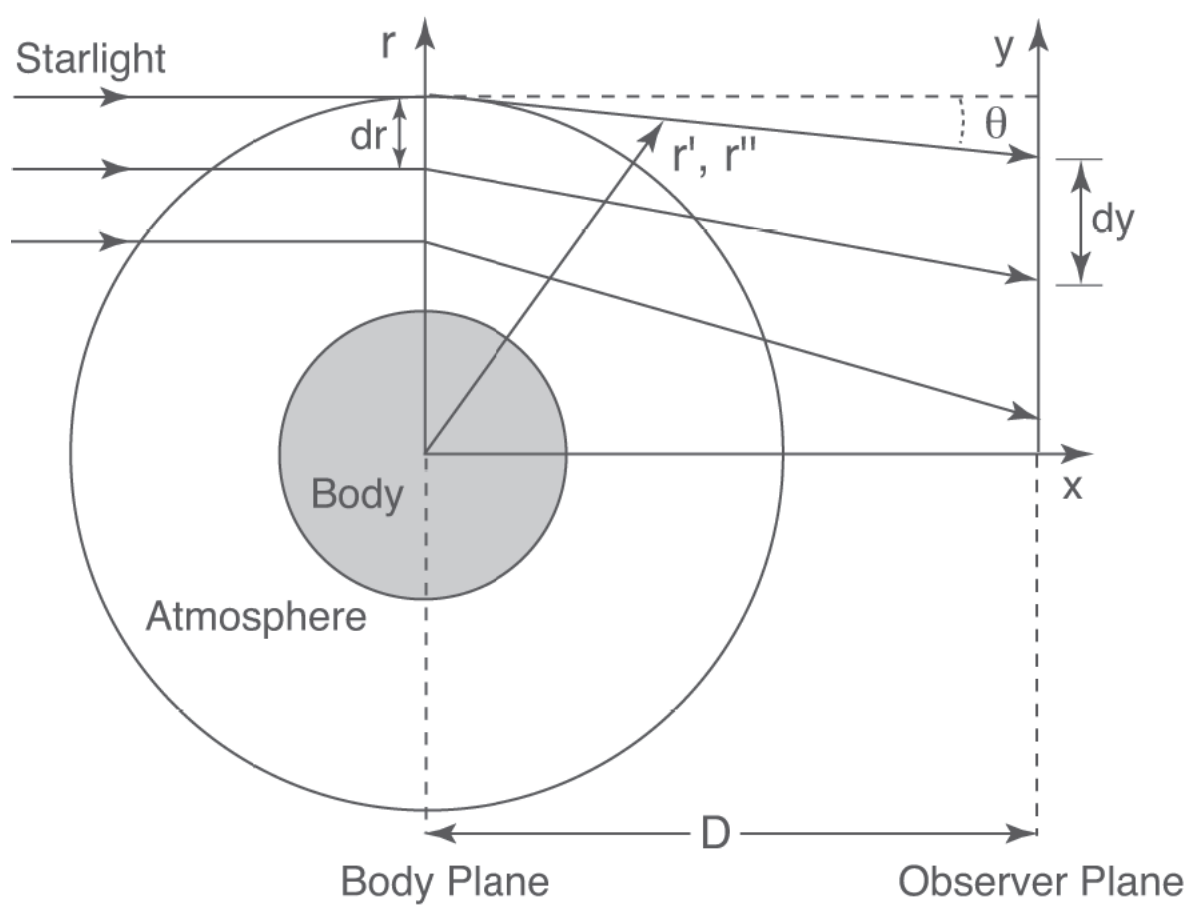

FIG. 1.-Refraction by a small-body atmosphere. This schematic drawing shows the coordinates used for the inversion calculations, where the body plane is perpendicular to the incident rays of starlight and passes through the center of the body. The $r$-axis lies in the body plane, with an origin at the center of the body. Rays of starlight entering from the left are refracted by the body's atmosphere by an angle $\theta$ as they travel to the right side of the diagram, the observer plane, which contains the $y$-axis (parallel to the $r$-axis). The $x$-axis has its origin at the center of the body and lies parallel to the unrefracted rays of starlight, $D$ is the distance between the two planes, and $r^{\prime}$ and $r^{\prime \prime}$ serve as variables of integration. In the observer plane, the starlight intensity is diminished by the ratio $|d r / d y|$ but is enhanced by a lateral focusing factor.

radial distance from the center of the body's shadow in the observer plane (Fig. 1).

\subsection{Radius Scale within the Atmosphere}

Our first task is to establish the minimum radius $r$ (within the atmosphere) experienced by a light ray arriving at a distance $y$ from the center of the body's stellar shadow at a distance $D$ from the body. Note in Figure 1 that $y$ can be negative if the ray is refracted past the center of the shadow. If $\theta(r)$ is the angle through which the ray is refracted (as defined by Fig. 1), these quantities are related by the equation

$$
\theta(r)=(y-r) / D \text {. }
$$

We can determine $r$ as a function of $y$ by using the stellar flux, $\phi(t)$. This flux has been corrected for background and normalized so that it equals 1.0 for the unocculted star and 0.0 when the star is fully occulted. From the geometric solution for the occultation observations (e.g., Elliot et al. 1993) or from geometric assumptions, we can determine the shadow radius as a function of time, $y(t)$, for the location of the observer. Using $y(t)$, we then express the stellar flux as a function of radius within the shadow: $\phi(y)$. We define another version of the stellar flux, $\phi_{\text {cyl }}(y)$, as the stellar flux that would have been recorded if the atmosphere were cylindrical, rather than spherical. The two fluxes, $\phi(y)$ and $\phi_{\text {cyl }}(y)$, differ only by the focusing effect of limb curvature (in the plane perpendicular to the impinging starlight), and they are related by the following equation, where we have substituted $|y|$ for the $\rho$ used in Paper I:

$$
\phi_{\text {cyl }}(y)=\frac{|y|}{r} \phi(y) \text {. }
$$

Following Paper I, we can write an expression for $\phi_{\text {cyl }}(y)$ in terms of the differentials $d r$ and $d y$ :

$$
\phi_{\mathrm{cyl}}(y)=\frac{d r}{d y} .
$$

We then combine equations (2) and (3) and integrate the result for $y>0$. Noting in the limit $r \rightarrow \infty$ that $\phi(y) \rightarrow 1$ and $r \rightarrow y$, we find

$$
r^{2}-y^{2}=2 \int_{y}^{\infty}\left[1-\phi\left(y^{\prime}\right)\right] y^{\prime} d y^{\prime},
$$

where $y^{\prime}$ is a variable of integration. We then solve for $r$ :

$$
r=\left\{y^{2}+2 \int_{y}^{\infty}\left[1-\phi\left(y^{\prime}\right)\right] y^{\prime} d y^{\prime}\right\}^{1 / 2} .
$$

Equation (5), combined with a knowledge of $\phi(y)$, allows us to compute $r$ as a function of $y$ and hence find $\theta(r)$ through equation (1), which will be needed in the inversion calculations described next.

\subsection{Refractivity and Number Density}

In deriving the refractivity of the atmosphere, we avoid the approximation that the scale height of the atmosphere is much less than its radius, as has been used in the past (e.g., 
Wasserman \& Veverka 1973). Our notation is defined in Figure 1. A ray proceeds along the $x$-axis, and as mentioned before, we assume that the deflection of the ray from this path within the atmosphere is negligible. A ray impinging on the atmosphere at a radius $r$ from the $x$-axis is refracted by an angle $\theta(r)$. This angle is defined to be positive above the $x$-axis, so that the atmospheric refraction depicted in Figure 1 produces negative refraction angles.

Denoting the index of refraction of the atmosphere by $N(r)$ and using equation (14) in $\S 3.2$ of Born \& Wolf (1964), we can express the refraction angle with the equation

$$
\theta(r)=\int_{-\infty}^{\infty} \frac{r}{r^{\prime}} \frac{d}{d r^{\prime}} \ln N\left(r^{\prime}\right) d x .
$$

In equation (6), the factor $r / r^{\prime}$ is the sine of the angle between the ray path and the refractivity gradient. From the geometry of Figure 1, we can write

$$
x^{2}=r^{\prime 2}-r^{2} .
$$

Putting equation (7) into differential form, we find

$$
\begin{gathered}
d x=\frac{r^{\prime}}{\sqrt{r^{\prime 2}-r^{2}}} d r^{\prime}, \\
\theta(r)=2 r \int_{r}^{\infty} \frac{d \ln N\left(r^{\prime}\right)}{d r^{\prime}} \frac{d r^{\prime}}{\sqrt{r^{\prime 2}-r^{\prime 2}}} .
\end{gathered}
$$

Our development now follows that used by Fjeldbo, Kliore, \& Eshleman (1971, hereafter FKE71) between their equations $(\mathrm{C} 10)$ and $(\mathrm{C} 11)$, which involves the inversion of an integral equation that was first solved by Abel in 1826 and has subsequently been used extensively in other contexts (e.g., seismology; Aki \& Richards 1980). We multiply both sides of equation (9) by the kernel $\left(r^{\prime \prime 2}-r^{2}\right)^{-1 / 2}$ and then integrate:

$$
\begin{aligned}
\int_{r}^{\infty} \frac{\theta\left(r^{\prime \prime}\right) d r^{\prime \prime}}{\sqrt{r^{\prime \prime 2}-r^{2}}}= & \int_{r}^{\infty} \frac{2 r^{\prime \prime}}{\sqrt{r^{\prime 2}-r^{2}}} \\
& \times\left[\int_{r^{\prime \prime}}^{\infty} \frac{d \ln N\left(r^{\prime}\right)}{d r^{\prime}} \frac{d r^{\prime}}{\sqrt{r^{\prime 2}-r^{\prime \prime 2}}}\right] d r^{\prime \prime} .
\end{aligned}
$$

Reversing the order of integration and incorporating the appropriate change of limits, we can reduce the right-hand side to a simple expression involving the index of refraction:

$$
\begin{aligned}
\int_{r}^{\infty} & \frac{\theta\left(r^{\prime \prime}\right) d r^{\prime \prime}}{\sqrt{r^{\prime \prime 2}-r^{2}}} \\
& =\int_{r}^{\infty} \frac{d \ln N\left(r^{\prime}\right)}{d r^{\prime}}\left(\int_{r}^{r^{\prime}} \frac{2 r^{\prime \prime} d r^{\prime \prime}}{\sqrt{r^{\prime \prime 2}-r^{2}} \sqrt{r^{\prime 2}-r^{\prime \prime 2}}}\right) d r^{\prime} \\
& =\int_{r}^{\infty} \frac{d \ln N\left(r^{\prime}\right)}{d r^{\prime}} 2\left[\sin ^{-1} \frac{\sqrt{r^{\prime \prime 2}-r^{2}}}{\sqrt{r^{\prime 2}-r^{2}}}\right]_{r^{\prime \prime}=r}^{r^{\prime \prime}=r^{\prime}} d r^{\prime} \\
& =\pi \int_{r}^{\infty} \frac{d \ln N\left(r^{\prime}\right)}{d r^{\prime}} d r^{\prime}=-\pi \ln N(r) .
\end{aligned}
$$

We denote the refractivity of the atmosphere as a function of radius by $\nu(r)$, which is related to the atmospheric index of refraction, $N(r)$ :

$$
N(r)=1+\nu(r) .
$$

Invoking our assumption that $\nu(r) \ll 1$, we can rewrite equation (11) as

$$
\nu(r)=-\frac{1}{\pi} \int_{r}^{\infty} \frac{\theta\left(r^{\prime}\right)}{\sqrt{r^{\prime 2}-r^{2}}} d r^{\prime} .
$$

In order to eliminate the infinite (but integrable) integrand at $r^{\prime}=r$, we integrate equation (13) by parts, making the substitution $\ln \left|x+\left(x^{2}-1\right)^{1 / 2}\right|=\cosh ^{-1} x$ :

$$
\begin{aligned}
\nu(r)=-\frac{1}{\pi}[ & \left.\theta\left(r^{\prime}\right) \cosh ^{-1}\left(\frac{r^{\prime}}{r}\right)\right|_{r^{\prime}=r} ^{r^{\prime}=\infty} \\
& \left.\quad-\int_{\theta(r)}^{0} \cosh ^{-1}\left(\frac{r^{\prime}}{r}\right) d \theta\left(r^{\prime}\right)\right] .
\end{aligned}
$$

Since $\theta(\infty)=0$ and $\cosh ^{-1}(1)=0$, the first term inside the brackets on the right-hand side of equation (14) is zero, and we find the following equation for the refractivity:

$$
\nu(r)=-\frac{1}{\pi} \int_{0}^{\theta(r)} \cosh ^{-1}\left(\frac{r^{\prime}}{r}\right) d \theta\left(r^{\prime}\right) .
$$

One may prefer to express the refractivity as an integral over radius and the derivative of the refraction angle:

$$
\nu(r)=\frac{1}{\pi} \int_{r}^{\infty} \cosh ^{-1}\left(\frac{r^{\prime}}{r}\right) \frac{d \theta\left(r^{\prime}\right)}{d r^{\prime}} d r^{\prime} .
$$

We can compare our result with that of FKE71, who derived an equation for the refractivity in terms of the refraction angle for spacecraft radio transmissions. For this case they did not assume that the refracted path of the ray within the atmosphere was significantly altered from its original path. Hence, they work with two parameters describing the path of the ray: the asymptotic distance $a_{1}$ of the undeflected ray from the center of mass, and the minimum radius $r_{01}$ that the deflected ray passes from the center of mass. In our formulation, both these quantities would equal our minimum radius, $r$. Also, they denote the index of refraction by $\mu\left(r_{01}\right)$ and the refraction angle by $\alpha$. If we make these substitutions of notation and use the definition of inverse hyperbolic cosine that we gave earlier, we see that our equation (15) agrees with their equation (C11).

We obtain the number density, $n(r)$, by making one more assumption: We know the refractivity of the atmosphere at standard temperature and pressure (STP) as a function of $r$, $\nu_{\mathrm{STP}}(r)$. Then we have

$$
n(r)=L \frac{\nu(r)}{\nu_{\mathrm{STP}}(r)},
$$

where $L$ is Loschmidt's number. If we assume that the composition of the atmosphere is homogeneous, then $\nu_{\mathrm{STP}}(r)$ is a constant, and the above equation becomes

$$
n(r)=L \frac{\nu(r)}{\nu_{\mathrm{STP}}} .
$$

\subsection{Pressure}

In order to calculate the pressure, $p(r)$, we now invoke our assumption that the atmosphere is in hydrostatic equilibrium. We define the molecular weight as a function of radius as $\mu(r)$. The change in pressure, $d p(r)$, in a layer $d r$ is 
given by the hydrostatic equation:

$$
d p(r)=-m_{\mathrm{amu}} \mu(r) n(r) g(r) d r,
$$

where $m_{\mathrm{amu}}$ is the atomic mass unit. Substituting equation (18) for $n(r)$, noting that $p(r) \rightarrow 0$ as $r \rightarrow \infty$, and integrating both sides using $r^{\prime}$ as the variable of integration, we obtain

$$
p(r)=\int_{r}^{\infty} \frac{m_{\mathrm{amu}} L}{\nu_{\mathrm{STP}}} \mu\left(r^{\prime}\right) g\left(r^{\prime}\right) \nu\left(r^{\prime}\right) d r^{\prime},
$$

where the gravitational acceleration is given by

$$
g\left(r^{\prime}\right)=G M_{p} / r^{\prime 2}
$$

with $M_{p}$ the mass of the occulting body and $G$ the universal gravitational constant. Substituting the expression for $n(r)$ given in equation (13) into equation (20) and assuming that the mean molecular weight of the atmosphere is constant, $\mu=\mu(r)$, we obtain the following double integral for the pressure:

$$
p(r)=-\frac{\mu m_{\mathrm{amu}} L G M_{p}}{\pi \nu_{\mathrm{STP}}} \int_{r}^{\infty} \int_{r^{\prime}}^{\infty} \frac{\theta\left(r^{\prime \prime}\right)}{r^{\prime 2} \sqrt{r^{\prime 2}-r^{\prime 2}}} d r^{\prime \prime} d r^{\prime} .
$$

Reversing orders of integration, the limits change for the $r^{\prime}$ integral from $r \leq r^{\prime} \leq \infty$ to $r \leq r^{\prime} \leq r^{\prime \prime}$, and equation (22) becomes

$$
p(r)=-\frac{\mu m_{\mathrm{amu}} L G M_{p}}{\pi \nu_{\mathrm{STP}}} \int_{r}^{\infty} \int_{r}^{r^{\prime \prime}} \frac{\theta\left(r^{\prime \prime}\right)}{r^{\prime 2} \sqrt{r^{\prime 2}-r^{\prime 2}}} d r^{\prime} d r^{\prime \prime} .
$$

We find that the inner integral is given by

$$
\int_{r}^{r^{\prime \prime}} \frac{d r^{\prime}}{r^{\prime 2} \sqrt{r^{\prime 2}-r^{\prime 2}}}=-\left.\frac{\sqrt{r^{\prime \prime 2}-r^{\prime 2}}}{r^{\prime 2} r^{\prime}}\right|_{r^{\prime}=r} ^{r^{\prime}=r^{\prime \prime}}=\frac{\sqrt{r^{\prime \prime 2}-r^{2}}}{r^{\prime \prime 2} r} .
$$

Substituting the above value of the integral into equation (23), we find

$$
p(r)=-\frac{\mu m_{\mathrm{amu}} L G M_{p}}{r \pi \nu_{\mathrm{STP}}} \int_{r}^{\infty} \frac{\theta\left(r^{\prime \prime}\right) \sqrt{1-\left(r / r^{\prime \prime}\right)^{2}} d r^{\prime \prime}}{r^{\prime \prime}} .
$$

To simplify the notation, we substitute $\gamma=r^{\prime \prime} / r$. Then we rewrite the integral in equation (25) in terms of $\gamma$ and integrate by parts:

$$
\begin{aligned}
\int_{1}^{\infty} \theta(\gamma) \gamma^{-1} & \sqrt{1-\gamma^{-2}} d \gamma \\
= & \theta(\gamma)\left[\cosh ^{-1} \gamma-\sqrt{1-\gamma^{-2}}\right]_{\gamma=1}^{\gamma=\infty} \\
& -\int_{\theta(1)}^{0}\left(\cosh ^{-1} \gamma-\sqrt{1-\gamma^{-2}}\right) d \theta(\gamma) .
\end{aligned}
$$

The first term on the right-hand side is zero, since $\theta(\infty)=0$ and the function of $\gamma$ is 0 for $\gamma=1$. Substituting $r^{\prime} / r$ for $\gamma$ and incorporating equation (26) into equation (25), we now have the expression for the pressure:

$$
\begin{aligned}
p(r)= & -\frac{\mu m_{\mathrm{amu}} L G M_{p}}{r \pi \nu_{\mathrm{STP}}} \\
& \times \int_{0}^{\theta(r)}\left[\cosh ^{-1}\left(\frac{r^{\prime}}{r}\right)-\sqrt{1-\left(\frac{r}{r^{\prime}}\right)^{2}}\right] d \theta\left(r^{\prime}\right) .
\end{aligned}
$$

\subsection{Temperature and Scale Height}

In order to simplify the equations for temperature and scale height that follow, we define the refractivity integral, $I_{\nu}(r)$, of equation (15) as

$$
I_{\nu}(r)=-\frac{1}{\pi} \int_{0}^{\theta(r)} \cosh ^{-1}\left(\frac{r^{\prime}}{r}\right) d \theta\left(r^{\prime}\right) .
$$

We also define the pressure integral, $I_{p}(r)$, as the pressure given by equation (27) without the physical constants:

$$
I_{p}(r)=-\frac{1}{\pi r} \int_{0}^{\theta(r)}\left[\cosh ^{-1}\left(\frac{r^{\prime}}{r}\right)-\sqrt{1-\left(\frac{r}{r^{\prime}}\right)^{2}}\right] d \theta\left(r^{\prime}\right) .
$$

The temperature, $T(r)$, is the ratio of the pressure to the number density and is given by the equation

$$
T(r)=\frac{p(r)}{k n(r)}=\frac{\mu m_{\mathrm{amu}} G M_{p} I_{p}(r)}{k I_{\nu}(r)} .
$$

We define the local pressure scale height, $H(r)$, as

$$
H(r)=\frac{R T(r)}{\mu g(r)}=\frac{k T(r)}{\mu m_{\mathrm{amu}} g(r)}=\frac{r^{2} I_{p}(r)}{I_{\nu}(r)}
$$

(see Paper I), where $R$ is the ideal gas constant and $k$ is Boltzmann's constant. Note that the local scale height depends on knowledge of neither the body's mass nor its atmospheric composition. It does, however, depend on the assumption that the molecular weight does not vary with radius, or $\mu(r)$ would appear within the integral for the pressure and not cancel out in equation (31).

\section{EVALUATION OF THE INVERSION INTEGRALS}

In this section we relate the refraction angles and radiicontained in the formal inversion integrals of the previous section - to the stellar fluxes recorded during an occultation. We represent the data as a set of stellar fluxes $\phi_{i}$ that are average values over the times, $\Delta t_{i}$. We shall refer to this set of fluxes and times as the occultation light curve. The fluxes are assumed to be normalized on a scale ranging from 0.0 to 1.0 (representing the totally occulted stellar flux, $\phi=0.0$, to the unocculted stellar flux, $\phi=1.0$ ), and the midtime of each integration interval is denoted by $t_{i}$. Each stellar flux is associated with a random error (noise), sampled from a Gaussian distribution of standard deviation $\sigma\left(\phi_{i}\right)$, and errors in the fluxes are assumed to be uncorrelated. Strictly speaking, the errors in the fluxes will follow a Poisson distribution, but for the large number of photons associated with occultation data of sufficient signal-to-noise ratio to carry out an inversion calculation, a Gaussian distribution is a good approximation. From the geometric solution to the occultation (see, e.g., Elliot et al. 1993), we calculate the radius $y_{i} \equiv y\left(t_{i}\right)$ from the center of the atmospheric shadow within a plane containing the observer that is perpendicular to the direction of the star. If we have $i_{\max }$ integrated stellar fluxes, we can then represent this data set as follows:

$$
\left\{y_{i}, \phi_{i}, \sigma\left(\phi_{i}\right)\right\}_{i=1, \ldots, i_{\max }} .
$$

If the initial data set represented by equation (32) contains 
some negative fluxes due to noise, then the adjacent data integration intervals (Fig. 2) must be averaged until a data set containing only positive fluxes is created. Another reason for averaging the data set would be to reduce the computation time required for the inversion calculations. Note that an arbitrary number of adjacent points in the data set can be averaged, and that the number of adjacent points averaged need not be the same for the entire data set.

Referring to Figure 2, which depicts a spherically symmetric atmosphere, we define a set of shells that correspond to the path of the starlight for each stellar flux in the occultation data set. The uppermost shell corresponds to index $i=1$, and $i$ increases as we define shells deeper into the atmosphere. We shall find it necessary to know the shadow radii at the boundaries of the shells. Thus, we define

$$
y_{i+1 / 2}=\left(y_{i}+y_{i+1}\right) / 2, \quad \Delta y_{i}=y_{i+1 / 2}-y_{i-1 / 2} .
$$

According to this definition, we can calculate the shadow radius at a shell boundary referring to either the shell above or the shell below: $y_{i+1 / 2}=y_{(i+1)-1 / 2}$. After calculating the shadow radii, we no longer need the midtimes or integration intervals - we shall use just the stellar fluxes and their corresponding shadow radii at the middle of the shells and at the shell boundaries:

$$
\left\{y_{i}, \Delta y_{i}, \phi_{i}, \sigma\left(\phi_{i}\right)\right\}_{i=1, \ldots, i_{\max }} .
$$

According to our indexing convention for the atmospheric shells, we note that the shadow radii $y_{i}$ will be decreasing with increasing $i$ for both immersion and emersion events.

\subsection{Boundary Condition for the Atmosphere above the Inversion Region}

Since the inversion integrals of equations (28) and (29) require knowledge of the atmospheric structure above the inversion region out to an infinite radius, we need a way to specify this structure so that it can be used as a boundary condition for the inversion calculation. We must also specify the uncertainties that the boundary condition introduces into the inversion results. Since several approaches to this task can be used, in this section we shall specify only what information must be supplied by the boundary condition in order to carry out the inversion. Specific approaches for specifying the boundary condition will be discussed later.

We allow that some of the data corresponding to the uppermost shells in the atmosphere may be used to specify the boundary condition, and these data would not be used in the inversion calculation. For this case, the inversion would begin at a lower shell with index $i_{b}\left(1 \leq i_{b}<i_{\max }\right)$. We denote the shadow radius at the top of this shell by $y_{b}$, which corresponds to the arrival radius of a light ray that had a closest-approach radius $r_{b}$ within the body's atmosphere. In addition to supplying these quantities, the boundary condition should also supply a standard deviation for $r_{b}$, which we denote $\sigma\left(r_{b}\right)$. Finally, the boundary condition should supply a function $\theta_{b}(r)$ that specifies the refraction angle for a light ray with a closest-approach radius $r$ and is defined for $r \geq r_{b}$.

With knowledge of $\theta_{b}(r)$, from which we can calculate the differential $d \theta_{b}(r)$, we define the refractivity boundary

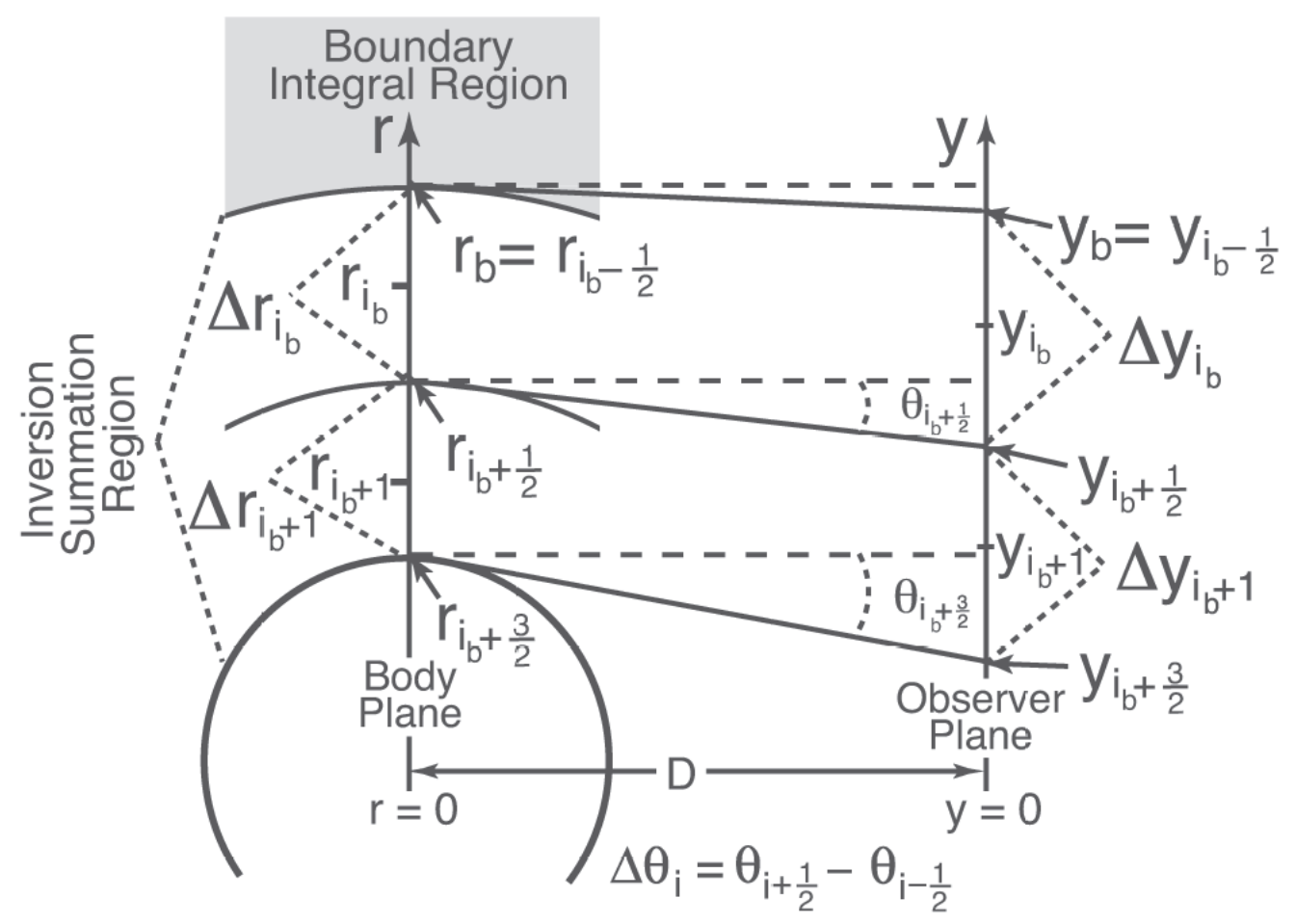

FIG. 2.- Shells used in the inversion summations. This magnified view of the body section of Fig. 1 shows our indexing scheme for the inversion shells. Note

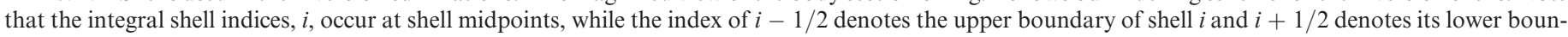

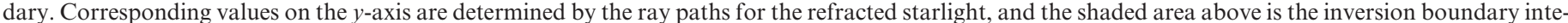
gral region. The first shell of the inversion region ("boundary shell ") begins at the boundary radius, $r=r_{b}$, and has an index $i=i_{b}$. 
integral, $B_{\nu}\left(r_{b}, r\right)$, as follows:

$$
B_{\nu}\left(r_{b}, r\right) \equiv-\frac{1}{\pi} \int_{0}^{\theta\left(r_{b}\right)} \cosh ^{-1}\left(\frac{r^{\prime}}{r}\right) d \theta_{b}\left(r^{\prime}\right), \quad r_{b} \geq r .
$$

We also define a pressure boundary integral, $B_{p}\left(r_{b}, r\right)$ :

$$
\begin{aligned}
& B_{p}\left(r_{b}, r\right) \\
& \equiv-\frac{1}{\pi r} \int_{0}^{\theta\left(r_{b}\right)}\left[\cosh ^{-1}\left(\frac{r^{\prime}}{r}\right)-\sqrt{1-\left(\frac{r}{r^{\prime}}\right)^{2}}\right] d \theta_{b}\left(r^{\prime}\right), \\
& r_{b} \geq r
\end{aligned}
$$

The boundary condition may also specify standard deviations for refractivity and pressure boundary integrals, which we denote $\sigma\left(B_{\nu}\left(r_{b}, r\right)\right)$ and $\sigma\left(B_{p}\left(r_{b}, r\right)\right)$, respectively.

Next we define the refractivity integral, $I_{\nu}\left(r_{b}, r\right)$, and the pressure integral, $I_{p}\left(r_{b}, r\right)$, over the region of atmosphere below $r_{b}$ :

$$
\begin{aligned}
& I_{\nu}\left(r_{b}, r\right) \equiv-\frac{1}{\pi} \int_{\theta\left(r_{b}\right)}^{\theta(r)} \cosh ^{-1}\left(\frac{r^{\prime}}{r}\right) d \theta\left(r^{\prime}\right), \quad r_{b} \geq r, \quad \\
& I_{p}\left(r_{b}, r\right) \\
& \equiv-\frac{1}{\pi r} \int_{\theta\left(r_{b}\right)}^{\theta(r)}\left[\cosh ^{-1}\left(\frac{r^{\prime}}{r}\right)-\sqrt{1-\left(\frac{r}{r^{\prime}}\right)^{2}}\right] d \theta\left(r^{\prime}\right) \\
& r_{b} \geq r .
\end{aligned}
$$

With these definitions, we can rewrite the inversion integrals given by equations (28) and (29) as sums over the boundary region and the inversion region:

$$
\begin{aligned}
& I_{\nu}(r)=B_{\nu}\left(r_{b}, r\right)+I_{\nu}\left(r_{b}, r\right), \\
& I_{p}(r)=B_{p}\left(r_{b}, r\right)+I_{p}\left(r_{b}, r\right) .
\end{aligned}
$$

\subsection{Summation Equations for the Inversion Integrals}

In this section we want to evaluate the inversion integrals, as given by equations (39) and (40). This requires us to relate the observed stellar occultation fluxes to the radii and refraction angles that appear in their integrands and to approximate the integrals as sums over the atmospheric shells (Fig. 2). To begin this task, we rewrite equation (5) as the sum of two integrals, one over the region of the atmosphere covered by the boundary condition and the other over the region of the atmosphere covered by the inversion. We also reverse the limits of integration to match the order of the anticipated summation, from higher to lower radii:

$r=\left\{y^{2}-2 \int_{y_{b}}^{y}\left[1-\phi\left(y^{\prime}\right)\right] y^{\prime} d y^{\prime}-2 \int_{\infty}^{y_{b}}\left[1-\phi\left(y^{\prime}\right)\right] y^{\prime} d y^{\prime}\right\}^{1 / 2}$.

Next we note that the radius for the boundary of the inversion, $r_{b}$, is given by the equation

$$
r_{b}^{2}=y_{b}^{2}-2 \int_{\infty}^{y_{b}}\left[1-\phi\left(y^{\prime}\right)\right] y^{\prime} d y^{\prime} .
$$

Substituting equation (42) into equation (41) and integrating the constant term, we find the following expression for $r$ :

$$
r=\left[r_{b}^{2}+2 \int_{y_{b}}^{y} \phi\left(y^{\prime}\right) y^{\prime} d y^{\prime}\right]^{1 / 2}
$$

In order to evaluate equation (43) as a sum over fluxes and shadow radii, we first write the sum that approximates the integral of equation (43) for calculating the body radius at the shell boundaries $r_{i+1 / 2}\left(\right.$ for $\left.i \geq i_{b}\right)$ :

$$
r_{i+1 / 2}=\left(r_{b}^{2}+2 \sum_{j=i_{b}}^{i} \phi_{j} y_{j} \Delta y_{j}\right)^{1 / 2}
$$

We can now define the radius increments for the $i$ th shell, $\Delta r_{i}$, as the differences between their values at the shell boundaries (for $i \geq i_{b}$ ):

$$
\Delta r_{i}=r_{i+1 / 2}-r_{i-1 / 2}
$$

The radius for the middle of each shell is defined as the average of the radii at the shell boundaries:

$$
r_{i}= \begin{cases}\left(r_{i+1 / 2}+r_{i-1 / 2}\right) / 2, & \text { if } i>i_{b} \\ \left(r_{i+1 / 2}+r_{b}\right) / 2, & \text { if } i=i_{b}\end{cases}
$$

Approximating the distance $D$ between the observer and the occulting body as being constant, we can write an expression for the refraction angle at the shell boundaries, $\theta_{i \pm 1 / 2}$, as a function of the observer-plane and sky-plane radii, $y_{i \pm 1 / 2}$ and $r_{i \pm 1 / 2}$ :

$$
\theta_{i \pm 1 / 2}=\left(y_{i \pm 1 / 2}-r_{i \pm 1 / 2}\right) / D \text {. }
$$

We define $\Delta \theta_{i}$ for the $i$ th shell as the difference between the refraction angle at the shell boundaries:

$$
\Delta \theta_{i} \equiv \theta_{i+1 / 2}-\theta_{i-1 / 2}=\left(\Delta y_{i}-\Delta r_{i}\right) / D .
$$

Before writing equations approximating the inversion sums, we note that if the data represented by equation (32) are averaged in equal time intervals, then as the stellar flux steadily decreases, the radii within the atmosphere found from equation (44) will become closer and closer together. If one wishes to maintain an approximately equal spacing for the inversion results at some shell thickness $\Delta r$ (Fig. 2) within the atmosphere of the occulting body, one can then choose a subset of the $r_{i+1 / 2}$ for which consecutive values differ by an amount as close to the desired spacing as possible. Corresponding values for $y_{i}, \Delta y_{i}, \phi_{i}, \sigma\left(\phi_{i}\right), \theta_{i}, \Delta \theta_{i}$, and $r_{i}$ are also obtained for later use. In the equations that follow, we shall use the index $i$ to refer to a contiguous set of $r_{i+1 / 2}$, $r_{i}$, and $\Delta \theta_{i}$-whether they be the original set or a subset that has been reindexed so that the $i$ represents a continuous set of integers.

We now write an equation for the sums that approximate the part of the inversion integrals (eqs. [37] and [38]) that include the inversion region, which, in the limit of small increments of refraction angle, would converge to the desired integrals:

$$
I_{\nu}\left(r_{b}, r\right) \approx-\frac{1}{\pi} \sum_{j=i_{b}}^{i} \cosh ^{-1}\left(\frac{r_{j}}{r_{i+1 / 2}}\right) \Delta \theta_{j}
$$




$$
\begin{aligned}
I_{p}\left(r_{b}, r\right) \approx-\frac{1}{\pi r_{i+1 / 2}} \sum_{j=i_{b}}^{i} & {\left[\cosh ^{-1}\left(\frac{r_{j}}{r_{i+1 / 2}}\right)\right.} \\
& \left.-\sqrt{1-\left(\frac{r_{i+1 / 2}}{r_{j}}\right)^{2}}\right] \Delta \theta_{j} .
\end{aligned}
$$

However, real data cannot be subdivided more finely than the original integration intervals, and we want the approximation to the integrals to converge as well as possible for the integration interval available. Hence, we follow the procedure of French et al. (1978) and integrate the functions of $r$ in equations (49) and (50) before summing, since these functions change quickly near $r_{j} / r_{i+1 / 2}=1$. We define the ratios

$$
z_{j-} \equiv \frac{r_{j-1 / 2}}{r_{i+1 / 2}}, \quad z_{j+} \equiv \frac{r_{j+1 / 2}}{r_{i+1 / 2}} .
$$

Then we integrate the functions of $r$ in equations (49) and (50) and define the sums $S_{\nu}\left(i_{b}, r_{i+1 / 2}\right)$ and $S_{p}\left(i_{b}, r_{i+1 / 2}\right)$, which will approximate the inversion integrals somewhat better than those indicated in equations (49) and (50) for equal-sized inversion shells (Figs. 1 and 2):

$$
\begin{aligned}
S_{\nu}\left(r_{b}, r_{i+1 / 2}\right) \equiv-\frac{1}{\pi} \sum_{j=i_{b}}^{i} & \frac{1}{z_{j+}-z_{j-}} \\
& \times\left[z \cosh ^{-1} z-\sqrt{z^{2}-1}\right]_{z_{j-}}^{z_{j+}} \Delta \theta_{j},
\end{aligned}
$$

$$
\begin{aligned}
S_{p}\left(r_{b}, r_{i+1 / 2}\right) \equiv- & \frac{1}{\pi} \sum_{j=i_{b}}^{i} \frac{1}{z_{j+}-z_{j-}} \\
& \times\left[z \cosh ^{-1} z-2 \sqrt{z^{2}-1}-\sin ^{-1} z\right]_{z_{j-}}^{z_{j+}} \Delta \theta_{j} .
\end{aligned}
$$

The equations for the refractivity, number density, pressure, temperature, and scale height at the lower boundary of each atmospheric shell within the inversion region can now be written in terms of the boundary integrals given by equations (35) and (36) and the summations given by equations (52) and (53):

$$
\begin{aligned}
\nu_{i+1 / 2} & \equiv \nu\left(r_{b}, r_{i+1 / 2}\right)=B_{\nu}\left(r_{b}, r_{i+1 / 2}\right)+S_{\nu}\left(i_{b}, r_{i+1 / 2}\right), \\
n_{i+1 / 2} & \equiv n\left(r_{b}, r_{i+1 / 2}\right)=\frac{L}{\nu_{\mathrm{STP}}} \nu\left(r_{b}, r_{i+1 / 2}\right), \\
p_{i+1 / 2} & \equiv p\left(r_{b}, r_{i+1 / 2}\right) \\
& =\frac{\mu m_{\mathrm{amu}} L G M_{p}}{\nu_{\mathrm{STP}}}\left[B_{p}\left(r_{b}, r_{i+1 / 2}\right)+S_{p}\left(i_{b}, r_{i+1 / 2}\right)\right], \\
T_{i+1 / 2} & \equiv T\left(r_{b}, r_{i+1 / 2}\right)=\frac{p\left(r_{b}, r_{i+1 / 2}\right)}{k n\left(r_{b}, r_{i+1 / 2}\right)} \\
& =\frac{\mu m_{\mathrm{amu}} G M_{p}\left[B_{p}\left(r_{b}, r_{i+1 / 2}\right)+S_{p}\left(i_{b}, r_{i+1 / 2}\right)\right]}{k\left[B_{\nu}\left(r_{b}, r_{i+1 / 2}\right)+S_{\nu}\left(i_{b}, r_{i+1 / 2}\right)\right]} \\
H_{i+1 / 2} & \equiv H\left(r_{b}, r_{i+1 / 2}\right) \\
& =\frac{r_{i+1 / 2}^{2}\left[B_{p}\left(r_{b}, r_{i+1 / 2}\right)+S_{p}\left(i_{b}, r_{i+1 / 2}\right)\right]}{B_{\nu}\left(r_{b}, r_{i+1 / 2}\right)+S_{\nu}\left(i_{b}, r_{i+1 / 2}\right)} .
\end{aligned}
$$

\subsection{A Specific Boundary Condition}

As discussed earlier, the boundary condition for the inversion must specify a boundary radius $r_{b}$ at which the inversion summations begin. It also must specify the refraction angle $\theta_{b}(r)$ and its differential $d \theta_{b}(r)$ for radii greater than $r_{b}$. Although there is some latitude in establishing the boundary condition, the value of $r_{b}$ and these functions are not completely arbitrary, in that they should reproduce the light curve corresponding to radii above $r_{b}$ as closely as possible. For example, the chosen value of $r_{b}$ should satisfy equation (5) both for the actual light curve and the synthetic light curve generated from the functions $\theta_{b}(r)$ and $d \theta_{b}(r)$ supplied by the boundary condition. Also, any additional parameters of the boundary condition should be adjusted so that the synthetic light curve generated from the boundary condition matches the actual light curve as closely as possible.

One approach for achieving a close relationship between the boundary condition and the actual light curve corresponding to radii greater than $r_{b}$ is to first fit a model to this portion of the light curve and then use the fitted model to determine $r_{b}$ and the refraction-angle function and its differential function. One such model, appropriate for Pluto and Triton, is that of Paper I, which allows a possible thermal gradient in the atmosphere. Paper I used a power-law dependence of temperature with radius, not for physical reasons but because this functional form allows a thermal gradient with fewer mathematical difficulties in generating a synthetic light curve than other functional forms considered. If we specify the half-light radius $r_{h}$ as the reference radius and set the temperature here as $T_{h}$, then the temperature as a function of radius, $T(r)$, is determined by a power index $b$ :

$$
T(r)=T_{h}\left(r / r_{h}\right)^{b}
$$

(Paper I). In Paper I, the half-light radius is defined as the radius within the body's atmosphere that corresponds to a normalized stellar flux of 0.5 in the observer plane, including the effects of differential refraction and lateral focusing (but not extinction).

The model in Paper I also allows a power-law dependence of the mean molecular weight as a function of radius, but we shall not use that generality here. Hence we set the powerlaw index $a$ for molecular weight variation equal to zero when using the equations in Paper I.

The temperature at half-light is most easily related to the light curve through an intermediate parameter $\lambda_{h}$ that is the ratio of gravitational potential energy to $k T_{h}$. The value of $T_{h}$ can be determined from $\lambda_{h}, r_{h}$, and other physical constants:

$$
T_{h}=\frac{\mu m_{\mathrm{amu}} G M_{p}}{k \lambda_{h} r_{h}}
$$

(Paper I). For a bound atmosphere, $\lambda_{h} \gg 1$ (e.g., $\lambda_{h} \sim 3000$ for Jupiter, $\sim 60$ for Triton, and $\sim 20$ for Pluto). Also, as discussed in Paper I, $\lambda_{h}=r_{h} / H_{h}$, where $H_{h}$ is the pressure scale height at half-light, and $\lambda(r)=\lambda_{h}\left(r / r_{h}\right)^{-(1+b)}$.

We can now write an equation for $\lambda(r)$, using its definition and equation (59):

$$
\lambda(r) \equiv \frac{\mu m_{\mathrm{amu}} G M_{p}}{r k T(r)}=\lambda_{h}\left(\frac{r}{r_{h}}\right)^{-(1+b)} .
$$


According to the fitting procedure recommended in Paper I, the "equivalent isothermal" lambda, $\lambda_{\text {hi }}$, is fit for instead of $\lambda_{h}$ because it is less correlated with the power index $b$. The two energy ratios are related by equation (5.13) in Paper I, which we repeat here (setting $a=0)$ :

$$
\lambda_{h}=\lambda_{\mathrm{hi}}-5 b / 2 .
$$

Hence, as the result of fitting the appropriate portion of a light curve to establish the boundary condition, we determine the parameters $\lambda_{h}, r_{h}$, and $b$ that describe the atmosphere within the boundary region.

We now use these parameters to find the required functions for the refraction angle, its derivative, and the boundary radius for the inversion, $r_{b}$. First we write an equation for the refraction angle within the boundary, $\theta_{b}(r)$ (given by eq. [4.6] of Paper I). We use a slightly different notation from that of Paper I to emphasize the $r$-dependence:

$$
\theta_{b}(r)=-\sqrt{2 \pi \lambda(r)} \nu(r) A(r, b) .
$$

In equation (63), the function $A(r, b)$ is an asymptotic power series in the parameters $b$ and $r$ [found by setting the parameter $a=0$ in eq. (A2) in Paper I, which has an erroneous coefficient of the $b^{3} \lambda^{-4}(r)$ term that has been corrected here]:

$$
\begin{aligned}
A(r, b)= & 1-\frac{3-3 b}{8} \lambda^{-1}(r)-\frac{15-14 b-b^{2}}{128} \lambda^{-2}(r) \\
& -\frac{105-27 b-69 b^{2}-9 b^{3}}{1024} \lambda^{-3}(r) \\
& -\frac{4725+4236 b-4706 b^{2}-3764 b^{3}-491 b^{4}}{32768} \lambda^{-4}(r) \\
& +\cdots .
\end{aligned}
$$

The refractivity, $\nu(r)$, is given in terms of the refractivity at half-light $\nu_{h}$ and previously defined quantities:

$$
\nu(r)=\nu_{h}\left(\frac{r}{r_{h}}\right)^{b} \exp \left[\frac{\lambda(r)-\lambda_{h}}{1+b}\right] .
$$

For $b=0$ and for the approximation of constant gravitational acceleration, the right-hand side of equation (65) reduces to the familiar form $\nu_{h} \exp \left[-\left(r-r_{h}\right) / H_{h}\right]$, where $H_{h}$ is the pressure scale height (Paper I). Equation (4.28) of Paper I gives an expression for $\nu_{h}$ :

$$
\begin{aligned}
\nu_{h}=\frac{r_{h}}{2 D \sqrt{2 \pi \lambda_{h}}}[ & 1-\frac{1}{\lambda_{h} B\left(r_{h}, b\right)} \\
& \left.-\sqrt{1-\frac{6}{\lambda_{h} B\left(r_{h}, b\right)}+\frac{1}{\lambda_{h}^{2} B\left(r_{h}, b\right)^{2}}}\right] .
\end{aligned}
$$

To evaluate equation (66), we need an expression for the asymptotic power series, $B(r, b)$, which can be found by evaluating equation (A5) in Paper I with the parameter $a$ set to zero. An erroneous coefficient of the $b^{2} \lambda^{-4}(r)$ term in Paper I has been corrected, and we use a slightly different notation to emphasize the $r$-dependence:

$$
\begin{aligned}
B(r, b)= & 1+\frac{1+15 b}{8} \lambda^{-1}(r)+\frac{9-34 b+25 b^{2}}{128} \lambda^{-2}(r) \\
& +\frac{75-81 b+b^{2}+5 b^{3}}{1024} \lambda^{-3}(r) \\
& +\frac{3675-1356 b-2110 b^{2}-268 b^{3}+59 b^{4}}{32768} \lambda^{-4}(r) \\
& +\cdots .
\end{aligned}
$$

Having all the information needed to calculate the refraction angle, we iteratively solve the equation below to find $r_{b}$ in terms of the fitted model parameters and $y_{b}$ :

$$
r_{b}=y_{b}-D \theta_{b}\left(r_{b}\right) .
$$

Finally, we write the equation for the differential of the refraction angle, as given by equation (4.9) of Paper I:

$$
d \theta_{b}(r)=\sqrt{2 \pi \lambda(r)^{3}} \frac{\nu(r)}{r} B(r, b) d r .
$$

This completes our specification of the boundary condition for the power-law thermal model of Paper I, which is summarized by equations (63), (68), and (69).

\section{EVALUATION OF ERRORS IN THE INVERSION RESULTS}

In this section we shall evaluate the random errors in the radius scale, refractivity, number density, pressure, temperature, and scale height that arise from the random errors in the fluxes, $\sigma\left(\phi_{i}\right)$. Errors arising from other causes will be discussed in $\S 7.4$. In the first subsection, $\S 4.1$, we consider the random errors that arise from the boundary condition and provide a general method for combining these with those from the inversion summations. Specific equations for applying this method to each of the atmospheric quantities of interest are given in subsequent subsections.

\subsection{Combining Errors from the Boundary Condition and Inversion Sums}

The parameters describing the boundary condition will introduce errors into the results of the inversion in a manner that will depend on how the boundary condition is specified. If we use the boundary condition specified in $\S 3.3$, which is a least-squares fit of a model to the portion of the light curve that lies outside the inversion sums, then the boundary contribution to the errors in the inversion is straightforward. One class of boundary conditions includes those with parameters determined from a least-squares fit to the portion of the light curve that corresponds to the atmosphere above the boundary. For this class, we assume that we have a function $f$ that can have arguments that do not have random errors (i.e., not included as fitted parameters). The function also has arguments $q_{1}, \ldots, q_{N}$ that are fitted (which we shall refer to as the boundary parameters), and the $j$ th and $k$ th parameters have a covariance, $\operatorname{Cov}\left(q_{j}, q_{k}\right)$. The covariances are determined by the least-squares fit (Bevington \& Robinson 1992). Alternatively, we can define the correlation coefficient, $\rho_{j k}$, in terms of the covariances 
and standard deviations, $\sigma\left(q_{j}\right)$ and $\sigma\left(q_{k}\right)$, of the boundary parameters with the following equation:

$$
\rho_{j k}=\frac{\operatorname{Cov}\left(q_{j}, q_{k}\right)}{\sigma\left(q_{j}\right) \sigma\left(q_{k}\right)} .
$$

The standard deviation of $f, \sigma(f)$, is then given by the equation

$$
\sigma(f)=\left[\sum_{j=1}^{N} \sum_{k=1}^{N} \frac{\partial f}{\partial q_{j}} \frac{\partial f}{\partial q_{k}} \rho_{j k} \sigma\left(q_{j}\right) \sigma\left(q_{k}\right)\right]^{1 / 2} .
$$

For the example in $\S 3.3$, the fitted parameters are $\lambda_{\text {hi }}, r_{h}$, and $b$. With knowledge of these, the quantities needed to calculate the boundary conditions for the inversion can be obtained, working backward from equations (63), (68), and (69).

We now note that all quantities determined from the inversion (including the boundary condition) can be calculated from the boundary parameters $q_{1}, \ldots, q_{N}$. These consolidate the relevant information contained in the fluxes $\left\{\varphi_{i}\right\}_{i=1, \ldots, i_{b}-1}$ that passed through the atmosphere above the boundary. As the fluxes remaining in the light curve (those passing through the atmosphere below the boundary, $\left.\left\{\varphi_{i}\right\}_{i=i_{b}, \ldots, i_{\max }}\right)$ were not used in establishing the boundary condition, they are uncorrelated with the boundary parameters, for we have already stipulated that the fluxes are uncorrelated with each other.

Knowing the error for each uncorrelated parameter, including the fluxes, we can calculate the error for any quantity associated with the $i$ th atmospheric shell, $x_{i}$ (or $x_{i+1 / 2}$ ), with the usual methods of error propagation (Bevington \& Robinson 1992). We define $\zeta\left(x_{i}\right)$ to be an operator that can be used to generate the standard deviation of the calculated quantity $x_{i}$. It is given by the equation

$$
\begin{gathered}
\zeta\left(x_{i}\right) \equiv\left[\sum_{j=1}^{N} \sum_{k=1}^{N} \frac{\partial x_{i}}{\partial q_{j}} \frac{\partial x_{i}}{\partial q_{k}} \rho_{j k} \sigma\left(q_{j}\right) \sigma\left(q_{k}\right)\right. \\
\left.+\sum_{k=i_{b}}^{i}\left(\frac{\partial x_{i}}{\partial \phi_{k}}\right)^{2} \sigma^{2}\left(\phi_{k}\right)\right]^{1 / 2} .
\end{gathered}
$$

We shall use equation (72) as the basis for calculating the error for the radius scale and all atmospheric quantities derived from the inversion.

\subsection{Errors in the Radius Scale}

To calculate the error in the radius scale, we begin with the first term on the right-hand side of equation (72), which corresponds to the error contribution from the region of the light curve included in the boundary condition. For this case, the information from the fitted parameters $\left(\lambda_{\mathrm{hi}}, r_{h}\right.$, and $b$ ) for our example boundary condition can be consolidated into a single parameter, $r_{b}$. Hence we can write equation (72) for the standard deviation of $r_{i+1 / 2}$ by using the definition of $r_{i+1 / 2}$ given by equation (44):

$$
\begin{aligned}
\sigma\left(r_{i+1 / 2}\right)=\zeta\left(r_{i+1 / 2}\right)= & {\left[\left(\frac{\partial r_{i+1 / 2}}{\partial r_{b}}\right)^{2} \sigma^{2}\left(r_{b}\right)\right.} \\
& \left.+\sum_{k=i_{b}}^{i}\left(\frac{\partial r_{i+1 / 2}}{\partial \phi_{k}}\right)^{2} \sigma^{2}\left(\phi_{k}\right)\right]^{1 / 2} .
\end{aligned}
$$

Again using equation (44), we determine the partial derivatives required by the right-hand side of equation (73):

$$
\begin{gathered}
\frac{\partial r_{i+1 / 2}}{\partial r_{b}}=\frac{r_{b}}{r_{i+1 / 2}}, \\
\frac{\partial r_{i+1 / 2}}{\partial \phi_{k}}= \begin{cases}y_{k} \Delta y_{k} / r_{i+1 / 2}, & \text { if } i_{b} \leq k \leq i, \\
0, & \text { otherwise } .\end{cases}
\end{gathered}
$$

Substituting these derivatives into equation (73), we find

$$
\sigma\left(r_{i+1 / 2}\right)=\frac{1}{r_{i+1 / 2}}\left[r_{b}^{2} \sigma^{2}\left(r_{b}\right)+\sum_{k=i_{b}}^{i} y_{k}^{2} \Delta y_{k}^{2} \sigma^{2}\left(\phi_{k}\right)\right]^{1 / 2} .
$$

In this result, we determine $r_{b}$ and $\sigma\left(r_{b}\right)$ from the boundary condition - using equation (71) - and the $y_{k}$ are found from the geometric solution for the occultation. The latter should have negligible errors.

We square equation (76) and define two quantities that we shall find useful later. We define $\sigma_{q, r}^{2}$ as the contribution to the variance in $r_{i+1 / 2}$ arising from the boundary condition and $\sigma_{\phi, r}^{2}$ as the contribution to the variance in $r_{i+1 / 2}$ arising from the flux summation terms in the inversion:

$$
\begin{gathered}
\sigma_{q, r}^{2} \equiv r_{b}^{2} \sigma^{2}\left(r_{b}\right) / r_{i+1 / 2}^{2}, \\
\sigma_{\phi, r}^{2} \equiv\left[\sum_{k=i_{b}}^{i} y_{k}^{2} \Delta y_{k}^{2} \sigma^{2}\left(\phi_{k}\right)\right] / r_{i+1 / 2}^{2} .
\end{gathered}
$$

Thus, equation (76) can be rewritten as follows:

$$
\sigma(r)=\sqrt{\sigma_{q, r}^{2}+\sigma_{\phi, r}^{2}} .
$$

\subsection{Errors in Refractivity and Number Density}

To generate an expression for the rms errors in the refractivity and number density, we apply equation (72) to equation (54). Our equations will be more compact here and in the following sections if we make the definitions $B_{\nu} \equiv B_{\nu}\left(r_{b}, r_{i+1 / 2}\right), \quad B_{p} \equiv B_{p}\left(r_{b}, r_{i+1 / 2}\right), \quad S_{\nu} \equiv S_{\nu}\left(r_{b}, r_{i+1 / 2}\right)$, and $S_{p} \equiv S_{p}\left(r_{b}, r_{i+1 / 2}\right)$. With this notation, we write the equation for the rms error in the refractivity as

$$
\sigma\left(\nu_{i+1 / 2}\right)=\zeta\left(B_{\nu}+S_{\nu}\right) .
$$

The error in the number density is proportional to the error in refractivity:

$$
\sigma\left(n_{i+1 / 2}\right)=\frac{L}{\nu_{\mathrm{STP}}} \sigma\left(\nu_{i+1 / 2}\right) .
$$

Evaluation of the boundary parameter term in equation (80) is a straightforward application of equation (71):

$$
\sigma_{q, \nu}^{2}=\sum_{j=1}^{N} \sum_{k=1}^{N}\left(\frac{\partial B_{\nu}}{\partial q_{j}}+\frac{\partial S_{\nu}}{\partial q_{j}}\right)\left(\frac{\partial B_{\nu}}{\partial q_{k}}+\frac{\partial S_{\nu}}{\partial q_{k}}\right) \rho_{j k} \sigma\left(q_{j}\right) \sigma\left(q_{k}\right) .
$$

To evaluate the summation part, we need the partial derivatives that appear in the second term on the right-hand side of equation (72), which we evaluate numerically to find the variance of $S_{\nu}$ :

$$
\sigma_{\phi, \nu}^{2}=\sum_{k=i_{b}}^{i}\left(\frac{\partial B_{\nu}}{\partial \phi_{k}}+\frac{\partial S_{\nu}}{\partial \phi_{k}}\right)^{2} \sigma^{2}\left(\phi_{k}\right)
$$


Finally, we write the final result for the rms error in the refractivity, which can be evaluated with equations (82) and (83):

$$
\sigma\left(\nu_{i+1 / 2}\right)=\sqrt{\sigma_{q, \nu}^{2}+\sigma_{\phi, \nu}^{2}} .
$$

\subsection{Errors in the Pressure}

Evaluation of the rms error in the pressure follows equations that are completely analogous to those for the refractivity, beginning with equation (80):

$$
\begin{gathered}
\sigma\left(p_{i+1 / 2}\right)=\frac{\mu m_{\mathrm{amu}} L G M_{p}}{\nu_{\mathrm{STP}}} \zeta\left(B_{p}+S_{p}\right) \\
\sigma_{q, p}^{2}=\sum_{j=1}^{N} \sum_{k=1}^{N}\left(\frac{\partial B_{p}}{\partial q_{j}}+\frac{\partial S_{p}}{\partial q_{j}}\right)\left(\frac{\partial B_{p}}{\partial q_{k}}+\frac{\partial S_{p}}{\partial q_{k}}\right) \rho_{j k} \sigma\left(p_{j}\right) \sigma\left(p_{k}\right) \\
\sigma_{\phi, p}^{2}=\sum_{k=i_{b}}^{i}\left(\frac{\partial B_{p}}{\partial \phi_{k}}+\frac{\partial S_{p}}{\partial \phi_{k}}\right)^{2} \sigma^{2}\left(\phi_{k}\right) \\
\sigma\left(p_{i+1 / 2}\right)=\sqrt{\sigma_{q, p}^{2}+\sigma_{\phi, p}^{2}}
\end{gathered}
$$

\subsection{Errors in the Temperature}

Equations for the random errors in the temperature are more complex than those for refractivity and pressure, since the temperature involves a ratio of quantities derived from random variables. We begin by writing the rms error for the temperature with equation (72):

$$
\begin{aligned}
\sigma\left(T_{i+1 / 2}\right)= & \frac{\mu m_{\mathrm{amu}} G M_{p}}{k} \\
& \times \zeta\left(\frac{B_{p}\left(r_{b}, r_{i+1 / 2}\right)+S_{p}\left(i_{b}, r_{i+1 / 2}\right)}{B_{\nu}\left(r_{b}, r_{i+1 / 2}\right)+S_{\nu}\left(i_{b}, r_{i+1 / 2}\right)}\right) .
\end{aligned}
$$

Next we continue the calculation by finding the contribution to the variance in the temperature that arises from the boundary parameters, $\sigma_{q, T}^{2}$, and the contribution to the variance in the temperature that arises from the errors in the fluxes, $\sigma_{\phi, T}^{2}$. First we write the expression for $\sigma_{q, T}^{2}$ by applying equation (71):

$$
\begin{aligned}
\sigma_{q, T}^{2}= & \frac{1}{\left(B_{\nu}+S_{\nu}\right)^{4}} \\
\times & \times \sum_{j=1}^{N} \sum_{k=1}^{N}\left[\left(B_{\nu}+S_{\nu}\right)\left(\frac{\partial B_{p}}{\partial q_{j}}+\frac{\partial S_{p}}{\partial q_{j}}\right)\right. \\
& \left.\quad-\left(B_{p}+S_{p}\right)\left(\frac{\partial B_{\nu}}{\partial q_{j}}+\frac{\partial S_{\nu}}{\partial q_{j}}\right)\right] \\
\times & \quad\left[\left(B_{\nu}+S_{\nu}\right)\left(\frac{\partial B_{p}}{\partial q_{k}}+\frac{\partial S_{p}}{\partial q_{k}}\right)\right. \\
& \left.\quad-\left(B_{p}+S_{p}\right)\left(\frac{\partial B_{\nu}}{\partial q_{k}}+\frac{\partial S_{\nu}}{\partial q_{k}}\right)\right] \rho_{j k} \sigma\left(q_{j}\right) \sigma\left(q_{k}\right) .
\end{aligned}
$$

We note that all quantities needed to evaluate equation (90) have appeared previously. Next we write an equation for $\sigma_{\phi}^{2}$, again noting that all quantities in the equation have appeared previously:

$$
\begin{aligned}
& \sigma_{\phi, T}^{2}= \frac{1}{\left(B_{\nu}+S_{\nu}\right)^{4}} \\
& \times \sum_{k=i_{b}}^{i}\left[\left(B_{\nu}+S_{\nu}\right)\left(\frac{\partial B_{p}}{\partial \phi_{k}}+\frac{\partial S_{p}}{\partial \phi_{k}}\right)\right. \\
&\left.\quad-\left(B_{p}+S_{p}\right)\left(\frac{\partial B_{\nu}}{\partial \phi_{k}}+\frac{\partial S_{\nu}}{\partial \phi_{k}}\right)\right]^{2} \sigma^{2}\left(\phi_{k}\right) .
\end{aligned}
$$

Finally, we write the equation for the desired result, which can be evaluated with the aid of equations (90) and (91):

$$
\sigma\left(T_{i+1 / 2}\right)=\frac{\mu m_{\mathrm{amu}} G M_{p}}{k} \sqrt{\sigma_{q, T}^{2}+\sigma_{\phi, T}^{2}} .
$$

\subsection{Errors in the Scale Height}

We derive an expression for the rms error in the scale height, again beginning with equation (72):

$$
\sigma\left(H_{i+1 / 2}\right)=\zeta\left(r_{i+1 / 2}^{2} \frac{B_{p}+S_{p}}{B_{\nu}+S_{\nu}}\right) .
$$

Here we have a situation similar to the calculation of the temperature error in the previous subsection, except for the added complication of the multiplicative factor $r_{i+1 / 2}^{2}$. We approach the calculation in the same way as we did for the temperature, first finding the contribution to the variance of the scale height, $\sigma^{2}\left(H_{i+1 / 2}\right)$, that arises from the boundary parameters, $\sigma_{q, H}^{2}$, and then finding the contribution to the variance that arises from the summation of the flux terms in the inversion, $\sigma_{\phi, H}^{2}$. In this case the error in $r_{i+1 / 2}$ has contributions from both the boundary parameters and the inversion fluxes, so it must be included in both the boundary and summation variances.

First we write the equation for the contribution to the variance arising from the boundary parameters:

$$
\begin{aligned}
\sigma_{q, H}^{2}= & \frac{1}{\left(B_{\nu}+S_{\nu}\right)^{4}} \\
\times \sum_{j=1}^{N} \sum_{k=1}^{N}\left\{\left(B_{\nu}+S_{\nu}\right)\right. & {\left[r_{i+1 / 2}^{2}\left(\frac{\partial B_{p}}{\partial q_{j}}+\frac{\partial S_{p}}{\partial q_{j}}\right)\right.} \\
& \left.+\left(B_{p}+S_{p}\right) \frac{\partial r_{i+1 / 2}^{2}}{\partial q_{j}}\right] \\
& \left.-r_{i+1 / 2}^{2}\left(B_{p}+S_{p}\right)\left(\frac{\partial B_{\nu}}{\partial q_{j}}+\frac{\partial S_{\nu}}{\partial q_{j}}\right)\right\} \\
& \times\left\{( B _ { \nu } + S _ { \nu } ) \left[r_{i+1 / 2}^{2}\left(\frac{\partial B_{p}}{\partial q_{k}}+\frac{\partial S_{p}}{\partial q_{k}}\right)\right.\right. \\
& \left.+\left(B_{p}+S_{p}\right) \frac{\partial r_{i+1 / 2}^{2}}{\partial q_{k}}\right] \\
& \left.-r_{i+1 / 2}^{2}\left(B_{p}+S_{p}\right)\left(\frac{\partial B_{\nu}}{\partial q_{k}}+\frac{\partial S_{\nu}}{\partial q_{k}}\right)\right\} \\
& \times \rho_{j k} \sigma\left(q_{j}\right) \sigma\left(q_{k}\right) .
\end{aligned}
$$

Next we write an equation for the contribution to the variance arising from the summation in fluxes, keeping the 
$r_{i+1 / 2}^{2}$ factor grouped with the pressure summation term:

$$
\begin{aligned}
\sigma_{\phi, H}^{2}= & \frac{1}{\left(B_{\nu}+S_{\nu}\right)^{4}} \\
& \times \sum_{k=i_{b}}^{i}\left\{( B _ { \nu } + S _ { \nu } ) \left[r_{i+1 / 2}^{2} \frac{\partial\left(B_{p}+S_{p}\right)}{\partial \phi_{k}}\right.\right. \\
& \left.+\left(B_{p}+S_{p}\right) \frac{\partial r_{i+1 / 2}^{2}}{\partial \phi_{k}}\right] \\
& \left.-r_{i+1 / 2}^{2}\left(B_{p}+S_{p}\right) \frac{\partial\left(B_{\nu}+S_{\nu}\right)}{\partial \phi_{k}}\right\}^{2} \sigma^{2}\left(\phi_{k}\right) .
\end{aligned}
$$

Two derivatives in equation (95) require evaluation:

$$
\begin{aligned}
\frac{\partial r_{i+1 / 2}^{2} S_{p}}{\partial \phi_{k}} & =r_{i+1 / 2}^{2} \frac{\partial S_{p}}{\partial \phi_{k}}+2 r_{i+1 / 2} S_{p} \frac{\partial r_{i+1 / 2}}{\partial \phi_{k}} \\
& =r_{i+1 / 2}^{2} \frac{\partial S_{p}}{\partial \phi_{k}}+2 y_{k} \Delta y_{k} S_{p}, \\
\frac{\partial r_{i+1 / 2}^{2}}{\partial \phi_{k}} & =2 r_{i+1 / 2} \frac{\partial r_{i+1 / 2}}{\partial \phi_{k}}=2 y_{k} \Delta y_{k},
\end{aligned}
$$

where the flux derivative for $r_{i+1 / 2}$ is given by equation (75). Finally, we write the desired error in scale height as the square root of the sum of the variances given by equations (94) and (95):

$$
\sigma\left(H_{i+1 / 2}\right)=\sqrt{\sigma_{q, H}^{2}+\sigma_{\phi, H}^{2}} .
$$

\section{NUMERICAL EVALUATION OF THE INVERSION INTEGRALS}

In order to provide a standard, trustworthy, and repeatable procedure for the calculations described by our equations, we designed and implemented a set of functions and a template using the program Mathematica 4.0 (Wolfram 1999). Each function implements equations or parts of equations from this paper. The template then uses these functions and the data provided by the user to perform the desired inversion calculations.

Numerical evaluation of the inversion requires inputs of various types. First, there are the physical parameters such as distance, mass, composition of the body's atmosphere, and the physical constants needed to calculate pressure, density, and temperature. Second, we need a set of parameters that specify the atmospheric structure within the boundary region. Third, we need the normalized stellar fluxes $\left\{\phi_{i}\right\}$, their errors $\left\{\sigma\left(\phi_{i}\right)\right\}$, and their corresponding coordinates in the observer plane $\left\{y_{i}\right\}$. Finally, two parameters for the inversion calculations must be provided: (1) the (minimum) shell thickness and (2) the position in the list of normalized stellar fluxes that specifies the transition from the boundary region to the inversion region (Fig. 2). Although the fluxes are normalized, some may still be less than zero because of noise in the data. Therefore, we average any negative flux with the flux or fluxes that follow until all the final averaged fluxes are positive. Mid-y's and flux errors are then generated to correspond to these averaged flux values. As mentioned in $\S 3$, we may choose to reduce the resolution of the data by averaging several flux, flux error, and mid- $y$ points. This and the procedure to obtain positive flux may be performed in either order. We have chosen to reduce the resolution first, because the reduction in data resolution may remove some negative flux values (or eliminate them entirely).

The calculations take place in several steps. First we perform a least-squares fit with the mid-y's, fluxes, and flux errors above the boundary to determine the boundary model parameters and their errors. Next we use these fitted parameters and other values to calculate the values of $y$, radius, and theta at the boundary $\left(y_{b}, r_{b}, \theta_{b}\right)$. Then we use the mid-y's, fluxes, flux errors below the boundary, and delta $y$ 's $\left(\Delta y_{i}\right)$ - calculated from equations (41) through (48) - to calculate the lower boundary radii, mid-radii, delta radii, lower boundary theta, and delta theta $\left(r_{i+1 / 2}, r_{i}, \Delta r, \theta_{i+1 / 2}, \Delta \theta\right)$. The next step is to combine the adjacent data as necessary so that the delta radii are (the minimum amount) larger then the specified minimum shell thickness. Finally, we use these data lists to calculate the refractivity, density, pressure, temperature, and scale height with equations (54) through (58) and their errors with equations (76) through (98). This calculation flow is diagrammed in Figure 3.

In calculating the errors, we found that - except for the radii-analytical derivatives are difficult to derive and implement. Therefore, those derivatives are calculated numerically (as one-sided finite differences, to save computation time). We wrote one set of generic numerical derivative and variance functions for the boundary terms and another set for the summation terms. These two sets of functions together correspond to equation (72). For the boundary terms, each fitted parameter is individually stepped by $1 / 10$ of its formal error, and the resulting derivatives are combined with the errors and the correlation matrix to return the variance. For the summation terms, we individually stepped each flux by a tenth of its error, and then we recalculated the lower boundary radii, mid-radii, delta theta, etc., from these stepped fluxes and the corresponding $y$ 's to find the variances. It is important to note the intermediate results that are calculated from the fitted boundary parameters and fluxes and to make sure that all these intermediate results are recalculated with the stepped values of the fluxes.

After calculating the atmospheric profiles and their errors, standard plots and tables are generated to display the results, and the template "notebook" writes these results to a file for future use.

\section{TESTS WITH SYNTHETIC LIGHT CURVES}

In order to test the reliability of our method, we carried out inversions on a set of synthetic light curves. Our objective was to examine how the inversion temperature profiles changed for model occultation light curves similar to those of Triton and Pluto when we varied the (1) boundary radius, (2) data-averaging interval, (3) atmospheric shell thickness (Fig. 2), (4) thermal gradient in the occulting body's atmosphere, (5) level of light-curve noise, and (6) background level (by introducing systematic errors into the zero stellar flux level).

We note that our method presents three opportunities for averaging or binning the data and the intermediate results. First, the stellar fluxes can be averaged in the time domain 


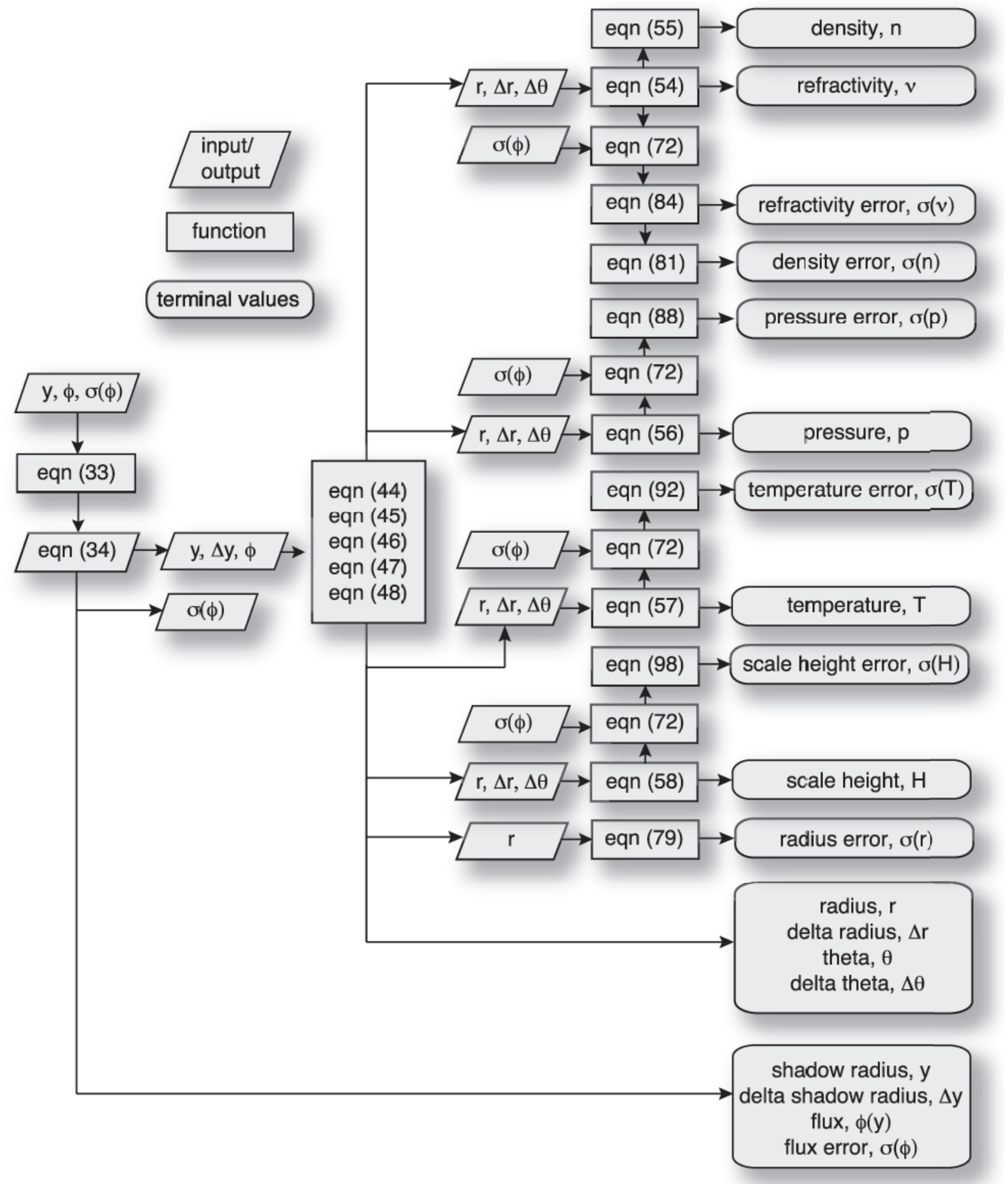

FIG. 3.-Flowchart for the inversion calculation. Calculation generally flows from left to right, as the initial data, represented by eq. (32), are processed to the complete inversion profiles on the right. Note the recurrence of certain key equations, such as eq. (72), which appears as part of the error calculations in each segment of the output.

prior to constructing the data set represented by equation (32). Secondly, the data set represented by equation (32) can be averaged further after being transformed from a function of time to a function of the $y$-coordinate in the observer plane. Finally, after using the fluxes to construct the atmospheric shells with equations (44) through (48), the adjacent shells can be binned into thicker shells if desired.

\subsection{Reference Point and Scaling of the Test Results}

A common reference point within the occulting body's atmosphere for occultation results is the radius of half-light (§3.3 and Paper I), and the widely used distance scale is the pressure scale height (Paper I). Regardless of the absolute radius interval within the occulting body's atmosphere 
covered by the inversion, or the actual radius range covered by individual data points, the parameter that controls the rate at which various errors grow is the number of scale heights relative to the reference point. Tests conducted by rescaling all parameters resulting from changing the scale height indeed give virtually identical results. Hence, we have added a "scale heights above half-light" scale to our plots (along with the radius scale) to facilitate scaling our test results to other cases of interest.

Also, as will be discussed in $\S 6.3$, the errors in the quantities determined by the inversion scale inversely with the signal-to-noise ratio (per scale height interval) of the occultation light curve. This can be seen in equation (72), where the variance of the stellar fluxes is a multiplier in the second summation term. The same scaling applies to the first (double) summation term in equation (72), when the data are accurate enough for the linear approximation for the least-squares fitting of a nonlinear light-curve model to be valid. This is almost always the case.

\subsection{Tests with Noiseless Synthetic Light Curves \\ 6.2.1. Standard Test Case}

A standard test case was established to confirm the basic accuracy of the method in reproducing an expected thermal profile. The Paper I small-body atmospheric model was used to generate the standard test-case data. The model atmosphere for the standard case is isothermal (i.e., $b=0$ ) with a temperature of $80 \mathrm{~K}$, a $30 \mathrm{AU}$ body-observer distance, a pressure scale height (at half-light) of $30 \mathrm{~km}$, and a half-light radius of $1200 \mathrm{~km}$. The full resolution of the data $(\Delta y=0.5 \mathrm{~km}$ per data point) was used, and the resulting $\Delta r$ 's were binned to a minimum shell size of $1.0 \mathrm{~km}$. A complete tabulation of the parameters used to generate the light-curve data from the Paper I model is given in Table 1.

A limitation of our standard test case is that the model used to generate the synthetic light curve for our tests is not exact but involves asymptotic series expansions that can be carried out to different orders (see Paper I). In order to understand the limitation that our light-curve model imposes on our inversion testing, we generated light curves with different orders of the asymptotic series and inverted them. Figure 4 shows these results. In the top panel, we see a large improvement in the accuracy of the light curve by using just the first term of the series. In the greatly expanded scale of the bottom panel, we see further improvement if the series order is increased to 2 , but not much improvement beyond that. Hence we elected to use a series expansion order of 2 for our standard test case. This case is plotted as a heavy line in the top and bottom panels of Figure 4, as well as in the remaining test-case figures.

The remaining differences from an isothermal temperature are due to a combination of (1) limitations in the numerical inversion process (most notably the approximation of integrals by sums), (2) errors in the synthetic data as generated from the Paper I model, and (3) possible (small) systematic errors due to the one-sided (rather than twosided) numerical derivatives. If desired, the second effect could be eliminated by inverting a sample light curve generated from an exact analytical solution to a known thermal profile (Eshleman \& Gurrola 1993; Chamberlain \& Elliot 1997), and the third effect could be eliminated with the use of two-sided derivatives (at some expense of computation time). However, the errors in our standard test case are well below $0.01 \%$ and not important for practical purposes: in the bottom panel of Figure 4 we see that the inversion accurately reproduces the $80 \mathrm{~K}$ isothermal profile of the model atmosphere within $0.01 \mathrm{~K}$. The average temperature given by all points of the inverted profile is $79.997 \mathrm{~K}$, very close to the model value, although the final points of the inversion converge to a slightly closer value of $79.998 \mathrm{~K}$.

\subsubsection{Isothermal Tests}

Variations of the inversion parameters from their values in this standard case were carried out with the same data as used in the standard case. Each parameter was changed independently, with the values for the other parameters kept at their values in the standard case. Table 2 gives the values used in the trial inversions for synthetic light curves that were calculated with the

TABLE 1

Parameters Used for the Standard Test Case

\begin{tabular}{|c|c|c|}
\hline Parameter & Value & Explanation \\
\hline \multicolumn{3}{|l|}{ Explicit model parameters: } \\
\hline 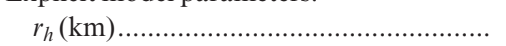 & 1200 & Half-light radius of occulting atmosphere \\
\hline 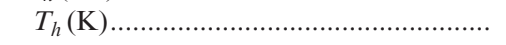 & 80 & Temperature of occulting atmosphere at half-light \\
\hline 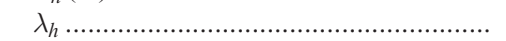 & 40 & Energy ratio at half-light (Paper I) \\
\hline$b$ & 0 & Exponent of temperature gradient in occulting atmosphere (isothermal for $b=0$ ) \\
\hline 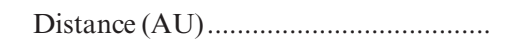 & 30 & Distance between occulting body and observer \\
\hline Atmospheric composition ....................... & $\mathrm{N}_{2}$ & \\
\hline Mean molecular weight $(\mathrm{amu})$............... & 28.01 & \\
\hline Refractivity at STP $\left(\times 10^{-4}\right) \ldots \ldots \ldots \ldots \ldots \ldots$ & 2.98 & \\
\hline \multicolumn{3}{|l|}{ Derived model parameters: } \\
\hline 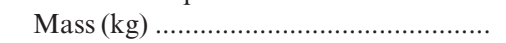 & $1.70833 \times 10^{22}$ & Mass of occulting body \\
\hline Scale height at half-light $(\mathrm{km}) \ldots \ldots \ldots \ldots \ldots . .$. & 30 & \\
\hline \multicolumn{3}{|l|}{ Inversion parameters: } \\
\hline 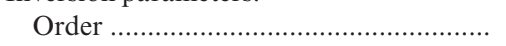 & 2 & Order of asymptotic series expansions in boundary model calculations (Paper I) \\
\hline Radial resolution, $\Delta y(\mathrm{~km}) \ldots \ldots \ldots \ldots \ldots \ldots \ldots$ & 0.5 & Resolution of synthetic data in observer plane \\
\hline Shell thickness, $\Delta r(\mathrm{~km}) \ldots \ldots \ldots \ldots \ldots \ldots \ldots \ldots$ & 1.0 & Minimum thickness of inversion shells in occulting body's atmosphere \\
\hline Flux level at boundary index ................. & 0.5 & Stellar flux level at which inversion begins \\
\hline Points in the boundary region................ & 860 & \\
\hline Points in the inversion region.................. & 80 & \\
\hline
\end{tabular}



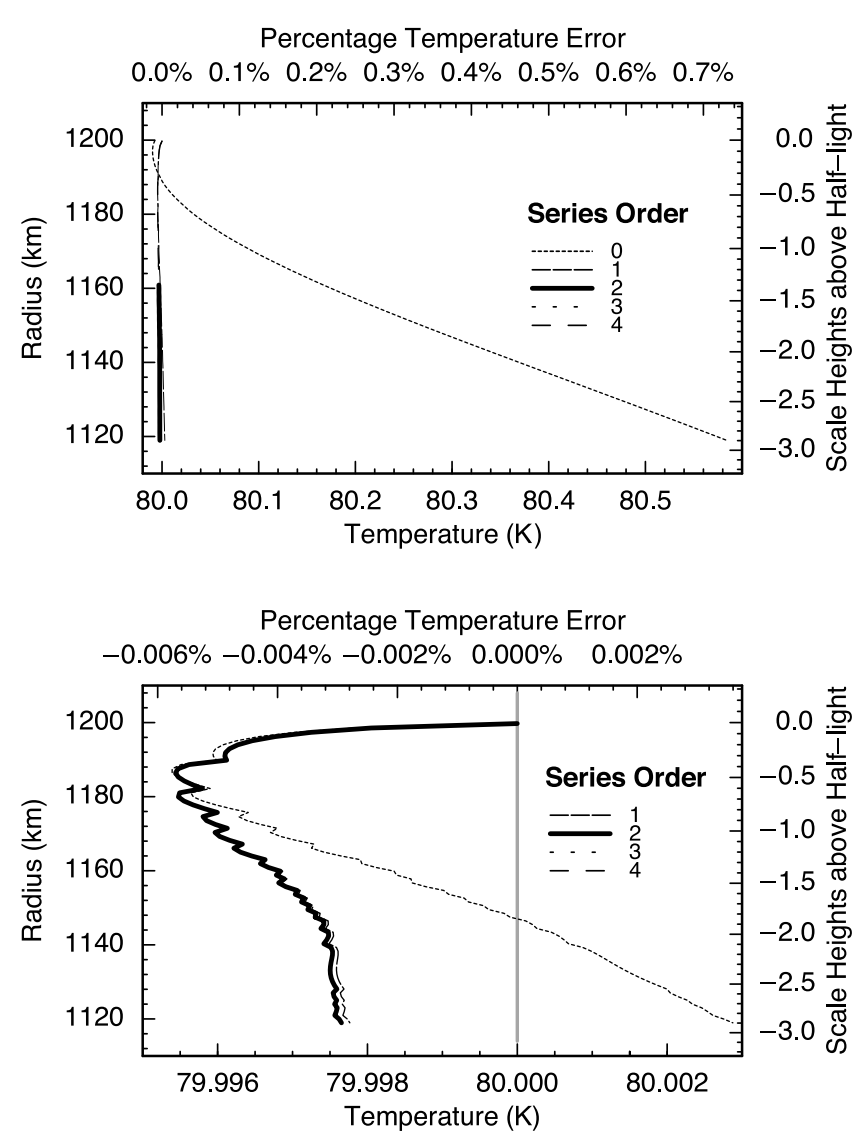

FIG. 4.-Isothermal test cases: different orders for series expansions. This figure displays the results of inversions of the standard test case for different orders of the asymptotic series expansion (eqs. [64] and [67] in $\S 3.3$ ) for the light-curve model (Paper I) used for these tests. Both panels show a plot of temperature (abscissa) vs. radius (ordinate). The right ordinate scale shows the scale heights above half-light equivalent to the radius scale on the left, and the top scale represents the percentage deviation of the calculated temperature from the model temperature of $80 \mathrm{~K}$ (shaded vertical line). Different line styles have been plotted instead of discrete temperature points to make the results for different inversions more distinguishable. The heavy solid line represents the standard isothermal test case in both panels. The bottom panel gives an expanded view of the left portion of the top panel, which illustrates the difference of the temperature profile for the first-order expansion from that for the second-order expansion (standard test case). The curves for the third- and fourth-order results are nearly indistinguishable from the second-order result. Note the extremely expanded temperature scale of the bottom panel. small-body model described in Paper I, where the parameter values for the standard test case are displayed in boldface. All columns but the last refer to parameter variations used with noiseless light curves. Each value in the table indicates one inversion trial in which the tabulated parameter was fixed as given, with all other parameters set to their standard values.

The three panels in Figure 5 show the temperature profiles used to characterize the sensitivity of the inversion results to (1) different stellar flux levels used to specify the inversion boundary (which effectively specify the inversion boundary radius, $r_{b}$ ) (2) different averaging intervals of the data in the observer plane ( $y$-coordinate), and (3) different minimum shell sizes within the occulting body's atmosphere ( $r$-coordinate). As seen in the top panel, changing the value of the boundary flux produces only a small error in the resulting temperature-much less than $0.01 \%$ for all cases considered. In the next set of test cases (Fig. 5, middle), we averaged the data over different intervals in the observer plane, which we have expressed in terms of "points per scale height" for the averaged data. The extreme case for this test was to average the data over $30 \mathrm{~km}$ intervals. Although this distance equals 1 scale height of the occulting body's atmosphere, the actual interval sampled within the occulting body's atmosphere is only $15 \mathrm{~km}$ at half-light-because of differential refraction - and the corresponding distance within the occulting body's atmosphere becomes progressively less as the stellar flux decreases (see eq. [2]). Even for averaging the data to one point per scale height (in the observer plane), the maximum error in the temperature profile resulting from the inversion is less than $0.5 \%$.

A different way to average the data is to bin the atmospheric shells, and the effects of this procedure are illustrated in the bottom panel of Figure 5. As with averaging the data in the observer plane, the temperature errors are small and reach a maximum error of less than $0.7 \%$ for a binning of two points per scale height. This extreme binning corresponds to $15 \mathrm{~km}$ (half a scale height) in the body's atmosphere.

Table 3 gives the representative values of average temperature inverted and final convergence temperature for selected inversion test cases just described. For all cases, the actual temperature is the standard value of $80 \mathrm{~K}$. The

TABLE 2

Parameters for Test Cases

\begin{tabular}{|c|c|c|c|c|c|c|}
\hline $\begin{array}{l}\text { Inversion Trial } \\
\text { Series }\end{array}$ & $\begin{array}{l}\text { Series Expansion } \\
\text { Order }\end{array}$ & $\begin{array}{l}\text { Data Resolution, }{ }^{\mathrm{a}} y \\
(\mathrm{~km})\end{array}$ & $\begin{array}{l}\text { Shell Size, }{ }^{\mathrm{a}} \Delta r \\
\quad(\mathrm{~km})\end{array}$ & $\begin{array}{c}\text { Flux at Boundary } \\
\text { Radius }\end{array}$ & $\begin{array}{c}\text { Thermal Gradient } \\
\text { Parameter, } b\end{array}$ & $\begin{array}{l}\text { Signal-to-Noise } \\
\text { Ratio, }(\mathrm{S} / \mathrm{N})_{H}{ }^{\mathrm{b}}\end{array}$ \\
\hline $1 \ldots \ldots \ldots \ldots \ldots \ldots \ldots \ldots$ & 0 & 0.5 & 1 & 0.3 & -6 & $\infty$ \\
\hline 2 & 1 & 1 & 2 & 0.4 & -3 & 500 \\
\hline З & 2 & 2 & 6 & 0.5 & $\mathbf{0}$ & 200 \\
\hline $4 \ldots \ldots \ldots \ldots \ldots \ldots \ldots \ldots$ & 3 & 3 & 12 & 0.6 & 3 & 100 \\
\hline 5 & 4 & 5 & 20 & 0.7 & 6 & 50 \\
\hline 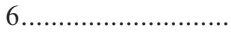 & $\ldots$ & 10 & 30 & 0.8 & 9 & 20 \\
\hline 7 & $\ldots$ & 30 & $\ldots$ & $\ldots$ & $\ldots$ & $\ldots$ \\
\hline 8. & $\ldots$ & 60 & $\ldots$ & $\ldots$ & $\ldots$ & $\ldots$ \\
\hline
\end{tabular}

NotE.-Each tabulated value represents a single trial, and parameters for the standard test case are in boldface. For that trial, the tabulated value was fixed as listed and all other values were fixed to their value for the standard case given in boldface. The series expansion order refers to eqs. (64) and (67) describing the model, and the thermal gradient parameter $b$ refers to eq. (59) (see Paper I).

a Scale height is $30 \mathrm{~km}$.

b Background-limited. 

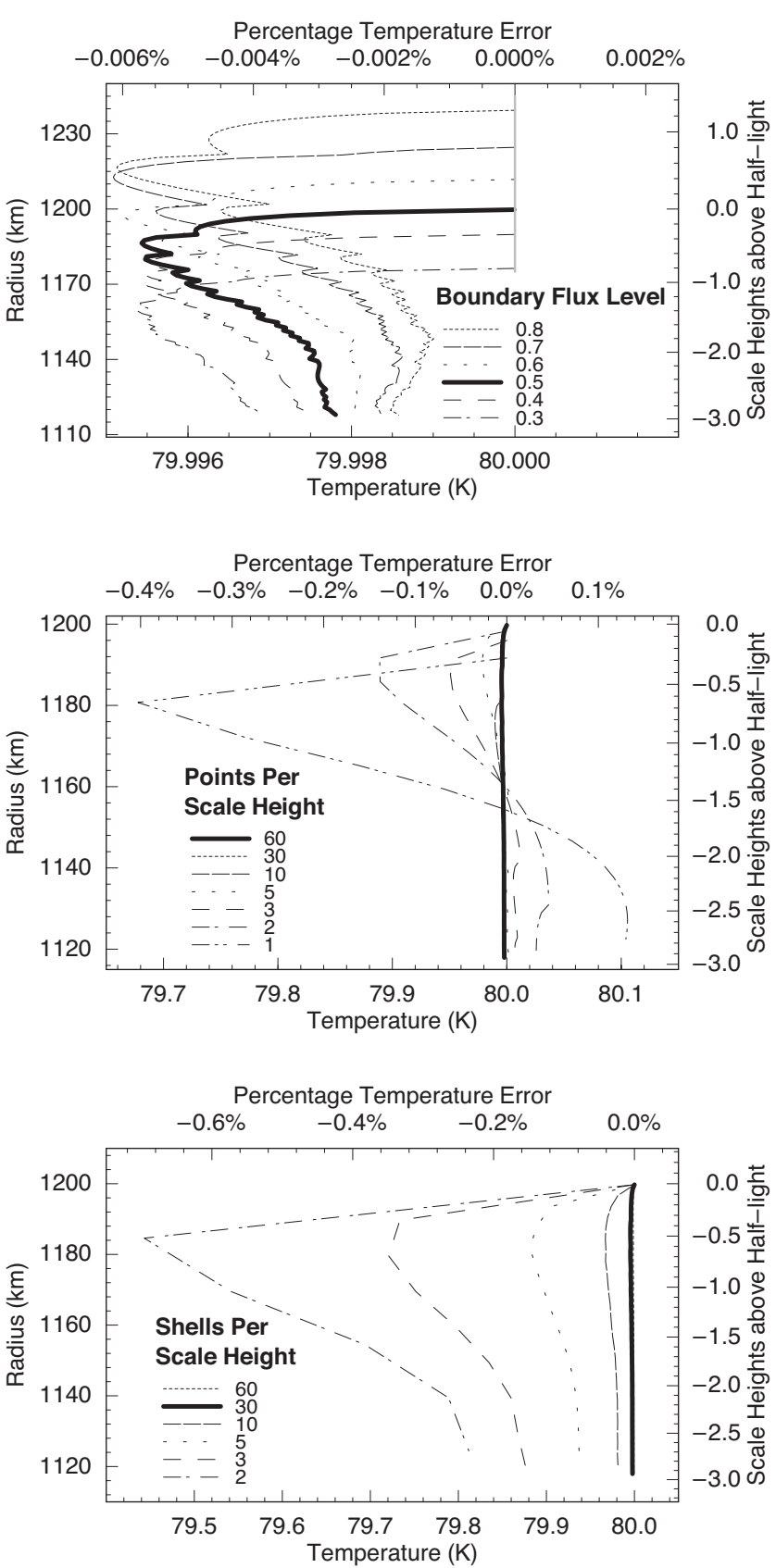

FIG. 5.-Isothermal test cases: (1) different boundary flux levels, (2) points per scale height in the observer plane, and (3) shells per scale height in the body plane. The right scale shows the scale heights above half-light equivalent of the radius scale, and the top scale represents the deviation of the calculated temperature from the model temperature of $80 \mathrm{~K}$ (shaded vertical line). The three panels give the results of the noiseless test cases given in the second through fourth columns of Table 2. In each panel, the temperature (abscissa) is plotted vs. radius (ordinate). Top: Temperature profiles for different selections of boundary flux level, which is defined as the light-curve flux for the boundary radius $r_{b}$. Middle: Inversion profiles for different amounts of averaging of the data in the $y$-domain (observer plane), expressed as the number of points per scale height. Bottom: Temperature profiles for different amounts of shell binning in the body-plane domain, expressed as shells per scale height. The inversion has been plotted with dashed lines instead of discrete data points to make the curves more distinguishable. In each case the heavy solid line represents the standard isothermal test case (see text for discussion).

inversion procedure yields remarkably accurate temperatures, even when the data are averaged over large intervals in the observer plane or the atmospheric shells have been binned to a thickness of half a scale height.

\subsubsection{Nonisothermal Tests}

In order to ensure that our results are applicable to a wider set of data than the isothermal test cases establish above, we inverted a test series of data that had different thermal gradients in the temperature profiles. The data for these test cases were generated using the Paper I model as before, with the addition of a nonzero $b$-parameter. Also, the equivalent isothermal lambda parameter, $\lambda_{\mathrm{hi}}$, was adjusted according to equation (62) for each case to keep the actual binding ratio $\lambda_{h}$ constant across all test cases, resulting in a half-light temperature of $80 \mathrm{~K}$ for all synthetic data sets (as we did for the isothermal test cases).

Figure 6 shows the results of inversion of six test cases with thermal gradient parameters ranging from $b=-6$ to $b=9$. This range encompasses the space of thermal gradients found for the atmospheres of Pluto and Triton. In each case, the initial point in the inversion (from the boundary condition) starts at the expected temperature of $80 \mathrm{~K}$, and then as the temperature changes, the expected value calculated from equation (59) diverges from the inverted result initially and then its agreement improves. In the worst case tested $(b=9)$, the maximum divergence is still within $0.5 \%$, a value well within our expected tolerances from the test cases with noise added.

When the thermal gradient test-case data were generated, the choice of available scope for variations of $b$ was limited strongly by the expansion order of the asymptotic series in the Paper I model. For the displayed cases, an expansion order of 2 was used, to provide consistency with the standard isothermal test case. Higher values of $b$ than those displayed did not converge for this choice of expansion order, so these could be tested only with lower order expansions (but yielded similar results). The clear trend in maximum divergence from the expected temperature increasing with $b$ persisted through all trials and thus indicates that this divergence is a demonstration of deficiencies in the series expansion methods chosen for the Paper I model rather than an inherent problem with the inversion procedure. Remedies

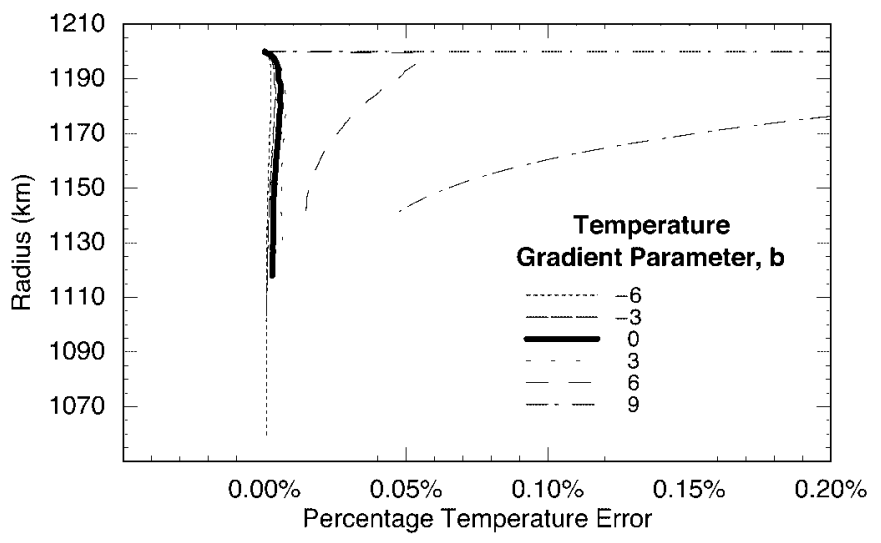

FIG. 6.-Nonisothermal test cases: residuals in temperature profile inversion with varied levels of the temperature gradient parameter $b$. The percentage errors in the temperature profiles (abscissa) with different temperature power indexes are plotted vs. the radius scale (ordinate). The figure has been plotted with dashed lines instead of discrete data points to make the curves more distinguishable. The heavy solid line represents the percentage temperature errors for the standard isothermal test case without noise. Note that as the temperature power index decreases, the temperature profiles reach deeper into the atmosphere. For the cases shown, the largest deviations from the $80 \mathrm{~K}$ isothermal temperature occur for the $b=9$ case, which reaches a maximum error of $+0.48 \%$ (off scale to the right). 
TABLE 3

Comparison of Results From NoISEless InVErsion Test Cases

\begin{tabular}{ccccc}
\hline \hline $\begin{array}{c}\text { Inversion } \\
\text { Test Case }\end{array}$ & $\begin{array}{c}\text { Series No. } \\
\text { (Table 2) }\end{array}$ & $\begin{array}{c}\text { Average Temperature } \\
(\mathrm{K})\end{array}$ & $\begin{array}{c}\text { Convergence Temperature } \\
(\mathrm{K})\end{array}$ & $\begin{array}{c}\text { Maximum Residual } \\
(\mathrm{K})\end{array}$ \\
\hline Standard inversion ................ & $\ldots$ & 79.997 & 79.998 & 0.004 \\
Boundary radius flux............... & 1 & 79.997 & 79.998 & 0.005 \\
& 4 & 79.997 & 79.998 & 0.005 \\
& 6 & 79.998 & 79.999 & 0.005 \\
Data resolution ................... & 2 & 79.99 & 80.01 & 0.03 \\
& 4 & 79.96 & 80.03 & 0.05 \\
Shell size ............................. & 7 & 79.88 & 80.10 & 0.32 \\
& 2 & 79.99 & 79.98 & 0.02 \\
& 4 & 79.96 & 79.88 & 0.26 \\
\hline
\end{tabular}

for this shortcoming of our boundary condition will be discussed in $\S 7.7$.

\subsection{Tests with Noisy Synthetic Light Curves}

Once the basic accuracy of the inversion method was shown on the suite of test cases with noiseless synthetic data, further tests were conducted with white Gaussian noise added to the noiseless light curves. The added noise had a constant rms level, independent of the stellar flux, which describes a "background limited" situation (French et al. 1978); our inversion method, of course, accommodates cases that are not background limited, since the standard deviation of each light-curve point can be specified independently (see eq. [32]). The standard deviation of the noise - averaged over a distance in the observer plane equal to a scale height in the occulting body's atmosphere - was 0.01 of the normalized stellar flux. This quantity was introduced by French et al. (1978) as an rms error per scale height, and it provides a convenient way to compare the quality of atmospheric occultation light curves. The reciprocal of this quantity is the signal-to-noise ratio $(\mathrm{S} / \mathrm{N})_{H}$ of the synthetic occultation light curve (100 "per scale height" for this case). The $(\mathrm{S} / \mathrm{N})_{H}$ can most easily be calculated from the pre- and post-occultation sections of the light curve by dividing the $(\mathrm{S} / \mathrm{N})$ of a single point by the square root of the number of points in a scale height (in the observer plane). If $\sigma$ is the standard deviation of the normalized stellar flux averaged over an interval $\Delta y$ in the observer plane outside the occultation (this can be calculated by multiplying the time resolution of the light curve by the shadow velocity perpendicular to the limb of the occulting body) and $H$ is the scale height of the occulting body's atmosphere at half-light, then $(\mathrm{S} / \mathrm{N})_{H}$ is given by

$$
(\mathrm{S} / \mathrm{N})_{H}=\sigma^{-1} \sqrt{H / \Delta y} .
$$

The noisy light curve was inverted with the error propagation calculations discussed in $\S 4$, which resulted in the temperature profile displayed in Figure 7 . Note that the formal error bars in the temperatures show good agreement with the deviations of the profile from the isothermal temperature of $80 \mathrm{~K}$. The reliability of these formal errors, as calculated directly from the inversion using the procedures given in $\S 4$, was further tested by repeating the inversion of the standard case with 25 different, randomly generated noise samples. For each atmospheric level, the error calcu- lated from the scatter of the 25 different sample inversions matched the errors generated by the analytic treatment to within $18 \%$ for the worst case. These temperature profiles were ranked according to their deviation from the $80 \mathrm{~K}$ input temperature, and the median was selected for display. The selected case is displayed in Figure 8, where one can see the close agreement between the calculated errors of a single noisy sample and the scatter of inverted pressures of 25 noisy samples in that set.

The effect of varying signal-to-noise levels were tested with $(\mathrm{S} / \mathrm{N})_{H}$ values ranging from 20 through 1000 , a range that includes the Pluto and Triton light curves used as examples in $\S \S 8$ and 9. These results are displayed in Figure 9, where the top panel shows a single inversion profile for a light curve of the six $(\mathrm{S} / \mathrm{N})_{H}$ values tested and the bottom panel shows the upper and lower error envelopes for 1 standard deviation of the temperature. Values of $(\mathrm{S} / \mathrm{N})_{H}$ higher than 200 reproduce inversion profiles commensurate with that of the standard noiseless test case to within $2 \%$. As

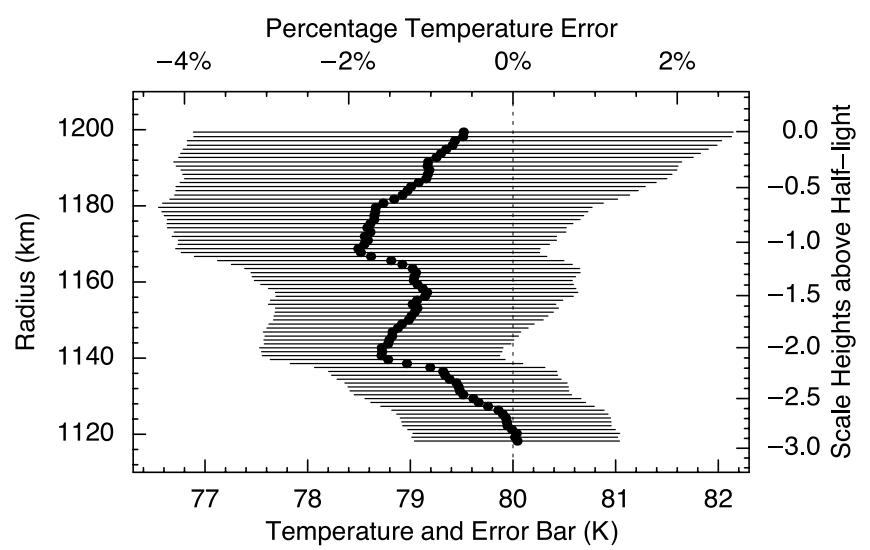

FIG. 7.- Standard test case with noise. Temperature (abscissa) is plotted vs. radius (ordinate) for a representative isothermal test case with the addition of Gaussian background noise that has a standard deviation of $1 \%$ per scale height $\left[(\mathrm{S} / \mathrm{N})_{H}=100\right]$. This case was chosen as the median-quality example from a set of 25 independent trials (see text). The right scale shows the scale heights above half-light equivalent of the radius scale, and the top scale represents the deviation of the calculated temperature from the expected temperature of $80 \mathrm{~K}$. Note the expanded scale, given to show the structure of the residuals from the model temperature of $80 \mathrm{~K}$ (dashed vertical line). The apparent temperature gradient and thermal undulations are all artifacts created by the noise added to the isothermal model. The apparent undulations in the temperature profile are due to the correlation of the temperature errors. (see text). 


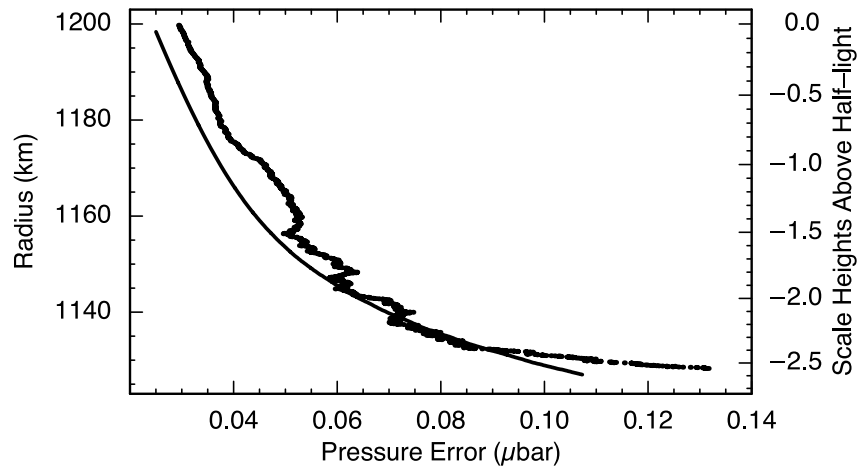

FIG. 8. - Calculated pressure errors vs. actual scatter. The jagged curve (which is a mostly unresolved plot of individual points) represents the standard deviation of 25 inversion pressure profiles calculated from the standard isothermal light curve, each with a different random noise sample added. The smooth curve is the error in the pressure profile that was calculated from a selected sample (out of the 25) with the equations presented in this paper. This curve is plotted as a line instead of discrete data points so that the two curves may be more easily distinguished. Note that the calculated pressure error closely matches the actual scatter of the pressure values. Carrying out this calculation with additional noise samples should improve the agreement.

$(\mathrm{S} / \mathrm{N})_{H}$ drops, the inversion profiles become progressively less accurate, inversely proportional to $(\mathrm{S} / \mathrm{N})_{H}$. The temperature profile for an $(\mathrm{S} / \mathrm{N})_{H}$ of only 20 has a maximum rms error envelope that does not exceed $15 \%$. For each value of $(\mathrm{S} / \mathrm{N})_{H}$, the median case was selected for plotting in Figure 9.

We tested the effect that changes in the inversion boundary radius have on the boundary and summation errors, and the results of this test are displayed in Figure 10. Here we have specified the boundary radius with the corresponding normalized stellar flux level, ranging from 0.3 to 0.8 . At the boundary, the error contribution from the summation terms is strictly zero, and the summation family of curves all begin at zero on the left side of the figure. The family of curves that give the boundary contribution to the error traverse the figure from upper right to lower left, where they cross the summation contribution curves at 2.5-3.0 scale heights below half-light. Though the inversions start at different flux levels in the light curve for each case, the summation errors in the final inversion points all converge to similar values, which demonstrates that the inversion boundary radius has little effect on the final summation errors.

The same differences in boundary flux level affect the boundary errors more strongly. Inversions starting higher in the light curve generate larger boundary errors, and the difference in the errors persists throughout the inversion region. Thus, in our standard case where the inversion boundary index corresponds to a normalized stellar flux level of 0.5 , the boundary error at the bottom of the inversion region is roughly half the summation error. However, in the case where the boundary flux level is as high as 0.8 , the boundary error at 3 scale heights below half-light is larger than half the summation error.

\subsection{Computation Time}

We ran our standard case with noise (see $\S$ 6.3) using data sets of different lengths in order to gain an understanding of the time required for the inversion calculations. These runs were performed on a Macintosh G3 PowerBook with a 500 MHz CPU. For test runs with 39, 81, 166, and 333 points,
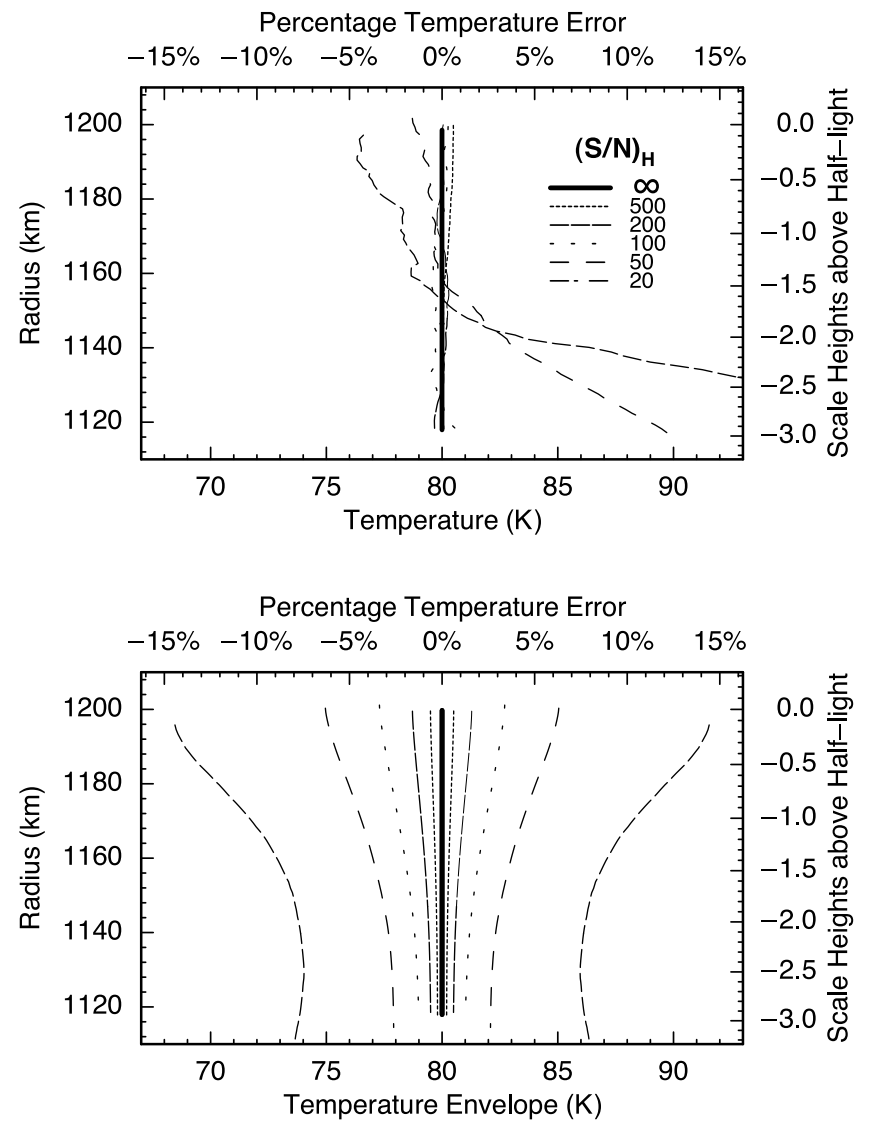

FIG. 9.--Isothermal test cases with noise: differences in signal-to-noise ratio. Shown are the inverted temperature profiles of a representative test case generated as in Fig. 7, but for different levels of random Gaussian background noise added. The no-noise curve $\left[(\mathrm{S} / \mathrm{N})_{H}=\infty\right]$ is the standard test case (the solid lines in the three panels of Fig. 5), plotted here for comparison. In each panel, temperature (abscissa) is plotted vs. radius (ordinate). The top panel shows a temperature profile for a sample inversion for each value of $(\mathrm{S} / \mathrm{N})_{H}$, while the bottom panel shows their 1 standard deviation error envelopes. In both panels, the right scale shows the scale heights above half-light equivalent of the radius scale, and the top scale represents the deviation of the calculated temperature from the model temperature of $80 \mathrm{~K}$. The figure was plotted with dashed lines instead of discrete data points to make the curves more distinguishable. Note that the error envelopes decrease in inverse proportion to $(\mathrm{S} / \mathrm{N})_{H}$. Each profile would be expected to lie outside its 1 standard deviation error envelope for approximately one-third of its radial extent, on average. The gradients and other apparent thermal structure in the sample profiles are the artifacts of the light-curve noise.

the execution times for the major parts of the inversion calculations are listed in Table 4. These numbers of binned points increase by approximately a factor of 2 for each successive selection. From Table 4, we can see that the time it takes to run the model fit and establish the initial conditions and associated errors is very short compared with the inversion and error calculations. The error calculations take by far the longest time to run. We can see also that the inversion time increases approximately linearly with the number of points in the inversion region, while the computation time for the errors increases approximately quadratically with the number of points.

\section{DISCUSSION OF THE INVERSION METHOD}

In this section, we discuss several issues concerning the inversion method before applying it to occultation light 
TABLE 4

TIMES FOR THE INVERSION COMPUTATIONS

\begin{tabular}{|c|c|c|c|c|c|}
\hline $\begin{array}{c}\text { Model Fit } \\
\text { (Boundary) Points }\end{array}$ & $\begin{array}{l}\text { Inversion } \\
\text { Points }\end{array}$ & $\begin{array}{c}\text { Model Fit } \\
\text { (Boundary) } \\
\text { (s) }\end{array}$ & Inversion $^{\mathrm{a}}$ & Errors $^{\mathrm{b}}$ & Total $^{\mathrm{b}}$ \\
\hline 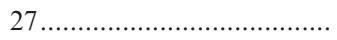 & 40 & 4 & $0: 18$ & $00: 18: 40$ & 00:19:02 \\
\hline ……...... & 82 & 7 & $0: 53$ & 01:21:02 & 01:22:02 \\
\hline 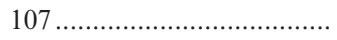 & 167 & 9 & $1: 28$ & $06: 13: 31$ & 06:15:08 \\
\hline 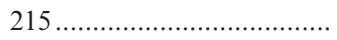 & 334 & 17 & $3: 05$ & $28: 55: 03$ & $28: 58: 25$ \\
\hline
\end{tabular}

a Minutes and seconds.

${ }^{\mathrm{b}}$ Hours, minutes, and seconds.

curves for Pluto and Triton in $\S \S 8$ and 9. The progression of topics proceeds from those pertaining to the immediate application of the inversion method as described, then to its limitations, and finally to ideas for future development.

\subsection{Scope of the Test Cases}

The test cases described here provide a basic probe of the large parameter space associated with the inversion procedure, but they are by no means exhaustive. As with any complex data analysis, one must exercise "constant vigilance" for potential problems with the method, its implementation, and the data set itself. In this regard, we recommend that when inverting an occultation light curve, one generate a set of synthetic data that bracket the parameter space relevant to the data set being analyzed. To these data sets one should add different noise samples that have similar statistical characteristics to the noise in the data. The results of these tests can give one a sense of the reliability of inversion results for an occultation light curve of interest.

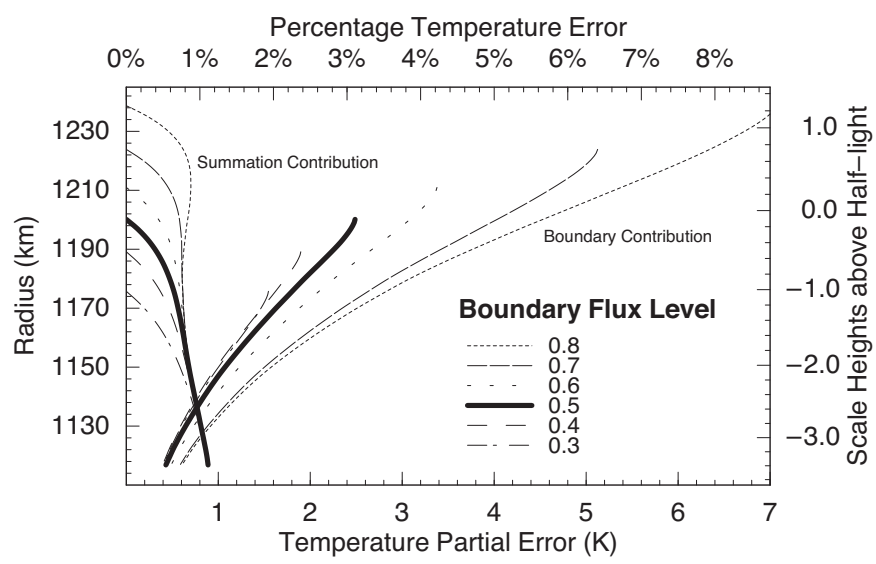

FIG. 10.-Isothermal test cases with noise: temperature profile errors for different boundary flux levels. This figure displays the boundary and summation errors for different inversion boundary radii, as parameterized by the boundary flux level. The figure has been plotted with dashed lines instead of discrete data points to make the curves more distinguishable. The right scale shows scale heights above half-light equivalent of the radius scale on the left side. The heavy solid lines represent the errors for the standard isothermal test case with noise. The group of curves starting at the left axis represent the summation errors, while the other group of curves show the boundary errors. Note that all summations errors converge to a common value near the bottom of the curves - indicating that the boundary flux level for the inversion has little to no effect on summation errors in the temperatures for the smallest radii. This behavior is in contrast with the boundary errors, which are greatly affected by the boundary flux level, and, consequently, by the errors in the boundary model fit. The greater errors from the boundary integral for higher boundary fluxes persist to the lowest radii.

\subsection{What Light Curves Should Be Inverted?}

In the analysis of stellar occultation light curves, the question arises as to when one would consider an occultation light curve "too noisy" for inversion and limit the analysis to model fitting. We can use the test results for our inversion method to gain insight into this issue. Our discussion in this section will be limited to the effects of only random noise, but we must bear in mind that a systematic error in the zero stellar flux level (as discussed in $\S$ 7.4.1) can, in some cases, be the limiting factor in the accuracy of inversion results, rather than random noise in the light curve.

For the lowest signal-to-noise ratio $\left[(\mathrm{S} / \mathrm{N})_{H}=20\right]$ considered for the cases plotted in Figure 9, the error envelope in the bottom panel indicates a minimum percentage error in the temperature of about $7 \%$. On the other hand, if we fit the light curve with the small-body model from Paper I that was used to generate the noiseless light curve, the formal error in the temperature is $\pm 5 \%$ (Table 5). Hence the percentage errors in the temperature from inversion and from model fitting are comparable, although the model fitting yields a more precise result. However, in principle, more information is available from the inversion results, since the inversion yields an entire temperature profile rather than a single temperature (and possibly a temperature gradient, as would be available from the Paper I model).

As an example, we ask what information we can glean from the sample temperature profile presented in the top panel of Figure 9 for the $(\mathrm{S} / \mathrm{N})_{H}=20$ case. The temperature profile lies within the error envelope for approximately two-thirds of its radial extent - which is roughly what would be expected for an error envelope of 1 standard deviation. However, one must avoid the temptation to overinterpret this temperature profile, since errors in adjacent temperatures from the inversion are highly correlated. This gives the

TABLE 5

Comparison of InVersion with Isothermal Model Fitting

\begin{tabular}{|c|c|c|c|}
\hline$(\mathrm{S} / \mathrm{N})_{H}$ & $\begin{array}{c}\text { Inversion } \\
\text { Temperature }^{\mathrm{a}} \\
(\mathrm{K})\end{array}$ & $\begin{array}{c}\text { Minimum } \\
\text { Inversion Error } \\
\text { (K) }\end{array}$ & $\begin{array}{l}\text { Model Fit } \\
\text { Temperature } \\
\text { (K) }\end{array}$ \\
\hline$\ldots \ldots$ & 88.1 & 6.0 & $76.1 \pm 4.2$ \\
\hline ........... & 81.9 & 2.1 & $82.3 \pm 1.7$ \\
\hline $100 \ldots \ldots \ldots \ldots \ldots \ldots$ & 79.9 & 1.0 & $80.5 \pm 0.8$ \\
\hline $200 \ldots \ldots \ldots \ldots \ldots$ & 80.0 & 0.5 & $79.5 \pm 0.4$ \\
\hline $500 \ldots \ldots \ldots \ldots \ldots \ldots$ & 80.2 & 0.2 & $79.7 \pm 0.1$ \\
\hline
\end{tabular}

NotE.-For a noisy synthetic light curve generated for an isothermal atmosphere with a temperature of $80 \mathrm{~K}$.

${ }^{a}$ Unweighted mean of the temperatures of all inversion points. 
impression of thermal gradients and wavelike structures, when in fact the occulting atmosphere is isothermal. A further example of this behavior is the temperature profile displayed in Figure 7, which also has been derived from an isothermal atmospheric model. We have not investigated the correlations in the temperature errors in detail, but crudely one might expect that we get an approximately independent point per scale height. Hence if the temperature profile extends over several scale heights, then one should expect that portions of it will lie outside the 1 standard deviation error envelope. This dictates that the criterion for "real structure" in a temperature profile should be set conservatively.

Rather than just being conservative and having lingering doubts about the validity of thermal structure derived from inversion, we can suggest a direct approach for separating real thermal structure from the effects of correlated errors. Our prescription for deciding what is real and what is due to correlated errors is to simply fit a model to the light curve and then examine the residuals from the model fit. If one can establish that the residuals have structure beyond white noise that is undoubtedly produced by the occulting body's atmosphere (and not due to effects of extinction in the occulting body's atmosphere or correlated noise in the light curve, such as the effects discussed in $\S 7.4 .3$ ), then the light curve warrants inversion. This approach puts the decision making of which features are real into the time domain, where the noise is uncorrelated (except for terrestrial scintillation, which can be significant for small telescope apertures), making the judgment somewhat easier.

So the answer to our original question of when inversion is warranted depends on a combination of the strength of the nonisothermal structure in the occulting body's atmosphere and the noise level in the data. If the atmosphere is truly isothermal, then all the information to be gleaned from the light curve is contained in the model fit, no matter how high the signal-to-noise ratio. On the other hand, if the atmosphere has strong gradients or other thermal structure, the inversion of even a relatively noisy light curve can yield useful information about the occulting body's atmosphere.

\subsection{Selection of Inversion Parameters}

The results of the tests of the inversion method described in $\S 6$ can be used as a guide for setting the calculation parameters used to carry out the inversion of an occultation light curve. The basic decisions to be made are (1) where to set the light-curve flux that corresponds to the boundary radius, (2) how much to average the data in the time domain, and (3) how much to bin the shells corresponding to different radii within the planetary atmosphere.

As can be seen in the top panel of Figure 5, in the absence of noise in the light curve, the numerical accuracy of the inversion is better than $0.01 \%$ no matter what flux level is chosen for the boundary. Hence, to glean the most information from the inversion one would set the boundary flux to a value near 1.0. However, when one considers a light curve with noise (see Fig. 10), the larger the flux level of the boundary, the greater are the errors in the temperature profiles. Furthermore, these larger errors in the temperature profiles persist to lower radii. It appears that large gains in the error level are made by setting the boundary flux at 0.5 rather than 0.8 (Fig. 10), whereas little is gained by setting the boundary flux below 0.5 . In cases where the occultation light curve shows significant deviations from the boundary model above the 0.5 flux level, one must set the boundary above these, in order to recover the corresponding atmospheric structure, and accept the larger errors in the inversion results.

Unless computation time (Table 4) is an issue, we recommend that the data be averaged in the time domain by only the amount necessary to generate positive stellar fluxes. This approach gives the maximum radial resolution of the resulting temperature, pressure, and number density profiles. We note that the radial resolution improves as the flux decreases, as a result of the shrinkage of the Fresnel zone for the stellar flux passing through the body's atmosphere, but ray crossing and the stellar diameter may limit the radial resolution ( $\S 7.5)$. If computation time is an issue, however, one may choose to reduce the number of data points involved in the inversion - which can be accomplished by averaging the data or by binning the radius shells. The results of our tests (Fig. 5, middle and bottom) indicate that these are roughly equivalent in terms of the numerical errors that are introduced into the results. Binning the radius shells provides a uniform spacing of the profile points in radius space, which is generally the preferred output. We note that numerical errors of $0.7 \%$ are introduced into the temperature profiles if radius shells are binned to only two points per scale height.

\subsection{Light-Curve Errors in Addition to Gaussian Noise}

Until now, we have considered only errors arising from (Gaussian) white noise in the fluxes, characterized by their standard deviations $\sigma\left(\phi_{i}\right)$. However, occultation light curves contain other sources of error that must be considered as well. The ones that we shall discuss here are systematic background error, variable background, correlated noise (e.g., atmospheric scintillation), and contamination from the far-limb flux.

\subsubsection{Systematic Background Error}

Perhaps the most common source of error beyond random noise is an incorrect normalization of the light curve. One can readily establish the full-scale level for the sum of the stellar flux and background flux (usually light from the occulting body, though there can be other sources as well), but establishing the correct background level usually proves to be more difficult. A systematic error in the background level introduces errors into the radius scale and the atmospheric parameters determined from inversion of the occultation light curve.

Figure 11 shows the results of inversion test cases with deliberate systematic background errors introduced, but with no random noise. Systematic errors in temperature profile have been plotted for various levels of erroneous background calibration. The systematic background error has been simulated by producing a data set (based on the Paper I model) with a baseline offset from its true value. We can see that the inversion method is very sensitive to this type of error. In the cases where the background level deviates by 0.05 in normalized stellar flux away from zero in either direction, the temperature at 1 scale height below half-light has a similar, but somewhat larger, fractional error. This fractional error grows approximately linearly with scale height below half-light, reaching about $25 \%$ at the end of the inversion. Even in the cases where the back- 


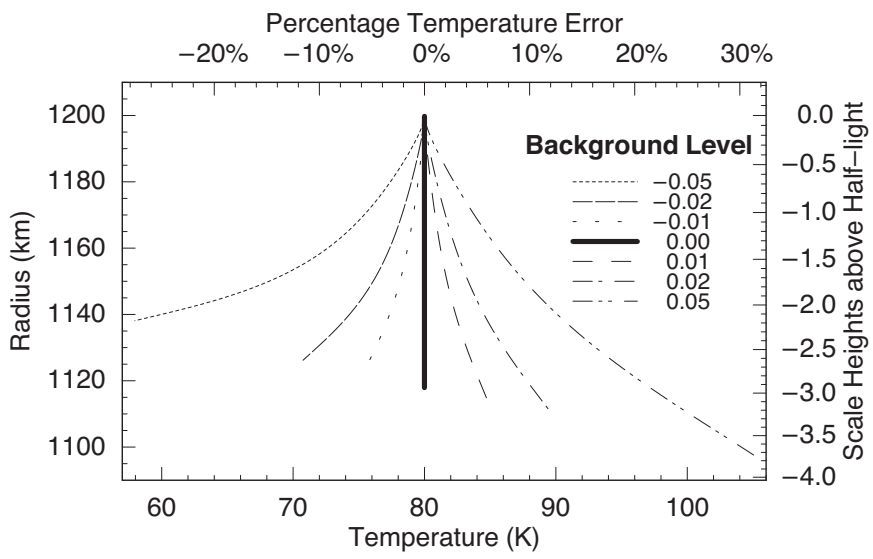

FIG. 11.-Isothermal test cases: systematic errors in the background level. The temperature profiles are plotted for the standard test case (the solid lines in the three panels of Fig. 5), as well as test cases with background levels systematically offset from the model zero level for the stellar flux, simulating incorrect calibration of the background. The right scale shows scale heights above half-light equivalent of the radius scale on the left side. The bottom scale indicates the actual calculated temperatures from the inversion, while the top scale represents the deviation in temperature from the model temperature of $80 \mathrm{~K}$. The figure was plotted with dashed lines instead of discrete data points to make the curves more distinguishable. The heavy solid line represents the standard isothermal test case with no offset in the zero stellar flux level. Note that errors in the background calibration introduce large systematic errors in the temperature profiles that grow with depth into the atmosphere.

ground level is in error by only 0.01 in normalized stellar flux, the final temperatures have a fractional error 3 scale heights below half-light of about 7\%, which is much larger than the errors generated by the other effects discussed here. The systematic trend in the lower ends of the curves in Figure 11 to lower radii reflects a corresponding error in radius scale that has been introduced by the systematic background error.

\subsubsection{Variable Background}

More problematic is a time-variable error in the background level that can arise from unmonitored variable amounts of background light that contaminate the occultation light curve. By "variable background" we mean unknown changes in the background level from integration interval to integration interval that could be introduced in the photometric reduction that was used to produce the normalized occultation light curve. In the case of Pluto, this effect can occur if Charon is near the edge of the aperture, and in the case of Triton one can be plagued by variable amounts of light from the wings of the Neptune pointspread function. Since it is usually difficult to determine the precise background level throughout the entire time span of the occultation photometry, one must resort to estimating the possible time variability of the background and generate a set of inversions with different samples of background variability and evaluate the effects of these on the results.

\subsubsection{Correlated Noise}

Related to the problem of a variable background would be the effect of any correlated noise in the occultation light curve - noise that does not have a flat power spectrum, and whose values in adjacent integration intervals are correlated with one another. In addition to the variable-background example just discussed, stellar scintillation in Earth's atmosphere (Young 1967) provides another example of correlated noise. One can investigate these effects numerically by generating several random samples with the expected level of background variation and comparing the inversion results from each. The errors implied by the variation of the results would be in addition to those calculated for the Gaussian white noise described in $\S 4$.

\subsubsection{Far-Limb Flux}

Another potential source of systematic error in the inversion results is the presence of an unknown level of refracted stellar flux from the far limb. This effect is of practical concern for small bodies for two reasons: (1) the far-limb flux comes from levels not many scale heights below half-light, so it does not experience great attenuation from differential refraction and is less likely to suffer any extinction (by haze, clouds, or molecular extinction), and (2) small-body occultations are more likely observed in a mode in which the stellar fluxes from all portions of the limb are added together. Ideally, one could avoid mixing the near- and far-limb fluxes by observing an occultation with an imaging mode in which the stellar images appearing on different portions of the limb can be resolved and recorded separately.

If we consider a spherical body with the same vertical atmospheric structure everywhere, the occultation light curve will be symmetric about its midpoint. For a light curve recorded by a station passing near the center of the occultation shadow, the central flux will be composed of two nearly equal components coming from opposite limbs of the occulting body. (Precisely at the center of the shadow of a spherical body, the flux comes from the entire limb.) Without making any assumptions about the vertical atmospheric structure, however, there is no unique way to separate the near- and far-limb fluxes. Ways to cope with this issue are to (1) make some assumptions about the atmospheric structure that produces the flux from the far limb, (2) use a method of observation that excludes the far-limb flux from the record of the near-limb flux, and (3) end the inversion calculation well before the center of the light curve.

\subsection{Radial Resolution}

In our formulation of the inversion method, the fundamental limitation on the radial resolution of the temperature and other profiles derived from the inversion of the light curves is the resolution of the data, $\Delta y$, in the observer plane (Fig. 1). For a spherically symmetric atmosphere, however, any of three effects may limit the radial resolution even further: ray crossing, Fresnel diffraction, and the finite angular diameter of the occulted star. The relative importance of these depends on the particular occultation. None of these effects were included in the assumptions ( $\S 2.1$ ) underlying our formulation of the inversion method, so in this section we shall discuss how they may limit the radial resolution of the inversion profiles.

\subsubsection{Ray Crossing}

As described by Elliot \& Veverka (1976), ray crossing occurs when the rays of starlight arrive in the observer plane in a different radial order from that in which they entered the body plane (see their Fig. 7). Whether this happens depends on the relative magnitude of the derivative of the refraction angle and the distance $D$ between the body and observer planes (Fig. 1). In the equation below, we 
summarize the conditions for which ray crossing (and the associated phenomenon of perfect focusing) occurs:

$$
\begin{array}{ll}
\frac{d \theta}{d r} \geq 0, & \text { no ray crossing at any distance } ; \\
\frac{d \theta}{d r} \geq-\frac{1}{D}, & \text { no ray crossing at distance } D ; \\
\frac{d \theta}{d r}=-\frac{1}{D}, & \text { perfect focus at distance } D ; \\
\frac{d \theta}{d r}<-\frac{1}{D}, & \text { ray crossing at distance } D .
\end{array}
$$

For an isothermal atmosphere, the first condition of equation (100) holds, and ray crossing never occurs. Atmospheric dynamical phenomena-such as waves and turbulence - add additional gradients to the refractivity that tend to be a fraction of the basic exponential gradient. Although the preferred direction for most of these perturbations is parallel to the gravity gradient, they can have components perpendicular to the gravitational gradient as well. Expressed in absolute terms, these fractional perturbations of the refractivity gradient become larger deeper into the atmosphere, so that one can begin to see "spikes" of intensity (Elliot \& Veverka 1976) in occultation light curves that become progressively larger deeper into the occultation light curve. Although these spikes are common features of occultation light curves for the giant planets, they are less common in occultation light curves for Pluto (Elliot et al. 1989, 2003; Sicardy et al. 2003) and so far undetectable in occultation light curves for Triton.

In terms of our inversion method, any ray crossing that occurred within a shell, $\Delta y_{i}$ (see Fig. 2), would make no difference. However, if spikes were visible in the light curve, then one would want to use the inversion technique on the data at full resolution just to determine what atmospheric structures are implied by the spikes. Here one must exercise caution, since ray crossing could be occurring on the scale of the data resolution. Several approaches can be used for guidance. First, if one is willing to assume that the perturbations causing the spikes represent a fraction of the basic refractivity gradients, then the portion of the light curve corresponding to the atmosphere above the region of well-focused spikes should be relatively free of ray crossing, while the portion of the light curve corresponding to deeper regions of the atmosphere will very likely have ray crossing. The second approach applies to individual spikes when multiwavelength observations are available and one can examine the structure of a given spike at different wavelengths. Since the refractivity for gases generally decreases with increasing wavelength, a spike that is more focused at the blue wavelengths than the red has not yet come to a focus (considered as a function of distance from the body plane shown in Fig. 1). However, a spike that is more focused at red than at blue wavelengths has already come to a focus at a closer distance to the body plane and now exhibits ray crossing at the observer plane. Finally, a third approach (which again applies to individual spikes) would be to examine the light curve for caustic spikes (Cooray \& Elliot 2003). These indicate that ray crossing has occurred, and the width of the caustic spike tells one the extent of the ray crossing.

In summary, ray crossing sets a limit on the radial resolution, since one must average the data on a scale that includes the crossed rays in a single data interval $\Delta y$ in order to avoid errors in the inversion profiles.

\subsubsection{Fresnel Diffraction}

Another limit to the radial resolution of the inversion profiles is Fresnel diffraction, which, for monochromatic light of wavelength $\lambda$, is characterized by the scale $(\lambda D)^{1 / 2}$. This is about $1.8 \mathrm{~km}$ for an observer- to body-plane distance $D=30 \mathrm{AU}$ and a wavelength of $0.7 \mu \mathrm{m}$. Since the Fresnel scale is based on the region of phase coherence, the additional phase shift caused by the passage of the light through the atmospheric gases causes a shrinkage of the Fresnel scale by a factor of the normalized stellar flux, $\phi$, so that the Fresnel scale becomes $\phi(\lambda D)^{1 / 2}$. This means that the radial resolution for structure within the occulting atmosphere improves as the occultation probes deeper (Young 1976). Although diffraction effects are commonly observed in lunar occultations of stars (e.g., Nather \& Evans 1970) and planetary rings (e.g., Elliot et al. 1984; Roques, Moncuquet, \& Sicardy 1987), only recently have diffraction fringes been directly observed in an atmospheric occultation (Cooray \& Elliot 2003). As for ray crossing, the data must be averaged to avoid introducing erroneous structure into the inversion profiles caused by the diffraction fringes.

\subsubsection{Stellar Diameter}

A third limit to the radial resolution of inversion profiles is the angular diameter of the occulted star. The effect of finite stellar size enters as an averaging process that determines the intensity distribution in the observer plane. The intensity distribution in the observer plane for a point source is convolved with the intensity distribution of the star (Elliot, Rages, \& Veverka 1976), and for stars with particularly large angular diameters, this can be the dominant effect that limits the radial resolution of the profiles. In contrast with ray crossing and diffraction, which can produce spurious effects in the profiles if the data are not sufficiently averaged prior to inversion, the effect of the stellar diameter automatically averages the data and filters out the smaller scale structure.

For the two stars of particular interest in this paper, we find their approximate diameters, projected at the distance (Table 9 below) of the occulting body, to be $\sim 1 \mathrm{~km}$ for P8 $(V=12.4, B-V=0.7)$ and $\sim 4 \mathrm{~km}$ for $\operatorname{Tr} 180(V=10.5$, $B-V=0.8)$. Even with their estimated uncertainties of $\pm 50 \%$, these values are much smaller than a scale height. More accurate projected diameters for these stars could be determined from the relationships given by van Belle (1999) if the $K$ magnitudes and luminosity classes for P8 and Tr180 were available.

\subsection{Horizontal Atmospheric Structure}

A fundamental assumption ( $\S 2.1)$ of the inversion method is that the occulting atmosphere is spherically symmetric, which means there would be no refractivity gradients in either horizontal direction (i.e., perpendicular to the radial direction, $r$ ). Horizontal refractivity gradients along the line of sight (the $x$-direction in Fig. 1) would mean that the refraction angle determined with equation (6) would represent an average radial structure of the atmosphere in the region covered by the integration. Horizontal gradients perpendicular to the line of sight would be manifested as a difference in occultation light curves recorded at two stations in the observer plane that were displaced in this direction. Or, from a single station, one would see 
differences between the immersion and emersion portions of the occultation light curve.

Also, effects of horizontal structure could be misinterpreted as vertical structure. This arises from the fact that the stellar flux sampled at a given time is formed by the atmospheric column perpendicular to the limb, which is continuously changing if the observer's path is not perpendicular to the projection of the planetary limb into the observer plane. Since our formulation of the inversion method interprets any change in the stellar flux as a function of only the radius from the center of the shadow, any variations due to horizontal structure would be interpreted as being due to vertical structure. This aliasing of horizontal atmospheric structure as vertical structure can be avoided if the observer can choose a path that is perpendicular to the projection of the planetary limb into the observer plane - as was done to a good approximation with the KAO for the occultation of $\epsilon$ Geminorum by Mars (Elliot et al. 1977).

Horizontal atmospheric structure could arise from atmospheric waves or turbulence. For the giant planets and Titan, an extensive literature discusses both the observational (Fairall 1972; Veverka et al. 1972; Elliot \& Veverka 1976; French et al. 1982; Sicardy et al. 1999) and theoretical (Young 1976; French \& Lovelace 1983; Hubbard \& Jokipii 1977; Hubbard, Jokipii, \& Wilking 1978; Narayan \& Hubbard 1988) aspects of this issue. Fortunately for the study of Pluto's and Triton's atmospheres with stellar occultations, horizontal structure seems to be entirely absent or very weak. Possible examples of horizontal structure for Triton will be discussed below ( $\S 99$ and 10.2).

\subsection{Boundary Condition}

The specific boundary condition ( $\S 3.3$ ) used for the inversions in this paper was originally formulated (Paper I) for testing whether Pluto's occultation light curve recorded by the KAO was consistent with that produced by an isothermal atmosphere. The power-law thermal profile given by the equation (59) is not a physical model but was chosen for its analytic convenience. The rationale was that if Pluto's occultation light curve were consistent with an isothermal atmosphere, then the form of the model used to introduce a thermal gradient (to first order) would not really matter. On the other hand, if a thermal gradient were found to be present, then one should be concerned about finding an appropriate physical model for the atmosphere. Since the results of the model fit to the KAO light curve (above the kink) showed that it was consistent with its being produced by an isothermal atmosphere, the model of Paper I fulfilled its original purpose.

The availability of this model and our extensive experience with it led us to use it for the boundary condition for the inversions in this paper. However, a potential user should be aware of its shortcomings as a general boundary condition for inversions. The first and foremost of these is that it is not a physical model. Second, it does not include the mass of the atmosphere in its formulation, which can lead to infinities at large radii. Examining equation (69), for example, we see that $d \theta_{b}(r)$ diverges for large $r$ when $b \leq-1$ (although a value for $b \leq-1$ may define the light curve very well near half-light). Third, the asymptotic series (Acton 1970) in equations (64) and (67) provide a progressively worse approximation as the value of $b$ increases. This is evident in the test cases for nonisothermal atmospheres (§ 6.2.3).

A boundary condition for inversions that would eliminate the shortcomings of the one we have used in this paper would be based on the numerical integration of an appropriate physical model (e.g., Strobel et al. 1996) for the atmosphere of the occulting body. One can then adjust certain atmospheric parameters of the physical model by leastsquares fitting of the light curve above the boundary flux with the numerical method described by Chamberlain \& Elliot (1997). Two of these fitted parameters (or a combination of them) could correspond to the half-light radius and the "scale height" at half-light, respectively. For highquality data, one could fit a third parameter that would correspond to a "scale height gradient."

Judicious choice of boundary condition is important because the kernels for the refractivity and pressure integrals in equations (35) and (36) have significant values for many scale heights above the inversion region (see Fig. 3 of French et al. 1978). The sensitivity of the inversion profiles to different boundary condition parameters was explored in $\S 6.2 .3$, but we have not explored the sensitivity of the inversion to different types of boundary conditions.

\subsection{Inversion of Large-Body Light Curves}

Although our inversion method has been formulated for small-body occultation light curves, it can be used for large bodies as well. A large body typically rotates fast enough to invalidate two assumptions used in our method, so for large bodies we must consider that (1) the body's equilibrium shape is not spherical, and (2) hydrostatic equilibrium is determined not only by the gravitational force, but by centrifugal force as well (which adds a term proportional to radius; see Paper I). Inversion of large-body occultation light curves can be carried out with classical methods (see, e.g., French et al. 1978). However, if one desires to use identical code for the inversion of large- and small-body light curves, the equations presented here can be adapted to large bodies. The new assumption made is that the local acceleration (having contributions from both gravity and centrifugal force) is constant throughout the boundary and inversion region. Hence the "radius"-which appears as a single symbol, $r$, in the small-body formulation-has three different uses in the large-body formulation. The first is a radial coordinate with an origin at the center of the body. The second is the radius of curvature of the elliptical figure of the body along the line of sight from the observer to the occulted star, which is the relevant radius for the differential refraction perpendicular to the density gradient in the atmosphere (Hilbert 2001, p. 41). The third is the radius of curvature in the plane perpendicular to this line-of-sight direction, which controls the lateral focusing (Hilbert 2001, p. 41).

To apply the equations presented here to the inversion of a large-body light curve, one first determines the boundary radius, $r_{b}$, in the body's atmosphere and calculates the radius of curvature of the atmosphere, $r_{c}$, along the line of sight at $r=r_{b}$. Then one replaces $r$ by an effective radius, $r_{\text {eff }}=r-r_{b}+r_{c}$, in equations (43) and (44). Finally, the mass, $M_{p}$, of the occulting body is replaced (in eq. [21] and thereafter) by an effective mass, $M_{\text {eff, }}$, such that the local acceleration due to the combination of gravity and 
centrifugal force equals the local acceleration that would be calculated for gravity alone with $M_{\text {eff. }}$. For large-body occultations, the effect of lateral focusing is negligible and can be ignored.

\section{APPLICATION TO THE 1988 PLUTO OCCULTATION}

The 1988 June 9 stellar occultation of the star P8 by Pluto (Mink \& Klemola 1985) was extensively observed from sites in the southwestern Pacific, including the KAO, Australia, and New Zealand (Hubbard et al. 1988; Elliot et al. 1989; Millis et al. 1993). The highest quality data were recorded from the KAO, and these are displayed in Figure 12 (Elliot et al. 1989). The light curve closely matches an isothermal atmospheric model above half-light, but below half-light it decreases more precipitously than would be expected for an isothermal atmosphere. This characteristic has been explained by two different inferences about Pluto's atmospheric structure: (1) an extinction layer (Elliot et al. 1989; Paper I) and (2) a steep thermal gradient (Eshleman 1989; Hubbard et al. 1990b). The steep-thermal-gradient model assumes negligible extinction, so we can determine the thermal profile in Pluto's atmosphere consistently with this view through the use of the inversion method developed here. If this atmosphere has no extinction, then this light curve certainly qualifies for inversion according to our criterion discussed in $\S 7.2$ - as can be seen by its deviation from an isothermal model (see Fig. 3 in Elliot et al. 1989).

Carrying out the inversion requires a calibrated light curve, which means that we need to accurately know the level for zero flux from the star. A predetermined zero level was not necessary for the earlier atmospheric model-fitting analysis of the occultation light curve, since the zero level was a well-determined parameter from the model fit (Elliot et al. 1989; Paper I). One method for establishing the zero level of the light curve is to accurately determine the ratio of light from Pluto to that of $\mathrm{P} 8$ just prior to the occultation. Accordingly, a few hours before the occultation (when the images of Pluto and P8 were well separated), several series of frames containing the images of Pluto and P8 were recorded aboard the KAO with SNAPSHOT (the instrument used to record the occultation light curve as well; Dunham et al. 1985). Exposure times of 10, 15, and $30 \mathrm{~s}$ were

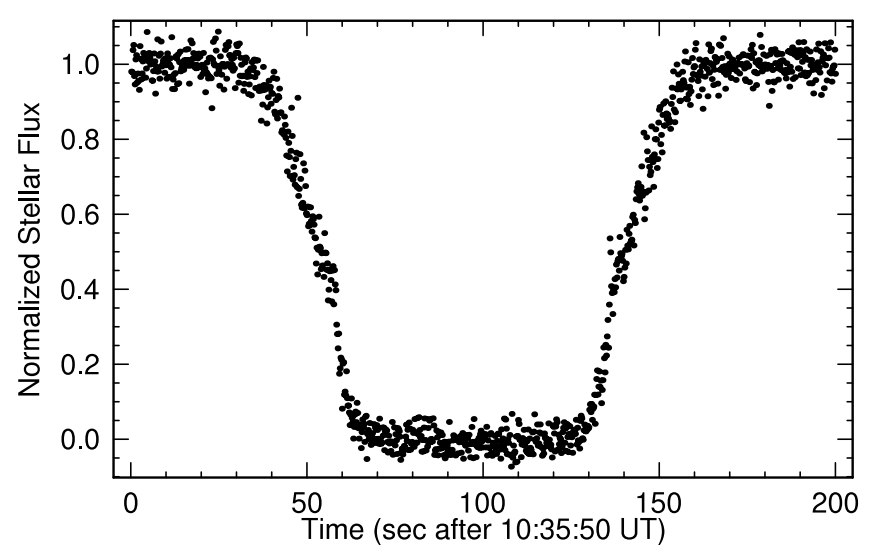

FIG. 12.-Pluto occultation. The KAO light curve for Pluto's occultation of the star P8 (Mink \& Klemola 1985), which occurred on 1988 June 9, is plotted for a Pluto-to-star signal ratio of 0.24 . Each point represents a $0.2 \mathrm{~s}$ integration (adapted from Elliot et al. 1989). used. The image of Charon was effectively merged with that of Pluto, since the Pluto-Charon separation was much less than an arcsecond at the time, and the KAO images had a full width at half-maximum (FWHM) of $4^{\prime \prime}-5^{\prime \prime}$. Hence we have treated these bodies as if they were a single object, referred to here as "Pluto."

To establish the desired signal ratio, we fitted the Pluto and P8 images simultaneously with a generalized Lorentzian point-spread function (PSF) model described by Bosh et al. (1992). Each dual-image fit had seven free parameters: background, the centers of the two objects, the peak signal from P8, and the ratio of the signal from Pluto to that of P8. The signal ratios from these fits are displayed in Table 6, along with the frame number, start time, exposure time, and rms residual for each fit. The ratios all lie within the range from $0.2294 \pm 0.0035$ to $0.2437 \pm 0.0016$. We give the mean ratio from all the frames in the first row of Table 7, followed by the ratios for each set of exposure times considered separately. The last row of Table 7 has the signal ratio of Pluto to P8 calculated from the background and star levels determined in the atmospheric model fit contained in Table 9 of Paper I. The background level in the occultation light curve refers to the signal from Pluto alone, since other backgrounds from the sky and detector were removed in the construction of the occultation light curve.

The signal ratios for the 10 and $15 \mathrm{~s}$ exposures are remarkably consistent with each other, with differences consistent within the standard error of their respective sample means. Also, the standard deviations of the 10 and $15 \mathrm{~s}$ exposures are consistent with the formal errors from the PSF fits (Table 7). The mean ratios obtained for the "occultation model" (Paper I) and those from the 10 and $15 \mathrm{~s}$ exposures agree within their formal errors, while the ratio from the $30 \mathrm{~s}$ exposures is somewhat higher. One would then tend to believe that the ratio lies near the three values that agree, but we have no explanation for the higher value for the $30 \mathrm{~s}$ exposures. We shall not pursue this issue any further here. Rather, we shall carry out a series of inversions on light curves constructed for a set of three different Pluto/P8 signal ratios that bracket the possibilities: $0.23,0.24$, and 0.25 . Comparison of these results will show us the effect in the resulting thermal profiles of the remaining uncertainty in the zero level of the occultation light curve.

We carried out inversions for the immersion and emersion sections of the light curve displayed in Figure 12 for the above three choices for the Pluto/P8 ratio. For each inversion, we fitted the boundary model (with thermal gradient exponent as a free parameter) down to a flux level of approximately 0.6 , and the results of the boundary fits for a Pluto/P8 signal ratio of 0.24 are given in Table 8 . The parameters used to carry out the inversions are given in Table 9, and the values for the physical constants used are given in Table 10. Below the boundary fit, we averaged the radii within Pluto's atmosphere to a minimum value of 1.0 $\mathrm{km}$. At the beginning of the inversion, these averages were nearly $2 \mathrm{~km}$ because the time resolution of the data was only $0.2 \mathrm{~s}$. The radii, refractivities, number densities, pressures, temperatures, and scale heights from the inversions with a Pluto/P8 signal ratio of 0.24 are given in Table 11 (immersion) and Table 12 (emersion). In these tables the errors given on the radii are those arising from the errors in the fluxes, but these are dominated by a systematic error that arises from the imprecisely known position of the KAO 
TABLE 6

Signal Ratios: Pluto to Star

\begin{tabular}{|c|c|c|c|c|}
\hline Frame & $\begin{array}{l}\text { Start Time }^{\mathrm{a}} \\
\text { (UT) }\end{array}$ & $\begin{array}{l}\text { Exposure Time } \\
\text { (s) }\end{array}$ & $\begin{array}{l}\text { Signal Ratio } \\
\text { (Pluto/Star) }\end{array}$ & $\begin{array}{c}\text { rms Residual }^{\mathrm{b}} \\
\text { (DN) }\end{array}$ \\
\hline $1 \ldots \ldots$ & $06: 28: 53$ & 30 & $0.2425 \pm 0.0016$ & 11.4 \\
\hline 2. & 06:30:02 & 30 & $0.2431 \pm 0.0021$ & 15.3 \\
\hline$\ldots \ldots \ldots$ & $06: 31: 56$ & 30 & $0.2424 \pm 0.0035$ & 20.3 \\
\hline $6 \ldots \ldots \ldots \ldots \ldots \ldots \ldots \ldots \ldots \ldots \ldots \ldots$ & $06: 33: 50$ & 30 & $0.2435 \pm 0.0023$ & 15.9 \\
\hline 7 . & $06: 34: 47$ & 30 & $0.2421 \pm 0.0024$ & 16.6 \\
\hline $8 \ldots \ldots \ldots \ldots \ldots$ & $06: 35: 44$ & 30 & $0.2420 \pm 0.0031$ & 21.5 \\
\hline 9.1. & $06: 36: 41$ & 30 & $0.2408 \pm 0.0033$ & 22.6 \\
\hline $10 \ldots \ldots \ldots \ldots \ldots \ldots$ & $06: 37: 38$ & 30 & $0.2406 \pm 0.0032$ & 23.2 \\
\hline $11 \ldots \ldots \ldots \ldots \ldots$ & $06: 38: 35$ & 30 & $0.2421 \pm 0.0025$ & 19.0 \\
\hline $12 .$. & $06: 39: 32$ & 30 & $0.2437 \pm 0.0028$ & 19.7 \\
\hline $13 \ldots$ & $06: 40: 31$ & 30 & $0.2415 \pm 0.0024$ & 14.5 \\
\hline $14 \ldots \ldots \ldots \ldots \ldots$ & $06: 41: 30$ & 30 & $0.2428 \pm 0.0023$ & 15.6 \\
\hline $15 \ldots \ldots \ldots \ldots \ldots \ldots$ & $06: 42: 27$ & 30 & $0.2376 \pm 0.0023$ & 14.4 \\
\hline $19 \ldots \ldots \ldots \ldots \ldots \ldots$ & $06: 46: 17$ & 30 & $0.2432 \pm 0.0035$ & 24.7 \\
\hline $20 \ldots \ldots \ldots \ldots \ldots \ldots$ & $06: 47: 15$ & 30 & $0.2419 \pm 0.0040$ & 26.8 \\
\hline $21 \ldots \ldots \ldots \ldots \ldots \ldots$ & $06: 48: 12$ & 30 & $0.2295 \pm 0.0035$ & 17.3 \\
\hline .............. & $06: 49: 11$ & 30 & $0.2416 \pm 0.0031$ & 20.9 \\
\hline $23 \ldots \ldots \ldots \ldots$ & 06:54:02 & 10 & $0.2339 \pm 0.0019$ & 7.5 \\
\hline ……...... & $06: 55: 47$ & 10 & $0.2305 \pm 0.0040$ & 14.6 \\
\hline $25 \ldots \ldots \ldots \ldots \ldots \ldots$ & $06: 56: 49$ & 10 & $0.2294 \pm 0.0035$ & 12.3 \\
\hline $26 \ldots \ldots \ldots \ldots \ldots \ldots$ & $06: 57: 48$ & 10 & $0.2300 \pm 0.0029$ & 9.1 \\
\hline $27 \ldots \ldots \ldots \ldots \ldots$ & $06: 58: 47$ & 15 & $0.2322 \pm 0.0025$ & 11.4 \\
\hline $28 \ldots \ldots \ldots \ldots \ldots \ldots$ & $06: 59: 43$ & 15 & $0.2318 \pm 0.0025$ & 14.1 \\
\hline $29 \ldots \ldots \ldots \ldots \ldots \ldots$ & $07: 00: 26$ & 15 & $0.2333 \pm 0.0024$ & 13.7 \\
\hline $30 \ldots \ldots \ldots \ldots \ldots \ldots$ & 07:01:08 & 15 & $0.2317 \pm 0.0026$ & 16.5 \\
\hline $31 \ldots \ldots \ldots \ldots \ldots \ldots$ & 07:01:50 & 15 & $0.2299 \pm 0.0030$ & 17.6 \\
\hline$\ldots \ldots \ldots \ldots$ & $07: 02: 32$ & 15 & $0.2324 \pm 0.0026$ & 15.7 \\
\hline $33 \ldots \ldots \ldots \ldots \ldots \ldots$ & 07:03:15 & 15 & $0.2294 \pm 0.0039$ & 21.0 \\
\hline $34 \ldots \ldots \ldots \ldots \ldots \ldots \ldots$ & 07:03:57 & 15 & $0.2341 \pm 0.0016$ & 9.9 \\
\hline $35 \ldots \ldots \ldots$ & 07:04:39 & 15 & $0.2314 \pm 0.0022$ & 14.1 \\
\hline $36 \ldots \ldots$ & 07:05:21 & 15 & $0.2312 \pm 0.0026$ & 15.8 \\
\hline $37 \ldots \ldots \ldots \ldots \ldots \ldots$ & 07:06:04 & 15 & $0.2321 \pm 0.0024$ & 15.1 \\
\hline $38 \ldots \ldots \ldots \ldots \ldots \ldots$ & 07:06:46 & 15 & $0.2327 \pm 0.0023$ & 14.2 \\
\hline $39 \ldots \ldots \ldots \ldots \ldots$ & 07:07:29 & 15 & $0.2312 \pm 0.0022$ & 13.7 \\
\hline $40 \ldots \ldots \ldots \ldots \ldots \ldots$ & 07:08:11 & 15 & $0.2306 \pm 0.0030$ & 18.3 \\
\hline $41 \ldots \ldots \ldots \ldots \ldots \ldots$ & 07:08:53 & 15 & $0.2308 \pm 0.0025$ & 15.6 \\
\hline $42 \ldots \ldots \ldots \ldots \ldots \ldots$ & 07:09:35 & 15 & $0.2300 \pm 0.0033$ & 19.2 \\
\hline
\end{tabular}

a UT start times are on 1988 June 9.

$\mathrm{b}$ These rms residuals per pixel in data numbers (DN) are displayed to illustrate the relative quality of the least-squares fit results for each frame. As an example of a complete set of fitted parameters, for frame 1 the fitted background level was $151.8 \pm 0.3 \mathrm{DN}_{\text {pixel }}{ }^{-1}$, the peak signal from P8 was $1936 \pm 6 \mathrm{DN}$, its row and column centers were $288.212 \pm 0.005$ and $259.104 \pm 0.005$ pixels, the signal ratio is given in the previous column of this table, the row and column offsets of Pluto from P8 were $7.718 \pm 0.019$ and $-9.310 \pm 0.019$ pixels, the diameter of the (forced) common PSF was $4.976 \pm 0.013$ pixels, and the "shape index" of the PSF (Bosh et al. 1992) was $3.384 \pm 0.014$. The focal-plane scale was 1"'1 pixel $^{-1}$ (Elliot et al. 1989).

TABLE 7

Summary of Pluto-to-Star Signal Ratios

\begin{tabular}{cccc}
\hline \hline Sample & $\begin{array}{c}\text { Mean } \\
\text { Ratio }\end{array}$ & $\begin{array}{c}\text { Standard } \\
\text { Deviation }\end{array}$ & $\begin{array}{c}\text { Standard Error of } \\
\text { Sample Mean }\end{array}$ \\
\hline All exposures ................ & 0.2359 & 0.0055 & 0.0009 \\
10 s exposures ............. & 0.2309 & 0.0020 & 0.0010 \\
15 s exposures ............ & 0.2315 & 0.0013 & 0.0003 \\
30 s exposures ............ & 0.2412 & 0.0034 & 0.0008 \\
Occultation model $^{\mathrm{a}} \ldots . .$. & 0.2335 & $\ldots$ & $0.0026^{\mathrm{b}}$ \\
\hline
\end{tabular}

a Calculated from " background level" and "star level" in Table 9 of Paper I.

b Calculated from the errors in "background level" and "star level" in Table 9 of Paper I.
(Elliot et al. 1989; Millis et al. 1993). This issue will be discussed at the end of this section.

To illustrate the systematic effects that arise from choosing different signal ratios, the temperature profiles for the inversions for all three signal ratios are displayed in Figure 13, where the immersion profiles are displayed in the top panel and the emersion profiles in the bottom panel. For clarity, the individual points and their error bars are not shown, but the points have been connected with lines. All temperature profiles show a nearly isothermal section at higher radii, with an abrupt drop in temperature beginning at a radius of about $1212-1214 \mathrm{~km}$. The effect of different assumed signal ratios is to displace the temperature profiles to larger radii and lower temperatures as the ratio increases. 
TABLE 8

Pluto and Triton Boundary Fit Information

\begin{tabular}{|c|c|c|c|c|}
\hline \multirow[b]{2}{*}{ Parameter } & \multicolumn{2}{|c|}{ Pluto } & \multicolumn{2}{|c|}{ TRITON } \\
\hline & Immersion & Emersion & Immersion & Emersion \\
\hline Background ... & 0 & 0 & 0 & 0 \\
\hline Slope $\left(10^{-7} \mathrm{~s}^{-1}\right) \ldots \ldots \ldots \ldots \ldots \ldots$ & 0 & 0 & $-3.9 \pm 1.9$ & $15.1 \pm 1.6$ \\
\hline Star plus background.. & 1 & 1 & $0.9967 \pm 0.0003$ & $0.9956 \pm 0.0003$ \\
\hline Half-light radius, $r_{h}(\mathrm{~km}) \ldots \ldots \ldots \ldots \ldots \ldots \ldots \ldots \ldots \ldots \ldots$ & $1234 \pm 5$ & $1233 \pm 5$ & $1450.3 \pm 0.3$ & $1447.6 \pm 0.3$ \\
\hline 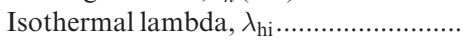 & $19.9 \pm 2.3$ & $19.4 \pm 2.2$ & $66.3 \pm 1.4$ & $66.2 \pm 1.3$ \\
\hline Thermal gradient exponent, $b \ldots \ldots \ldots \ldots \ldots . .$. & $-1.6 \pm 1.5$ & $-1.1 \pm 1.5$ & $6.4 \pm 1.2$ & $5.2 \pm 1.1$ \\
\hline 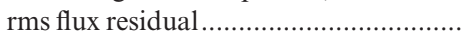 & 0.035 & 0.034 & 0.0023 & 0.0024 \\
\hline Signal-to-noise ratio, ${ }^{\mathrm{a}}(\mathrm{S} / \mathrm{N})_{H} \ldots \ldots \ldots \ldots \ldots$ & 125 & 128 & 608 & 761 \\
\hline
\end{tabular}

a The signal-to-noise ratio per scale height $\left[(\mathrm{S} / \mathrm{N})_{H}\right]$ was determined from the rms residuals in the boundary fit for the first 125 points in each light curve. These numbers are directly comparable to the test cases in Fig. 9 . The difference between $(\mathrm{S} / \mathrm{N})_{H}$ for Triton immersion and emersion arises from the large change in Triton's shadow speed relative to HST during the course of the event.

This displacement is small, however, relative to the errors in the temperature profiles, as we can conclude from the thermal profile plots in Figure 14. Here the top panel shows the thermal profiles with error bars for immersion and emersion (both for a Pluto/P8 signal ratio of 0.24 ), and the bottom panel shows the same profiles without error bars. We note that any differences between the immersion and emersion profiles lie within the error bars, and that a systematic displacement due to any reasonable choice of signal ratio (Fig. 13) would be much smaller than the error bars. Also, we reiterate that each error bar indicates the rms error in the temperature, but adjacent points have highly correlated errors, so that local temperature differences have much smaller errors than one would visually estimate. We shall return to this point later.

We also inverted the Pluto occultation curve with an isothermal boundary condition, since the thermal profile is close to isothermal in Figure 14 and some theoretical models (Yelle \& Lunine 1989; Hubbard et al. 1990b; Lellouch 1994; Strobel et al. 1996; Krasnopolsky \& Cruikshank 1999) predict a nearly isothermal profile in this region. Figure 15 shows the inversion for three different Pluto/P8 signal ratios, which shows a similar pattern to the inversion with a boundary condition that allowed a thermal gradient (Fig. 13). Figure 16 shows the inversion with error bars (top) for the immersion and emersion light curves with a Pluto/ P8 signal ratio of 0.24 . Note that the errors for an isothermal boundary condition are about half those for the boundary condition that allowed a thermal gradient (Fig. 14). The bottom panel of Figure 16 shows the temperature profiles plotted without error bars.

TABLE 9

Pluto and Triton InVersion Parameters

\begin{tabular}{|c|c|c|}
\hline Parameter & Pluto & Triton \\
\hline Distance $\left(10^{9} \mathrm{~km}\right)$. & 4.323 & 4.54467 \\
\hline Gas ..................... & $\mathrm{N}_{2}$ & $\mathrm{~N}_{2}$ \\
\hline Mean molecular weight (amu) ................ & 28.01 & 28.01 \\
\hline Refractivity $\left(10^{-4}\right) \ldots \ldots \ldots \ldots \ldots \ldots$ & 2.98 & 2.98 \\
\hline Mass $\left(10^{22} \mathrm{~kg}\right) \ldots \ldots \ldots$ & 1.312 & 2.13975 \\
\hline Integration upper limit $(\mathrm{km}) \ldots \ldots \ldots \ldots \ldots \ldots$ & 10,000 & 10,000 \\
\hline Series order in eqs. (64) and (67). & 2 & 2 \\
\hline Radial resolution, $\Delta y(\mathrm{~km}) \ldots \ldots \ldots \ldots \ldots \ldots$ & $\leq 3.7$ & $\leq 12.0$ \\
\hline Shell thickness, $\Delta r(\mathrm{~km}) \ldots \ldots \ldots$. & $\geq 1.0$ & $\geq 1.0$ \\
\hline Flux level at boundary ......................... & $\sim 0.5$ & $\sim 0.5$ \\
\hline
\end{tabular}

In order to understand where the errors arise in the inversion process, it is instructive to display the thermal profiles with the error contributions from the boundary fit separately from the error contributions from the inversion summation. This is done in Figure 17, where we see that the summation error remains substantially smaller than the boundary error. This dominance of the error from the boundary fit agrees with that found in our test cases (Fig. 10), since the Pluto inversion ends less than 1 scale height $(\sim 55 \mathrm{~km})$ below half-light.

An important feature of the inversion profiles that bears on our understanding of the processes occurring in Pluto's atmosphere is the thermal gradient at the lowest altitude. Looking at the error bars on the temperatures in the lower part of the thermal profiles (Figs. 14 and 16), one might conclude that the gradient is hardly significant. However, errors in adjacent temperatures are highly correlated, so the error in their difference is much smaller than one would surmise from the error in the individual temperatures. Calculation of the correlation coefficients needed to find the error in the temperature differences is beyond the scope of this work, but we can get a rough idea of the error in the gradient from the difference between the immersion and emersion gradients. In Table 11, the difference between the radius of the last two entries is $1.1 \mathrm{~km}$. Their difference in temperature is $5 \mathrm{~K}$, which yields a gradient of $4.5 \mathrm{~K} \mathrm{~km}^{-1}$ (for a radius of $\sim 1206 \mathrm{~km}$ ). The same calculation for the last two entries in Table 12 for the emersion inversion yields a gradient of 3.3 $\mathrm{K} \mathrm{km}^{-1}$. For our later discussions we shall use the approximate average value of $3.9 \mathrm{~K} \mathrm{~km}^{-1}$, realizing that the error in this value is likely about $10 \%-20 \%$. To be definite, we use the formal rms error for the average of two values so that our final result for the gradient is $3.9 \pm 0.6 \mathrm{~K} \mathrm{~km}^{-1}$. This gradient corresponds to an atmospheric temperature of $\sim 93$ $\mathrm{K}$. Of course, the validity of this thermal gradient depends on the assumption of that extinction effects from Pluto's

TABLE 10

Physical Constants

\begin{tabular}{|c|c|}
\hline Constant & Value \\
\hline Gravitational constant, $G\left(\mathrm{~kg}^{-1} \mathrm{~m}^{3} \mathrm{~s}^{-2}\right)$. & $6.67320 \times 10^{-11}$ \\
\hline 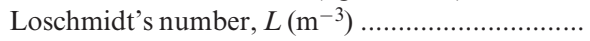 & $2.68684 \times 10^{24}$ \\
\hline Boltzmann's constant, $k\left(\mathrm{~kg} \mathrm{~m}^{2} \mathrm{~s}^{-2} \mathrm{~K}^{-1}\right) \ldots \ldots \ldots \ldots \ldots$ & $1.38062 \times 10^{-23}$ \\
\hline 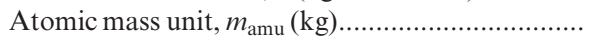 & $1.66030 \times 10^{-27}$ \\
\hline
\end{tabular}


TABLE 11

Pluto INVERSION: IMMERSion

\begin{tabular}{|c|c|c|c|c|c|c|c|}
\hline $\begin{array}{l}\text { Observer Center } \\
\text { Distance, } y \\
(\mathrm{~km})\end{array}$ & $\begin{array}{c}\text { Stellar Flux } \\
\text { (Normalized) }\end{array}$ & $\begin{array}{c}\text { Radius }^{\mathrm{a}} \\
(\mathrm{km})\end{array}$ & $\begin{array}{l}\text { Refractivity } \\
\qquad\left(10^{-9}\right)\end{array}$ & $\begin{array}{l}\text { Number Density } \\
\qquad\left(10^{14} \mathrm{~cm}^{-3}\right)\end{array}$ & $\begin{array}{l}\text { Pressure } \\
(\mu \text { bar })\end{array}$ & $\begin{array}{c}\text { Temperature } \\
\text { (K) }\end{array}$ & $\begin{array}{l}\text { Scale Height } \\
\quad(\mathrm{km})\end{array}$ \\
\hline 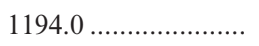 & $\mathrm{b}$ & $1245.2 \pm 1.6$ & $1.00 \pm 0.06$ & $0.91 \pm 0.05$ & $1.23 \pm 0.19$ & $98 \pm 9$ & $52 \pm 5$ \\
\hline $1191.4 \ldots \ldots \ldots \ldots \ldots \ldots \ldots$ & $0.47 \pm 0.04$ & $1244.1 \pm 1.6$ & $1.03 \pm 0.06$ & $0.93 \pm 0.05$ & $1.26 \pm 0.19$ & $98 \pm 9$ & $52 \pm 5$ \\
\hline $1188.9 \ldots \ldots \ldots \ldots \ldots \ldots \ldots$ & $0.51 \pm 0.04$ & $1242.8 \pm 1.6$ & $1.05 \pm 0.06$ & $0.95 \pm 0.05$ & $1.29 \pm 0.19$ & $99 \pm 9$ & $52 \pm 5$ \\
\hline $1186.4 \ldots \ldots \ldots \ldots \ldots \ldots \ldots$ & $0.44 \pm 0.04$ & $1241.8 \pm 1.6$ & $1.07 \pm 0.06$ & $0.97 \pm 0.05$ & $1.32 \pm 0.19$ & $99 \pm 9$ & $52 \pm 5$ \\
\hline $1183.9 \ldots \ldots \ldots \ldots \ldots \ldots$ & $0.51 \pm 0.04$ & $1240.5 \pm 1.6$ & $1.10 \pm 0.06$ & $0.99 \pm 0.05$ & $1.35 \pm 0.19$ & $99 \pm 9$ & $51 \pm 5$ \\
\hline $1181.3 \ldots \ldots$ & $0.59 \pm 0.04$ & $1239.1 \pm 1.6$ & $1.13 \pm 0.06$ & $1.02 \pm 0.05$ & $1.39 \pm 0.19$ & $99 \pm 9$ & $51 \pm 5$ \\
\hline $1178.8 \ldots \ldots$ & $0.51 \pm 0.04$ & $1237.9 \pm 1.6$ & $1.15 \pm 0.06$ & $1.04 \pm 0.05$ & $1.42 \pm 0.20$ & $99 \pm 9$ & $51 \pm 5$ \\
\hline $1176.3 \ldots \ldots \ldots \ldots \ldots \ldots \ldots$ & $0.51 \pm 0.04$ & $1236.7 \pm 1.6$ & $1.18 \pm 0.06$ & $1.06 \pm 0.05$ & $1.45 \pm 0.20$ & $99 \pm 9$ & $51 \pm 5$ \\
\hline $1173.8 \ldots \ldots \ldots \ldots \ldots \ldots \ldots$ & $0.45 \pm 0.04$ & $1235.6 \pm 1.6$ & $1.20 \pm 0.06$ & $1.08 \pm 0.05$ & $1.48 \pm 0.20$ & $99 \pm 8$ & $51 \pm 4$ \\
\hline $1171.3 \ldots \ldots \ldots \ldots \ldots \ldots$ & $0.50 \pm 0.04$ & $1234.4 \pm 1.6$ & $1.23 \pm 0.06$ & $1.11 \pm 0.05$ & $1.52 \pm 0.20$ & $99 \pm 8$ & $51 \pm 4$ \\
\hline $1168.8 \ldots \ldots \ldots \ldots \ldots \ldots$ & $0.46 \pm 0.04$ & $1233.3 \pm 1.6$ & $1.25 \pm 0.06$ & $1.13 \pm 0.05$ & $1.55 \pm 0.20$ & $99 \pm 8$ & $51 \pm 4$ \\
\hline $1166.4 \ldots \ldots \ldots \ldots \ldots \ldots \ldots$ & $0.50 \pm 0.04$ & $1232.2 \pm 1.6$ & $1.28 \pm 0.06$ & $1.16 \pm 0.05$ & $1.59 \pm 0.20$ & $99 \pm 8$ & $51 \pm 4$ \\
\hline 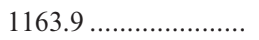 & $0.43 \pm 0.04$ & $1231.2 \pm 1.6$ & $1.31 \pm 0.06$ & $1.18 \pm 0.05$ & $1.62 \pm 0.20$ & $99 \pm 8$ & $51 \pm 4$ \\
\hline $1161.4 \ldots \ldots \ldots \ldots \ldots \ldots$ & $0.55 \pm 0.04$ & $1229.9 \pm 1.6$ & $1.34 \pm 0.06$ & $1.21 \pm 0.05$ & $1.66 \pm 0.21$ & $100 \pm 8$ & $51 \pm 4$ \\
\hline $1159.0 \ldots \ldots \ldots \ldots \ldots \ldots \ldots$ & $0.50 \pm 0.04$ & $1228.7 \pm 1.6$ & $1.37 \pm 0.06$ & $1.23 \pm 0.05$ & $1.70 \pm 0.21$ & $100 \pm 8$ & $51 \pm 4$ \\
\hline $1156.5 \ldots \ldots \ldots \ldots \ldots \ldots$ & $0.50 \pm 0.04$ & $1227.6 \pm 1.6$ & $1.39 \pm 0.06$ & $1.26 \pm 0.05$ & $1.74 \pm 0.21$ & $100 \pm 8$ & $51 \pm 4$ \\
\hline $1154.1 \ldots \ldots$. & $0.45 \pm 0.04$ & $1226.5 \pm 1.7$ & $1.42 \pm 0.06$ & $1.28 \pm 0.06$ & $1.77 \pm 0.21$ & $100 \pm 8$ & $51 \pm 4$ \\
\hline $1151.6 \ldots \ldots$. & $0.47 \pm 0.04$ & $1225.5 \pm 1.7$ & $1.45 \pm 0.06$ & $1.31 \pm 0.06$ & $1.81 \pm 0.21$ & $100 \pm 8$ & $51 \pm 4$ \\
\hline 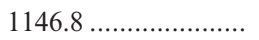 & $0.38 \pm 0.03$ & $1223.7 \pm 1.7$ & $1.50 \pm 0.06$ & $1.35 \pm 0.06$ & $1.87 \pm 0.21$ & $100 \pm 7$ & $51 \pm 4$ \\
\hline $1144.3 \ldots \ldots \ldots \ldots \ldots \ldots \ldots$ & $0.45 \pm 0.04$ & $1222.7 \pm 1.7$ & $1.53 \pm 0.06$ & $1.38 \pm 0.06$ & $1.91 \pm 0.22$ & $100 \pm 7$ & $51 \pm 4$ \\
\hline 1141.9 & $0.45 \pm 0.04$ & $1221.7 \pm 1.7$ & $1.56 \pm 0.06$ & $1.40 \pm 0.06$ & $1.95 \pm 0.22$ & $101 \pm 7$ & $51 \pm 4$ \\
\hline $1137.1 \ldots \ldots \ldots \ldots \ldots \ldots . .$. & $0.40 \pm 0.03$ & $1219.9 \pm 1.7$ & $1.61 \pm 0.06$ & $1.45 \pm 0.06$ & $2.02 \pm 0.22$ & $101 \pm 7$ & $51 \pm 4$ \\
\hline $1134.7 \ldots \ldots \ldots \ldots \ldots \ldots$ & $0.45 \pm 0.04$ & $1218.9 \pm 1.7$ & $1.64 \pm 0.06$ & $1.48 \pm 0.06$ & $2.06 \pm 0.22$ & $101 \pm 7$ & $51 \pm 4$ \\
\hline $1132.3 \ldots \ldots \ldots \ldots \ldots \ldots \ldots$ & $0.46 \pm 0.04$ & $1217.9 \pm 1.7$ & $1.67 \pm 0.06$ & $1.51 \pm 0.06$ & $2.10 \pm 0.22$ & $101 \pm 7$ & $51 \pm 4$ \\
\hline $1127.6 \ldots \ldots \ldots \ldots \ldots \ldots \ldots \ldots$ & $0.41 \pm 0.03$ & $1216.1 \pm 1.7$ & $1.73 \pm 0.06$ & $1.56 \pm 0.06$ & $2.18 \pm 0.22$ & $101 \pm 7$ & $51 \pm 4$ \\
\hline $1122.9 \ldots \ldots \ldots \ldots \ldots \ldots$ & $0.41 \pm 0.03$ & $1214.3 \pm 1.7$ & $1.79 \pm 0.06$ & $1.61 \pm 0.06$ & $2.25 \pm 0.23$ & $101 \pm 7$ & $51 \pm 3$ \\
\hline $1118.2 \ldots \ldots \ldots \ldots \ldots \ldots \ldots$ & $0.29 \pm 0.03$ & $1213.0 \pm 1.7$ & $1.83 \pm 0.06$ & $1.65 \pm 0.06$ & $2.31 \pm 0.23$ & $101 \pm 7$ & $51 \pm 3$ \\
\hline $1113.5 \ldots \ldots \ldots \ldots \ldots \ldots \ldots$ & $0.26 \pm 0.03$ & $1211.9 \pm 1.7$ & $1.88 \pm 0.06$ & $1.69 \pm 0.06$ & $2.36 \pm 0.23$ & $101 \pm 7$ & $50 \pm 3$ \\
\hline $1106.6 \ldots \ldots \ldots \ldots \ldots \ldots$ & $0.19 \pm 0.02$ & $1210.7 \pm 1.7$ & $1.93 \pm 0.06$ & $1.74 \pm 0.06$ & $2.42 \pm 0.23$ & $101 \pm 6$ & $50 \pm 3$ \\
\hline 1099.7 .......................... & $0.17 \pm 0.02$ & $1209.6 \pm 1.7$ & $1.99 \pm 0.06$ & $1.79 \pm 0.06$ & $2.47 \pm 0.23$ & $100 \pm 6$ & $50 \pm 3$ \\
\hline 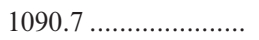 & $0.14 \pm 0.02$ & $1208.5 \pm 1.7$ & $2.06 \pm 0.06$ & $1.85 \pm 0.06$ & $2.53 \pm 0.24$ & $99 \pm 6$ & $49 \pm 3$ \\
\hline $1079.5 \ldots \ldots \ldots \ldots \ldots \ldots \ldots$ & $0.11 \pm 0.02$ & $1207.4 \pm 1.7$ & $2.13 \pm 0.07$ & $1.92 \pm 0.06$ & $2.59 \pm 0.24$ & $97 \pm 6$ & $48 \pm 3$ \\
\hline $1057.9 \ldots \ldots \ldots \ldots \ldots \ldots \ldots$ & $0.05 \pm 0.01$ & $1206.4 \pm 1.8$ & $2.24 \pm 0.07$ & $2.02 \pm 0.06$ & $2.64 \pm 0.24$ & $95 \pm 6$ & $47 \pm 3$ \\
\hline $1027.8 \ldots \ldots \ldots \ldots \ldots \ldots \ldots$ & $0.04 \pm 0.01$ & $1205.3 \pm 1.8$ & $2.39 \pm 0.07$ & $2.15 \pm 0.07$ & $2.71 \pm 0.24$ & $91 \pm 6$ & $45 \pm 3$ \\
\hline $900.4 \ldots \ldots \ldots \ldots \ldots \ldots \ldots$ & $0.01 \pm 0.01$ & $1204.2 \pm 2.0$ & $2.79 \pm 0.17$ & $2.52 \pm 0.15$ & $2.79 \pm 0.25$ & $80 \pm 6$ & $39 \pm 3$ \\
\hline
\end{tabular}

a The error in the radius is the random error only and does not include (1) a component from the uncertainty in the zero level of the light curve or (2) the systematic error of $\pm 10 \mathrm{~km}$ that arises from the imprecisely known position of the KAO (Elliot et al. 1989; Millis et al. 1993).

$\mathrm{b}$ The stellar flux is an average over the atmospheric shell bounded by the row above and the row in which the flux is entered (Fig. 2).

TABLE 12

Pluto InVERSION: EMersion

\begin{tabular}{|c|c|c|c|c|c|c|c|}
\hline $\begin{array}{l}\text { Observer Center } \\
\text { Distance, } y \\
(\mathrm{~km})\end{array}$ & $\begin{array}{c}\text { Stellar Flux } \\
\text { (Normalized) }\end{array}$ & $\begin{array}{c}\text { Radius }^{\mathrm{a}} \\
(\mathrm{km})\end{array}$ & $\begin{array}{c}\text { Refractivity } \\
\left(10^{-9}\right)\end{array}$ & $\begin{array}{l}\text { Number Density } \\
\quad\left(10^{14} \mathrm{~cm}^{-3}\right)\end{array}$ & $\begin{array}{c}\text { Pressure } \\
(\mu \text { bar })\end{array}$ & $\begin{array}{c}\text { Temperature } \\
(\mathrm{K})\end{array}$ & $\begin{array}{l}\text { Scale Height } \\
(\mathrm{km})\end{array}$ \\
\hline $1191.3 .$. & $\mathrm{b}$ & $1245.0 \pm 1.7$ & $1.10 \pm 0.07$ & $0.99 \pm 0.06$ & $1.46 \pm 0.24$ & $107 \pm 11$ & $56 \pm 6$ \\
\hline $1188.7 \ldots$ & $0.51 \pm 0.04$ & $1243.8 \pm 1.7$ & $1.12 \pm 0.07$ & $1.01 \pm 0.06$ & $1.50 \pm 0.24$ & $107 \pm 11$ & $56 \pm 6$ \\
\hline $1186.2 \ldots$. & $0.52 \pm 0.04$ & $1242.5 \pm 1.7$ & $1.14 \pm 0.07$ & $1.03 \pm 0.06$ & $1.53 \pm 0.24$ & $108 \pm 11$ & $56 \pm 6$ \\
\hline 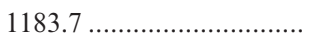 & $0.56 \pm 0.04$ & $1241.2 \pm 1.7$ & $1.17 \pm 0.07$ & $1.05 \pm 0.06$ & $1.57 \pm 0.24$ & $108 \pm 11$ & $56 \pm 6$ \\
\hline 1181.2 & $0.47 \pm 0.04$ & $1240.1 \pm 1.7$ & $1.19 \pm 0.07$ & $1.08 \pm 0.06$ & $1.60 \pm 0.25$ & $108 \pm 11$ & $56 \pm 6$ \\
\hline 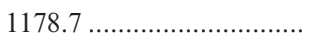 & $0.50 \pm 0.04$ & $1238.9 \pm 1.7$ & $1.22 \pm 0.07$ & $1.10 \pm 0.06$ & $1.63 \pm 0.25$ & $108 \pm 10$ & $56 \pm 6$ \\
\hline 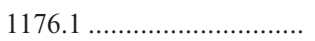 & $0.46 \pm 0.04$ & $1237.8 \pm 1.7$ & $1.24 \pm 0.07$ & $1.12 \pm 0.06$ & $1.66 \pm 0.25$ & $108 \pm 10$ & $56 \pm 6$ \\
\hline 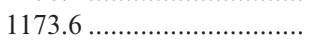 & $0.43 \pm 0.04$ & $1236.7 \pm 1.7$ & $1.27 \pm 0.07$ & $1.14 \pm 0.06$ & $1.70 \pm 0.25$ & $108 \pm 10$ & $56 \pm 5$ \\
\hline 1168.7 & $0.45 \pm 0.03$ & $1234.6 \pm 1.7$ & $1.32 \pm 0.07$ & $1.19 \pm 0.06$ & $1.76 \pm 0.25$ & $108 \pm 10$ & $56 \pm 5$ \\
\hline 1166.2 & $0.49 \pm 0.04$ & $1233.5 \pm 1.7$ & $1.34 \pm 0.07$ & $1.21 \pm 0.06$ & $1.80 \pm 0.25$ & $108 \pm 10$ & $55 \pm 5$ \\
\hline 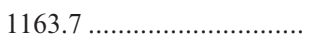 & $0.49 \pm 0.04$ & $1232.3 \pm 1.7$ & $1.37 \pm 0.07$ & $1.24 \pm 0.06$ & $1.84 \pm 0.26$ & $108 \pm 10$ & $55 \pm 5$ \\
\hline 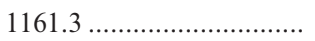 & $0.50 \pm 0.04$ & $1231.2 \pm 1.7$ & $1.40 \pm 0.07$ & $1.26 \pm 0.06$ & $1.87 \pm 0.26$ & $108 \pm 10$ & $55 \pm 5$ \\
\hline 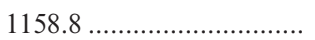 & $0.54 \pm 0.04$ & $1229.9 \pm 1.7$ & $1.43 \pm 0.07$ & $1.29 \pm 0.06$ & $1.92 \pm 0.26$ & $108 \pm 10$ & $55 \pm 5$ \\
\hline $1156.3 \ldots \ldots \ldots \ldots \ldots \ldots \ldots \ldots \ldots$ & $0.45 \pm 0.04$ & $1228.9 \pm 1.7$ & $1.46 \pm 0.07$ & $1.31 \pm 0.06$ & $1.95 \pm 0.26$ & $108 \pm 9$ & $55 \pm 5$ \\
\hline 1153.9 & $0.48 \pm 0.04$ & $1227.8 \pm 1.7$ & $1.49 \pm 0.07$ & $1.34 \pm 0.06$ & $1.99 \pm 0.26$ & $108 \pm 9$ & $55 \pm 5$ \\
\hline 1151.5 & $0.48 \pm 0.04$ & $1226.7 \pm 1.7$ & $1.51 \pm 0.07$ & $1.36 \pm 0.06$ & $2.03 \pm 0.26$ & $108 \pm 9$ & $55 \pm 5$ \\
\hline
\end{tabular}


TABLE 12-Continued

\begin{tabular}{|c|c|c|c|c|c|c|c|}
\hline $\begin{array}{l}\text { Observer Center } \\
\text { Distance, } y \\
(\mathrm{~km})\end{array}$ & $\begin{array}{c}\text { Stellar Flux } \\
\text { (Normalized) }\end{array}$ & $\begin{array}{c}\text { Radius }^{\mathrm{a}} \\
(\mathrm{km})\end{array}$ & $\begin{array}{c}\text { Refractivity } \\
\left(10^{-9}\right)\end{array}$ & $\begin{array}{l}\text { Number Density } \\
\quad\left(10^{14} \mathrm{~cm}^{-3}\right)\end{array}$ & $\begin{array}{c}\text { Pressure } \\
(\mu \text { bar })\end{array}$ & $\begin{array}{c}\text { Temperature } \\
(\mathrm{K})\end{array}$ & $\begin{array}{l}\text { Scale Height } \\
\quad(\mathrm{km})\end{array}$ \\
\hline 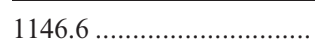 & $0.45 \pm 0.03$ & $1224.7 \pm 1.8$ & $1.57 \pm 0.07$ & $1.42 \pm 0.06$ & $2.11 \pm 0.27$ & $108 \pm 9$ & $55 \pm 5$ \\
\hline 1141.8 & $0.42 \pm 0.03$ & $1222.8 \pm 1.8$ & $1.62 \pm 0.07$ & $1.46 \pm 0.06$ & $2.18 \pm 0.27$ & $108 \pm 9$ & $55 \pm 5$ \\
\hline $1137.0 \ldots \ldots \ldots \ldots \ldots \ldots \ldots \ldots$ & $0.40 \pm 0.03$ & $1221.0 \pm 1.8$ & $1.68 \pm 0.07$ & $1.51 \pm 0.06$ & $2.26 \pm 0.27$ & $108 \pm 9$ & $55 \pm 5$ \\
\hline 1132.2 & $0.36 \pm 0.03$ & $1219.4 \pm 1.8$ & $1.73 \pm 0.07$ & $1.56 \pm 0.06$ & $2.32 \pm 0.28$ & $108 \pm 9$ & $54 \pm 4$ \\
\hline 1127.4 & $0.45 \pm 0.03$ & $1217.4 \pm 1.8$ & $1.79 \pm 0.07$ & $1.62 \pm 0.06$ & $2.41 \pm 0.28$ & $108 \pm 8$ & $54 \pm 4$ \\
\hline $1125.1 \ldots$ & $0.54 \pm 0.04$ & $1216.2 \pm 1.8$ & $1.83 \pm 0.07$ & $1.65 \pm 0.06$ & $2.46 \pm 0.28$ & $108 \pm 8$ & $54 \pm 4$ \\
\hline 1120.4. & $0.30 \pm 0.03$ & $1214.9 \pm 1.8$ & $1.87 \pm 0.07$ & $1.69 \pm 0.06$ & $2.52 \pm 0.28$ & $108 \pm 8$ & $54 \pm 4$ \\
\hline $1115.7 \ldots \ldots \ldots \ldots \ldots \ldots \ldots \ldots$ & $0.30 \pm 0.03$ & $1213.6 \pm 1.8$ & $1.92 \pm 0.07$ & $1.73 \pm 0.06$ & $2.58 \pm 0.28$ & $108 \pm 8$ & $54 \pm 4$ \\
\hline 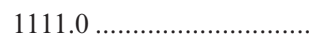 & $0.24 \pm 0.03$ & $1212.6 \pm 1.8$ & $1.96 \pm 0.07$ & $1.77 \pm 0.06$ & $2.63 \pm 0.29$ & $108 \pm 8$ & $54 \pm 4$ \\
\hline 1104.1 & $0.21 \pm 0.02$ & $1211.3 \pm 1.8$ & $2.03 \pm 0.07$ & $1.83 \pm 0.06$ & $2.70 \pm 0.29$ & $107 \pm 8$ & $53 \pm 4$ \\
\hline 1095.0 & $0.14 \pm 0.02$ & $1210.2 \pm 1.8$ & $2.09 \pm 0.07$ & $1.88 \pm 0.07$ & $2.76 \pm 0.29$ & $106 \pm 8$ & $53 \pm 4$ \\
\hline $1086.0 \ldots \ldots \ldots \ldots \ldots \ldots \ldots$ & $0.14 \pm 0.02$ & $1209.0 \pm 1.8$ & $2.16 \pm 0.07$ & $1.95 \pm 0.07$ & $2.82 \pm 0.29$ & $105 \pm 7$ & $52 \pm 4$ \\
\hline 1077.2. & $0.13 \pm 0.02$ & $1208.0 \pm 1.8$ & $2.23 \pm 0.07$ & $2.01 \pm 0.07$ & $2.88 \pm 0.29$ & $103 \pm 7$ & $51 \pm 4$ \\
\hline 1062.0 . & $0.09 \pm 0.02$ & $1206.8 \pm 1.9$ & $2.33 \pm 0.07$ & $2.10 \pm 0.07$ & $2.94 \pm 0.30$ & $101 \pm 7$ & $50 \pm 4$ \\
\hline 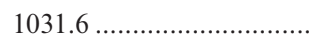 & $0.04 \pm 0.01$ & $1205.8 \pm 1.9$ & $2.47 \pm 0.08$ & $2.22 \pm 0.07$ & $3.00 \pm 0.30$ & $98 \pm 7$ & $48 \pm 4$ \\
\hline
\end{tabular}

a The error in the radius is the random error only and does not include (1) a component from the uncertainty in the zero level of the light curve or (2) the systematic error of $\pm 10 \mathrm{~km}$ that arises from the imprecisely known position of the KAO (Elliot et al. 1989; Millis et al. 1993).

$\mathrm{b}$ The stellar flux is an average over the atmospheric shell bounded by the row above and the row in which the flux is entered (Fig. 2).
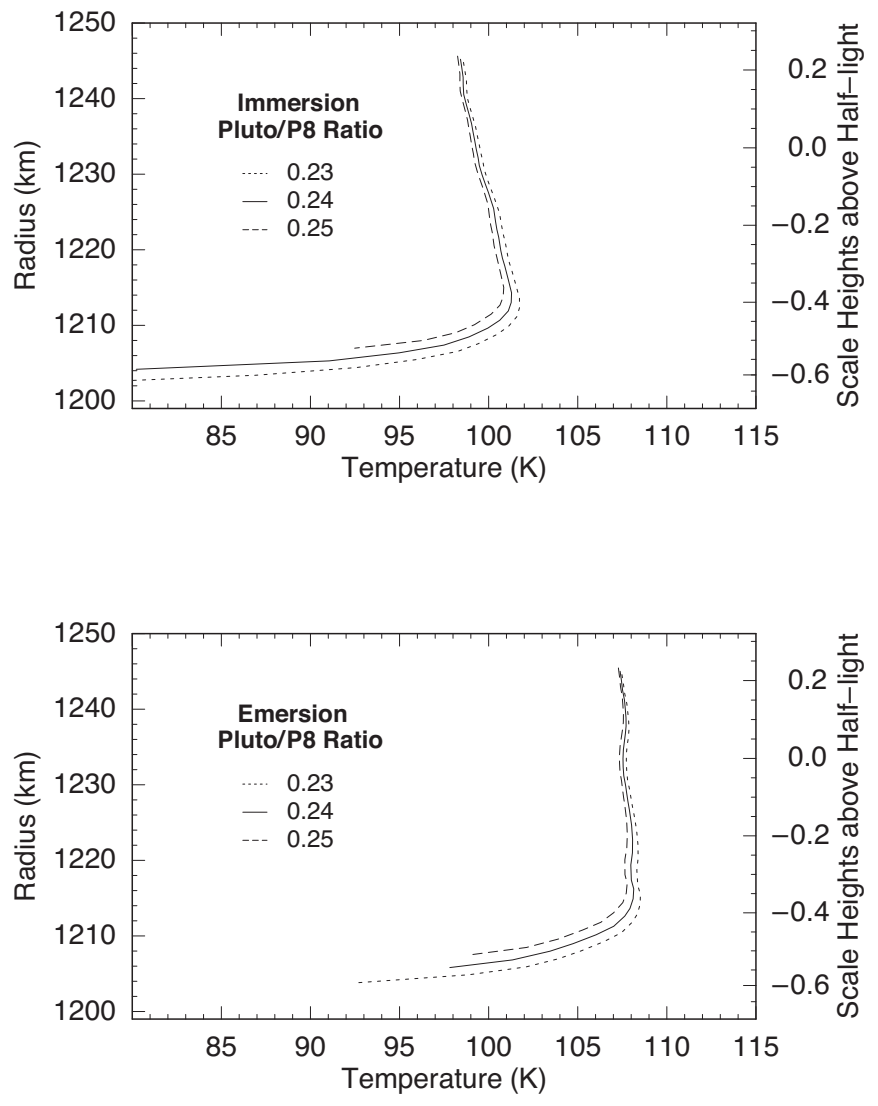

FIG. 13.- Systematic effects of the Pluto/P8 signal ratio. The top panel (immersion) and bottom panel (emersion) show the temperature of Pluto's atmosphere plotted vs. radius for three different values of the ratio of the light from Pluto-Charon and the occulted star, P8. The right scale shows scale heights above half-light equivalent of the radius scale on the left side. For clarity, only the lines joining the results of the inversions have been plotted. As the assumed signal ratio increases, the temperature profile moves to lower temperatures and larger radii, but the effect is small.
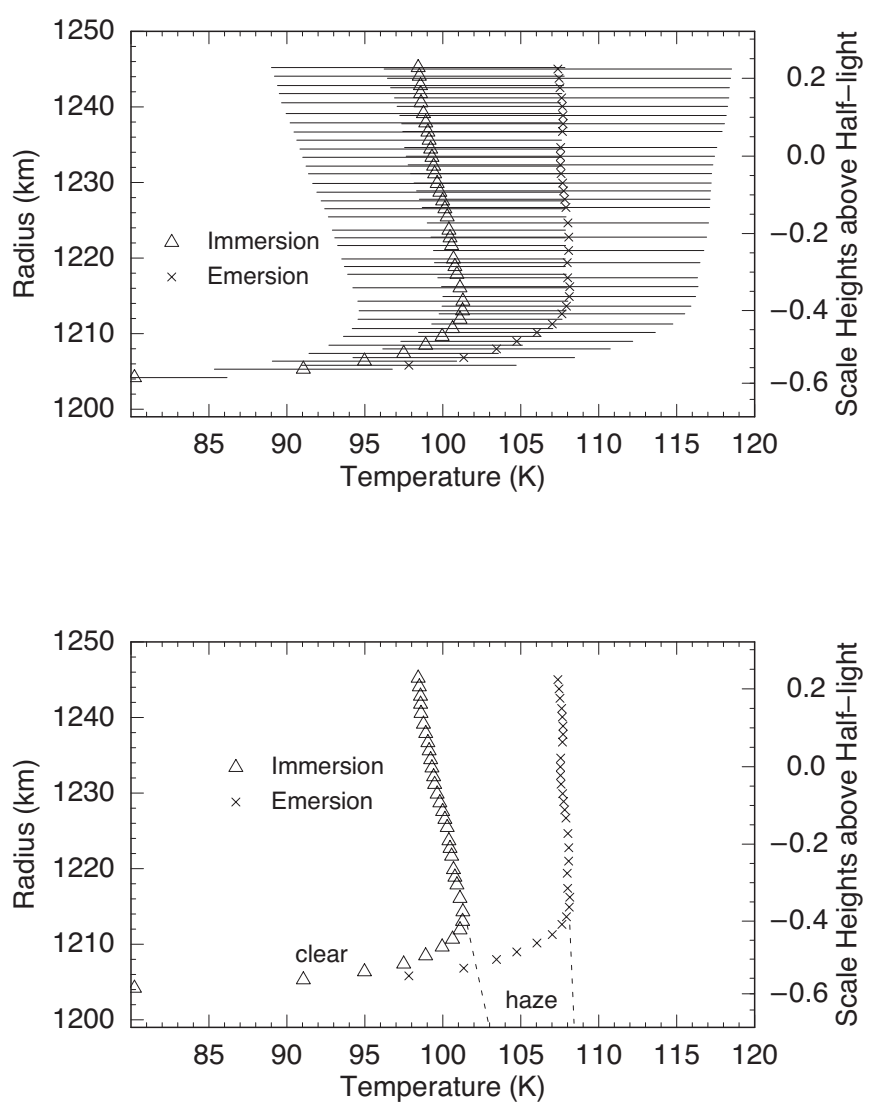

FIG. 14.-Pluto temperature profiles. Temperatures and their errors for inversions of the immersion and emersion are plotted vs. radius for a Pluto/P8 signal ratio of 0.24 . The right scale shows scale heights above half-light equivalent of the radius scale on the left side. Differences between the two profiles at all radii can be explained by noise in the occultation light curve. Error bars are shown in the top panel, but they are omitted for clarity in the bottom panel. The dashed line labeled "haze" in the bottom panel indicates the approximate temperature profile if the atmosphere is not clear (i.e., the sharp drop in the light curve is due to extinction, rather than the onset of a thermal gradient). Then the inversion temperature profile would be invalid, since it is based on the assumption of a clear atmosphere. 

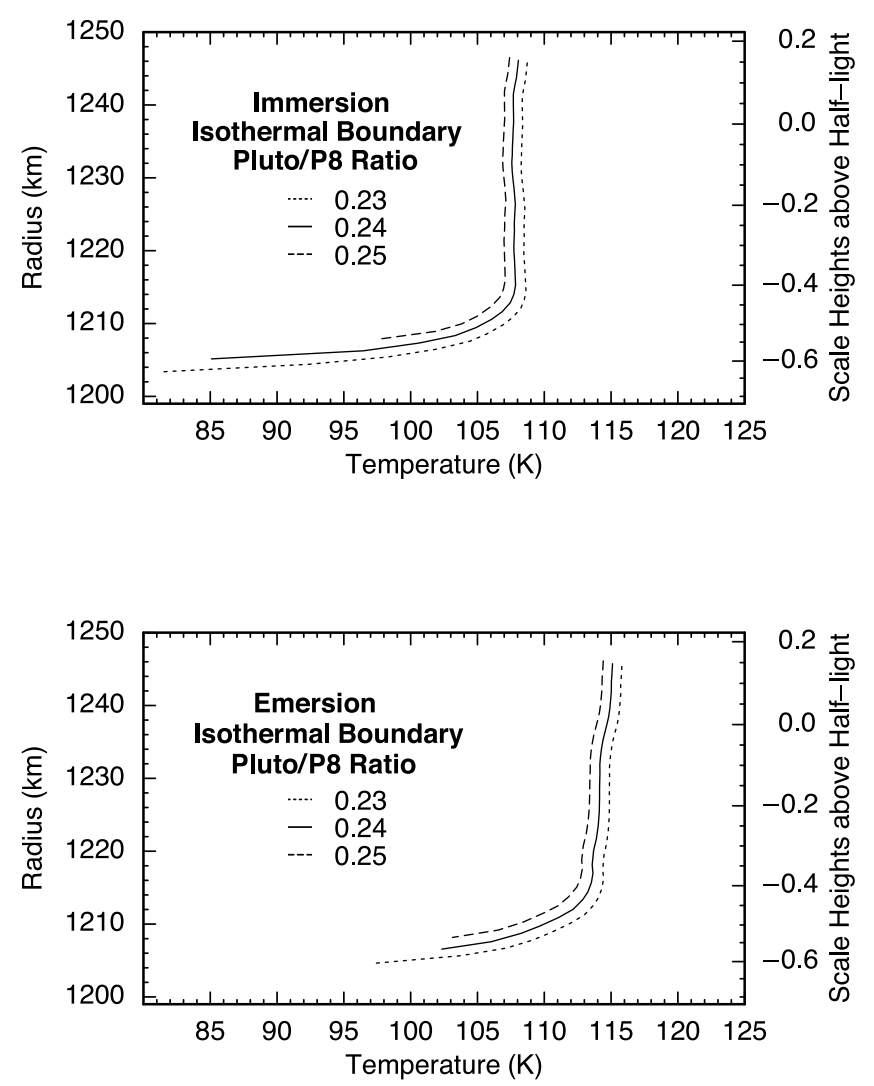

FIG. 15.-Same as Fig. 13, but for an isothermal boundary condition. As the signal ratio increases, the temperature profile moves to lower temperatures, but the effect is small.

atmosphere have not affected the light curve - which may not be true.

Finally we consider the accuracy of the radius scale (within Pluto's atmosphere) for the Pluto inversions. Although the formal errors in the radius scale are less than 2 $\mathrm{km}$ (Tables 11 and 12), we must remember that the closestapproach distance of the KAO to the center of Pluto's shadow $(865.69 \mathrm{~km})$ has an uncertainty of $\pm 15 \mathrm{~km}$-an error arising (primarily) from the uncertainty in the KAO flight path (Elliot et al. 1989; Paper I) and the uncertainty of the center of Pluto's shadow from the global solution that included the entire occultation data set (Millis et al. 1993). The closest-approach uncertainty in the observer plane $(y$ coordinate) translates into a $\pm 10 \mathrm{~km}$ uncertainty within Pluto's atmosphere ( $r$-coordinate).

\section{APPLICATION TO THE 1997 TRITON OCCULTATION}

Another high-quality data set amenable to inversion with the method described here is the 1997 November 4 occultation of the star Tr180 (McDonald \& Elliot 1995) by Triton, which was observed with the FGS on HST (Elliot et al. 1998, 2000b). These data are displayed in Figure 18, where the light curve has been normalized to zero and full-scale stellar flux by establishing the flux ratio of Triton and $\operatorname{Tr} 180$ in the FGS prior to the occultation. The time resolution of the light curve displayed is $1.0 \mathrm{~s}$, although the data were recorded at a time resolution of $0.025 \mathrm{~s}$ (Elliot et al. 1998).
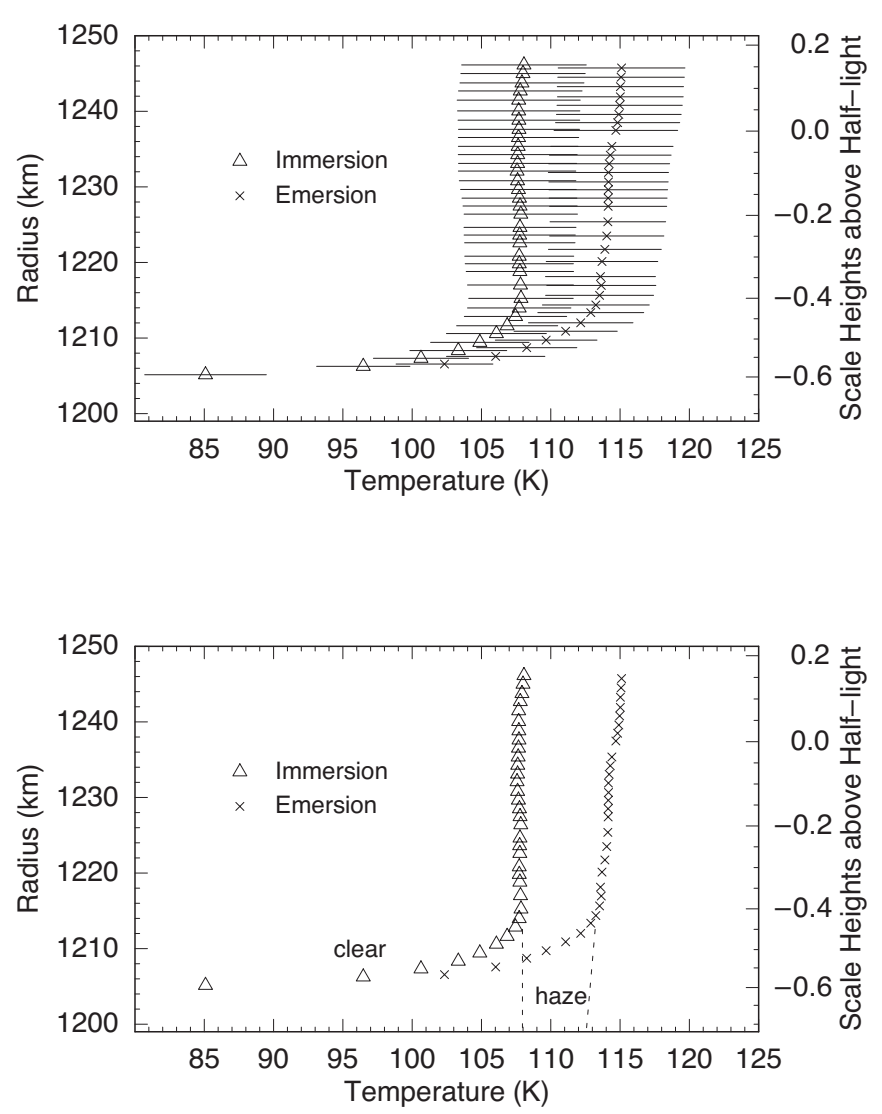

FIG. 16.-Same as Fig. 14, but for an isothermal boundary condition. Note that the error bars for the isothermal boundary are about half those for the case in which a thermal gradient is allowed in the boundary condition (Fig. 14).

The random noise is barely discernible in the unocculted portions of the light curve, and the deviations from a smooth curve during the occultation are caused by the density structure in Triton's atmosphere. The residuals of this light curve from an isothermal fit are displayed in Figure 1 of Elliot et al. (1998), and they show a marked deviation from an isothermal model. Hence we can gain information about Triton's atmosphere through inversion of the light curve (see $\S 7.2$ ).

Inversion of the light curve to establish Triton's atmospheric structure has been done previously by Elliot et al. (2000b), with an earlier version of the inversion method described here. Those results revealed a thermal gradient in the atmosphere above $50 \mathrm{~km}$ altitude (about $1402 \mathrm{~km}$ radius), where the atmospheric structure is apparently controlled by $\mathrm{CO}$ radiative cooling and the conduction of heat deposited by solar UV and magnetospheric electrons (Strobel \& Summers 1995). Below this altitude the profile is nearly isothermal, with only a slight increase in temperature with decreasing altitude. Here we shall further scrutinize the inversion results and compare the thermal profiles for different assumptions about the radius scale for the occultation.

As described in Elliot et al. (2000b), the nominal closest approach of HST to the center of Triton's shadow was 224 $\mathrm{km}$, with an uncertainty of $\pm 15 \mathrm{~km}$. In setting the radius scale, there is also the issue of the nonspherical figure of Triton's atmosphere (Elliot et al. 2000a) on the results of the inversion, especially at the lower altitudes. Six inversions of 

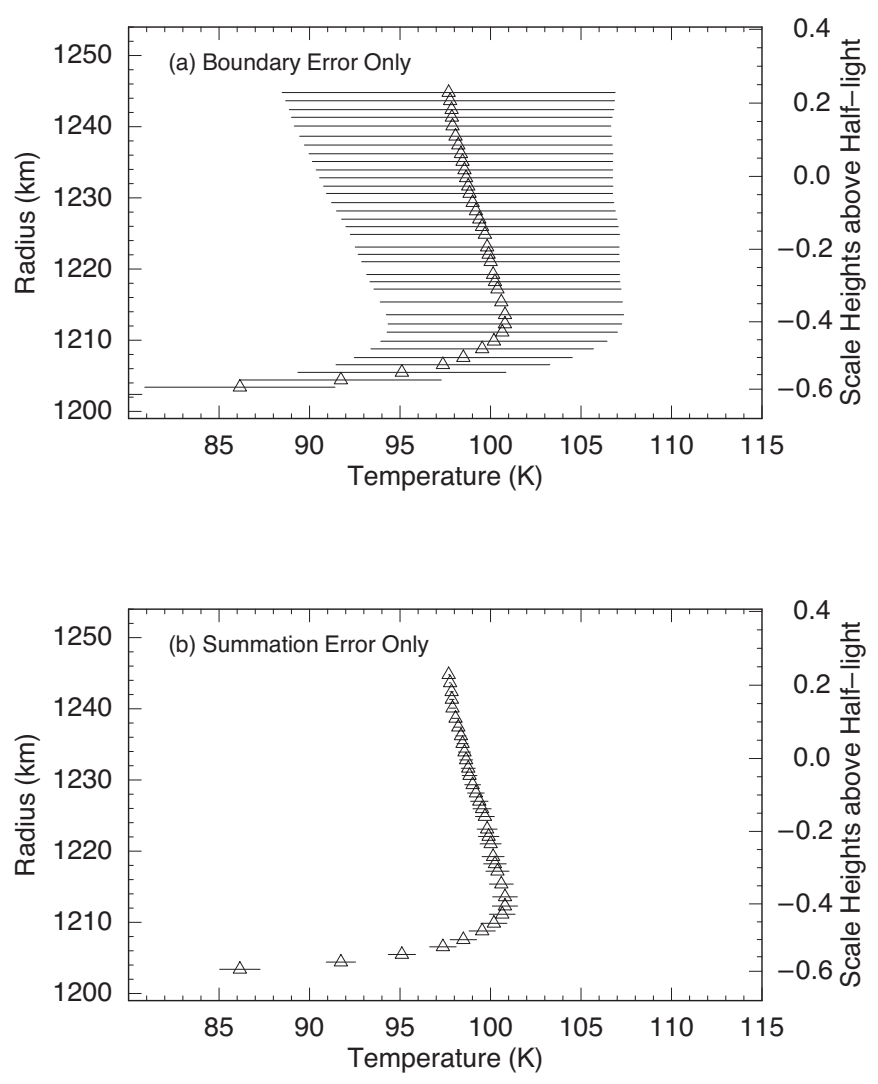

FIG. 17.-Errors for the boundary and summation terms for the inversion of Pluto's light curve. The inversion of the Pluto light curve for immersion with the Pluto/P8 signal ratio of 0.24 is plotted with the error bar contributions from the boundary (only) in the top panel, and the error bar contributions from the summation terms (only) in the bottom panel. The right scale shows scale heights above half-light equivalent of the radius scale on the left side. For this case, the errors are dominated by the contribution from the boundary term.

the occultation light curve were carried out: three for immersion and three for emersion. Each set of three used the closest-approach distances of 209, 224, and 239 $\mathrm{km}$. The light curve was kept at $1.0 \mathrm{~s}$ resolution, and the

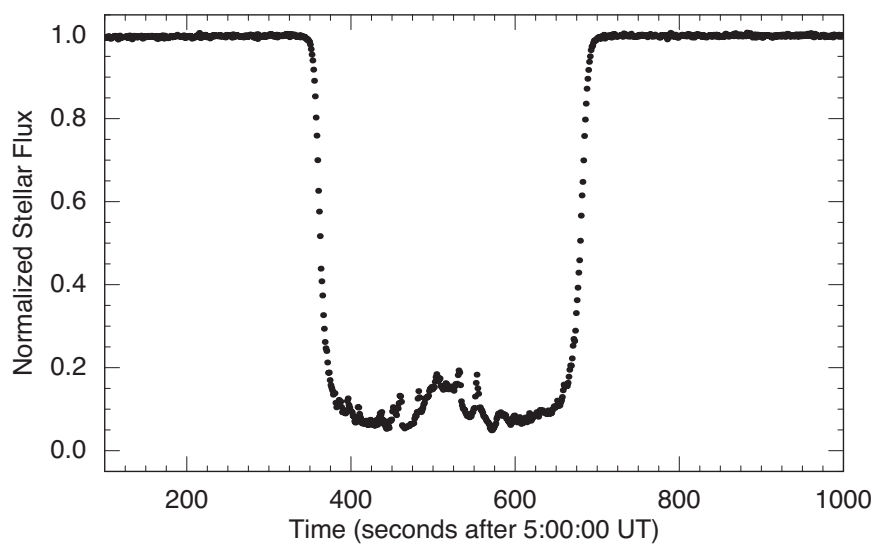

FIG. 18.-Triton occultation. This plot shows $1 \mathrm{~s}$ averages of the HST light curve for Triton's occultation of the star Tr180 (McDonald \& Elliot 1995), which occurred on 1997 November 4 and was observed with the FGS. The small "scruff" before and after the occultation arises from photon noise, while the large deviations from a smooth curve at the lowest flux levels are due to nonisothermal structure within Triton's atmosphere (adapted from Elliot et al. 2000b). observer-plane coordinates were calculated for the closestapproach distance used for each inversion. A far-limb flux corresponding to the (adopted) model fit published previously (Elliot et al. 1998) was subtracted from the immersion and emersion sections of the light curve. Inversions were carried out with the parameters given in Tables 9 and 10 . The results of these six inversions are displayed in Figure 19, with the immersion profiles plotted in the top panel and the emersion profiles plotted in the bottom panel. The solid line in both panels indicates the nominal closest-approach distance $(224 \mathrm{~km})$. For increasing distances from the shadow center, the profiles are displaced to higher temperatures and lower radii. These inversion results are also tabulated in Table 13 (immersion) and Table 14 (emersion).

In Figure 20, we have plotted the temperature profiles for the nominal closest-approach distance, along with the error bars on the temperatures. The immersion and emersion profiles differ at the largest radii and at the smallest radii. However, the differences at the largest radii are within what would be expected from the errors (see Fig. 20), while the differences at the smallest radii are significantly larger than
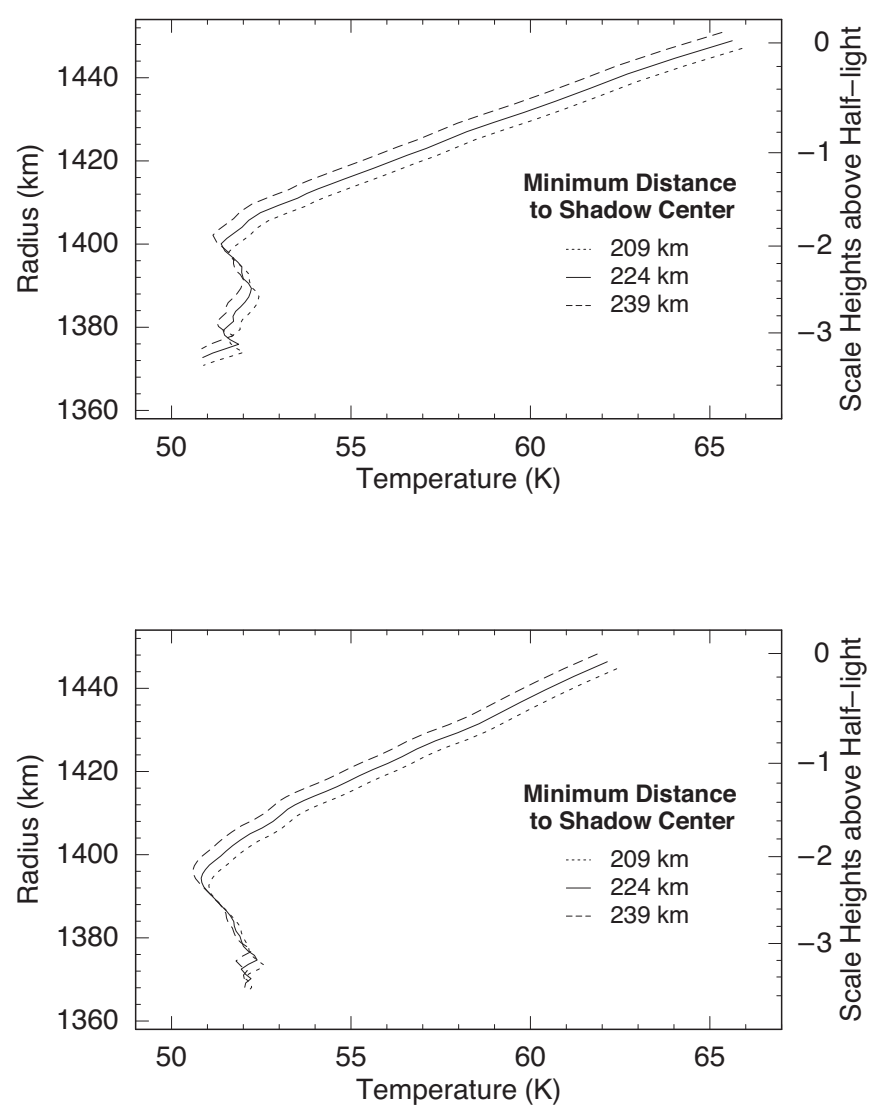

FIG. 19.- -Systematic effects of closest-approach distance for Triton. The top panel (immersion) and bottom panel (emersion) show the temperature of Triton's atmosphere plotted vs. radius for three different values of the closest approach of HST to the center of Triton's shadow. The right scale shows scale heights above half-light that correspond to the radius scale on the left. For clarity, only the lines joining the results of the inversions have been plotted. As the closest-approach distance increases, each point on the temperature profile is displaced to a larger radius and lower temperaturepreserving the character of the gradients and more complex thermal variability. The standard case for a closest-approach distance of $224 \mathrm{~km}$ is indicated by the solid line in both panels, and the cases for 1 standard deviation high and low are indicated by the dashed lines (Elliot et al. 2000b). 
TABLE 13

Triton Inversion: Immersion, 224 km Closest Approach

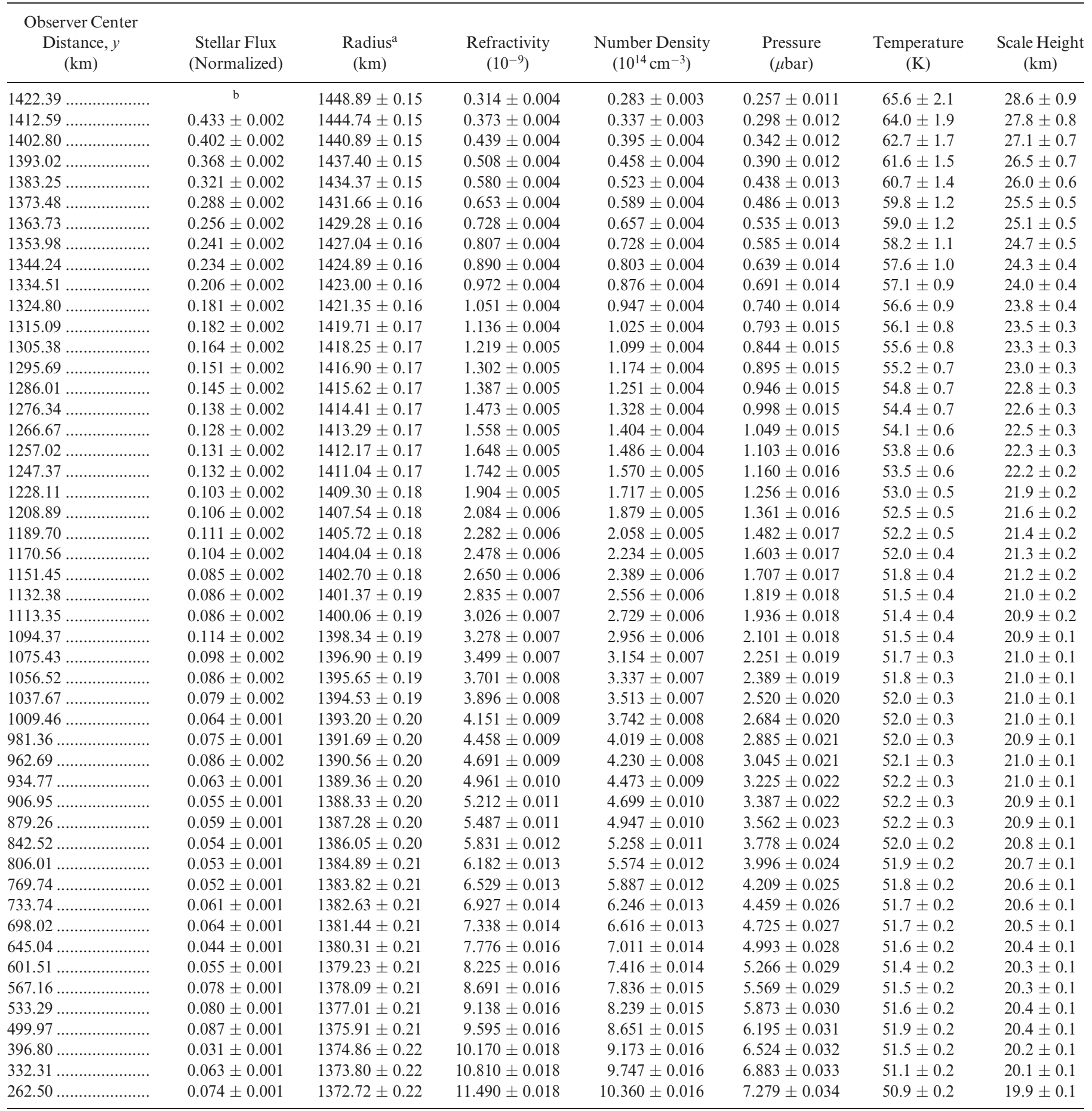

a The error in the radius is the random error only and does not include a component from the uncertainty in the zero level of the light curve.

b The stellar flux is an average over the atmospheric shell bounded by the row above and the row in which the flux is entered (Fig. 2).

what would be expected from the formal errors. The differences at lower altitudes are compared in more detail in Figure 21, where the top panel shows the profiles with the error bars and the bottom panel shows the profiles without the error bars, for greater clarity. We see a wavelike structure at the lowest altitudes, with peak-to-peak amplitude of $\sim 1 \mathrm{~K}$ and a vertical wavelength of $\sim 20 \mathrm{~km}$. We note that these structures may reflect vertical and/or horizontal structure in the atmosphere, since a large horizontal segment of the limb is being sampled near the center of the occultation light curve (which corresponds to the lowest altitudes in both profiles).

\section{ATMOSPHERES OF PLUTO AND TRITON}

Pluto and Triton are quite similar in size, density, and (in recent years) solar distance. Each has $\mathrm{N}_{2}$ as its major atmospheric constituent, with $\mathrm{CH}_{4}$ and $\mathrm{CO}$ as minor constituents 
TABLE 14

Triton InVersion: Emersion, 224 km Closest ApProach

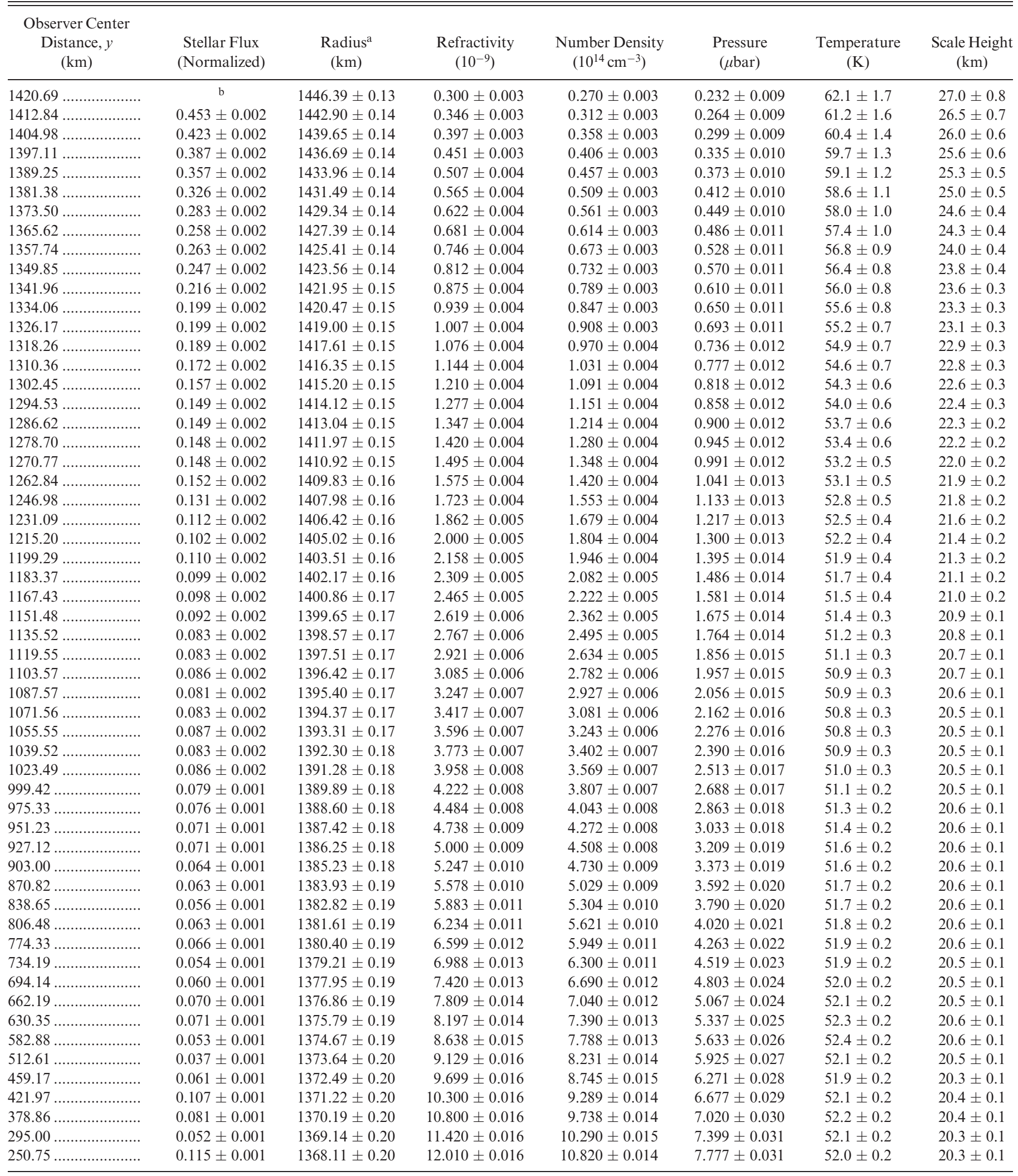

a The error in the radius is the random error only and does not include a component from the uncertainty in the zero level of the light curve.

$\mathrm{b}$ The stellar flux is an average over the atmospheric shell bounded by the row above and the row in which the flux is entered (Fig. 2). 


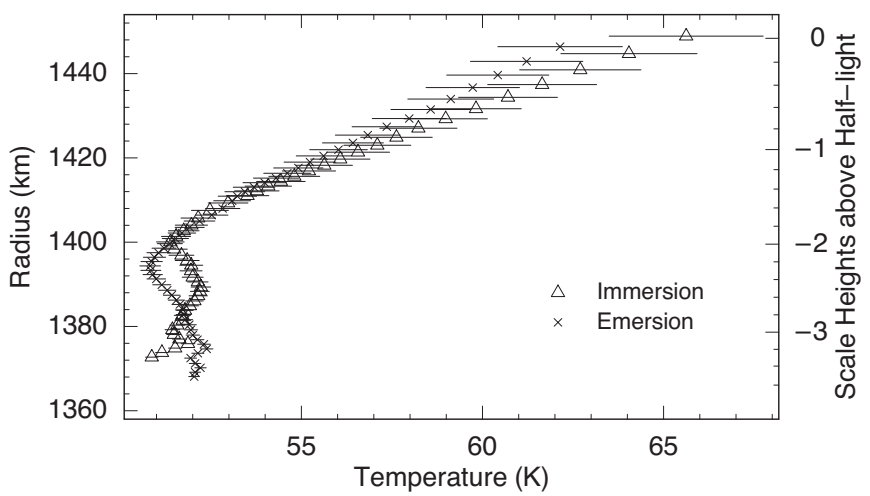

FIG. 20.-Triton temperature profiles. All temperatures and their errors for inversions of the immersion and emersion portions of the light curve shown in Fig. 18 are plotted vs. radius for the standard closest-approach distance of $224 \mathrm{~km}$. The right scale shows scale heights above half-light equivalent of the radius scale on the left side. Differences between the two profiles at large radii can be explained by noise in the occultation light curve, while variations at smaller radii exceed the noise-induced variations and indicate differences in thermal structure between the two regions in Triton's atmosphere probed by the occultation.

(Cruikshank et al. 1993; Owen et al. 1993) that are radiatively active (Strobel et al. 1996; Strobel \& Summers 1995). However, their atmospheric structures differ considerably. A fundamental observational comparison of their light
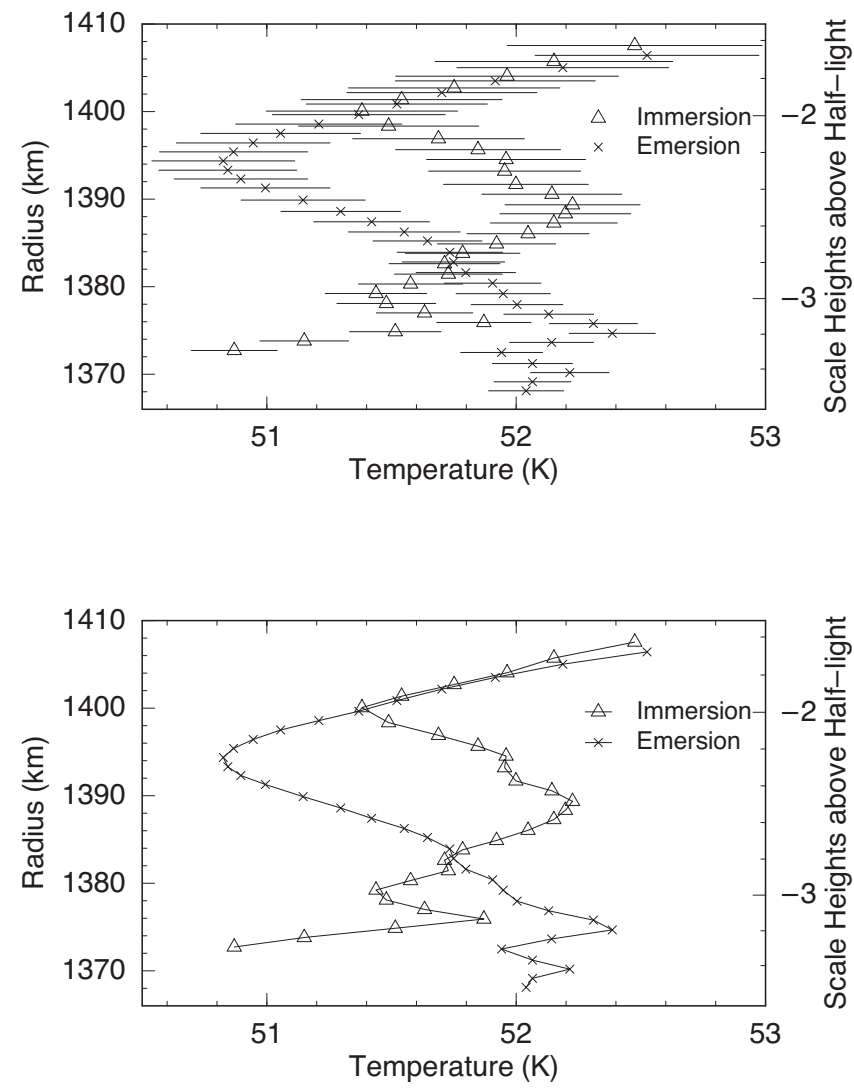

FIG. 21.-Comparison of Triton thermal variability. All temperatures for inversions of the immersion and emersion portions of the light curve shown in Fig. 18 are plotted vs. radius for the standard closest-approach distance of $224 \mathrm{~km}$ for radii below $1410 \mathrm{~km}$. The right scale shows scale heights above half-light equivalent of the radius scale on the left side. The top panel shows the error bars, while the bottom panel omits the error bars and connects the points to bring out the character of the thermal variations. For reference, Triton's surface radius is $1352 \mathrm{~km}$.

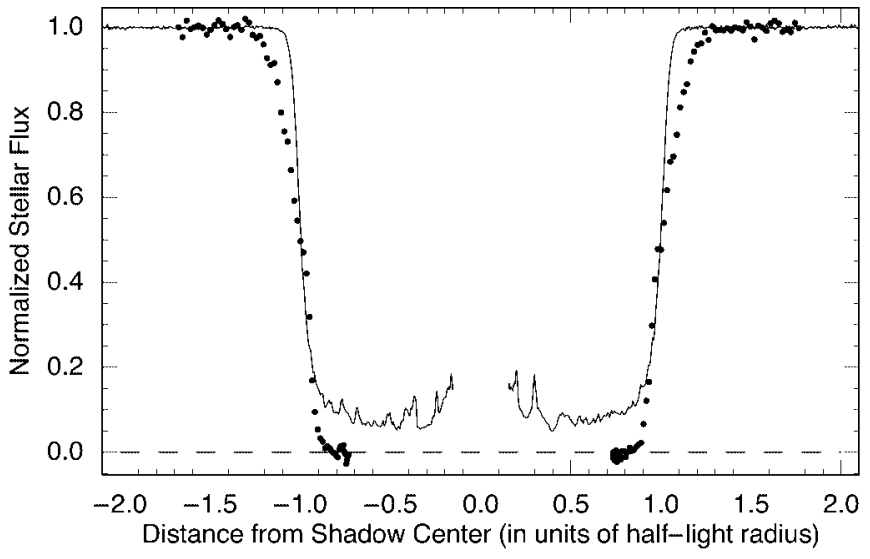

FIG. 22.-Pluto and Triton occultation light curves. The occultation light curves for Pluto and Triton (Figs. 12 and 18) are plotted vs. distance from the center of their respective occultation shadows. The distance scale has been normalized in units of half-light radius (in the occultation shadow, which is a scale height smaller than the half-light radius in the body's atmosphere, because of refraction). The immersion and emersion data have both been plotted; minus signs have been added before the distances of the former to distinguish them from the latter. The Pluto data appear as points, while the Triton data appear as a line. The steeper slope near the top part of Triton's light curve indicates that its atmosphere has a smaller scale height than that of Pluto in the regions probed by their respective occultation light curves. Just below half-light, the Pluto light curve sharply drops to zero (see text). Note that the Triton occultation probed much deeper into the body's shadow than did the Pluto occultation.

curves reveals large differences, as illustrated in Figure 22, where we have plotted the Pluto KAO occultation light curve and Triton HST occultation light curve on a common scale. The Pluto data are plotted as averaged points, and the Triton data are plotted as a solid line. The ordinate is stellar flux, normalized between 0.0 (fully occulted) and 1.0 (unocculted), and the abscissa is the distance from the center of the occultation shadow (proceeding from immersion to emersion) normalized in units of half-light radius in the observer plane. Plotted on these normalized scales the curves cross at both half-light points $( \pm 1.0,0.5)$, as they must.

We note four things about Figure 22. First, the Triton data are much less noisy than the Pluto data, which is due to the fact that many more stellar photons were detected per normalized distance of shadow motion for Triton than for Pluto. Second, the light curve for Pluto probed barely $25 \%$ of the way between the half-light radius and the center of the shadow, while the Triton light curve probed much more deeply. Third, the slope for the Triton light curve between half-light and the unocculted level is steeper than that for Pluto, which a result of the much larger energy ratio $\left(\lambda_{\text {hi }}\right.$; see Table 8$)$ for Triton $(\sim 66)$ than for Pluto $(\sim 20)$. This indicates that Triton's atmosphere is more than 3 times more tightly bound than Pluto's in the regions probed by these stellar occultations. Finally, below half-light the light curve for Pluto abruptly drops to 0.0 , while that for Triton stays well above 0.0 -indicating fundamental differences in their atmospheric structure for this atmospheric region. These differences in the light curves imply differences in the temperature profiles from the inversions that can be seen in Figures 14 and 20. The caveat here (as always in this work) is that we are assuming that any extinction 
effects in these atmospheres are negligible, which may not be true for Pluto, as will be discussed in more detail in the next subsection.

\subsection{Pluto's Atmosphere}

Our current knowledge of Pluto's atmospheric structure at altitudes probed by the stellar occultation is summarized in Figure 23, where we have indicated three scenarios for temperature profiles that would be consistent with the KAO occultation data: (1) "no troposphere," (2) "deep troposphere," and (3) "haze." Temperature is plotted versus radius, with a pressure scale on the right that applies to the "deep troposphere" scenario. The temperature profiles from our inversions are indicated by triangles (immersion) and crosses (emersion). The error bars have not been plotted (see Figs. 14 and 16, top), but the difference between the two sets of points indicates their approximate uncertainty. Note that the main error is in the placement of the profiles, not their shape. The sections of the temperature profiles that correspond to the three scenarios have been drawn as solid lines. An $\mathrm{N}_{2}$ ice temperature of $40 \pm 2 \mathrm{~K}$ (Tryka et al. 1994) at the radius for vapor pressure equilibrium is indicated by the square (the error bar in radius corresponds to the error bar in temperature for vapor pressure equilibrium). The filled triangle is plotted at the surface radius of the mutual events (Young \& Binzel 1994) at a temperature that corresponds to that for $\mathrm{N}_{2}$ ice in vapor pressure equilibrium (the error bar in temperature corresponds to the error bar in radius for vapor pressure equilibrium).

Since the critical discussion of these scenarios by Young (1994), Strobel et al. (1996) have constructed a comprehensive radiative-conductive model for Pluto's atmosphere. They consider the downward conduction of solar UV absorbed in the upper atmosphere, radiative heating by the 2.3 and $3.3 \mu \mathrm{m}$ bands of $\mathrm{CH}_{4}$, radiative cooling by the $7.6 \mu \mathrm{m}$ band of $\mathrm{CH}_{4}$, and radiative cooling by $\mathrm{CO}$ rotational lines. Their model also imposes the constraint of the column abundance of $\mathrm{CH}_{4}$ measured by Young et al. (1997). Their results are included in the following discussion.

For the "no troposphere" model, the atmosphere is assumed to be clear (at least to the bottom of the region probed by the stellar occultation), so that the inversion results derived here would be valid. The temperature would be approximately isothermal above the $2.5 \mu$ bar level. Below, the temperature would suddenly drop, with an increasing thermal gradient that would rise to at least $3.9 \pm 0.6 \mathrm{~K} \mathrm{~km}^{-1}$ at $\sim 93 \mathrm{~K}(r=1206 \pm 10 \mathrm{~km})$, as indicated by the average of the final gradients in our immersion and emersion inversion profiles. The steep gradient would continue until it reached the surface temperature of $\sim 35 \mathrm{~K}$ for $\mathrm{N}_{2}$ ice, and $\sim 50-55 \mathrm{~K}$ for the bare areas. For this scenario the surface radius would be about $1195 \pm 10 \mathrm{~km}$ (Table 15). For pure $\mathrm{N}_{2}$ gas, a thermal gradient of $3.9 \mathrm{~K}$ $\mathrm{km}^{-1}$ at $93 \mathrm{~K}$ implies a heat flow of $0.035 \mathrm{ergs} \mathrm{cm}^{-2} \mathrm{~s}^{-1}$ for the thermal conductivity relation $\left(\kappa=5.63 T^{1.12} \mathrm{ergs} \mathrm{cm}^{-1}\right.$ $\mathrm{s}^{-1} \mathrm{~K}^{-2.12}$ ) used by Hubbard et al. (1990b). If we require the same heat flow at a surface temperature of $35 \mathrm{~K}$ (i.e., no net heat exchange within the intervening atmosphere from radiative processes), the thermal gradient at the surface would be $11.7 \mathrm{~K} \mathrm{~km}^{-1}$. For surface areas at $50 \mathrm{~K}$, the gradient would be $7.8 \mathrm{~K} \mathrm{~km}^{-1}$. These values are well below the $30 \mathrm{~K}$ $\mathrm{km}^{-1}$ used by Stansberry et al. (1994) in their modeling of Pluto's atmosphere and at the low end of the range of gradients considered by Strobel et al. (1996).

For this scenario, the model favored by Strobel et al. (1996) has a $3 \mu$ bar surface pressure and $3 \% \mathrm{CH}_{4}$ mixing ratio, a value consistent with the column abundance for

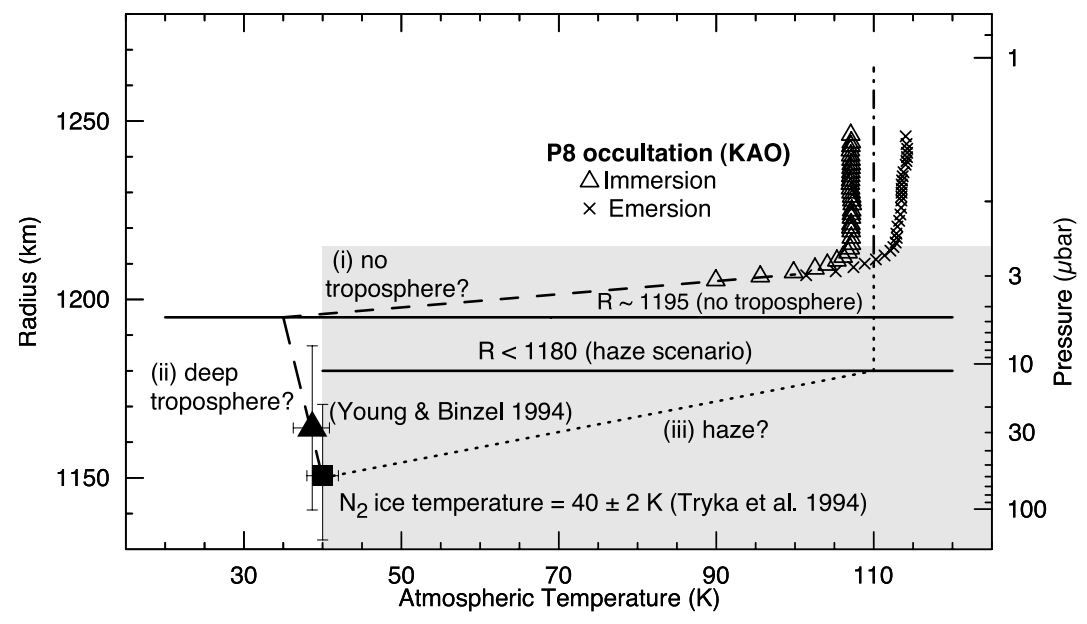

FIG. 23.-Possible thermal profiles for Pluto's atmosphere in 1988. Temperature (abscissa) is plotted vs. radius (ordinate) for three different thermal scenarios: (1) "no troposphere," (2) "deep troposphere," and (3) "haze." For the first two scenarios, extinction effects in the occultation light curve are assumed to be negligible. The pressure scale on the right is based on the deep-troposphere scenario. For all scenarios a predominantly $\mathrm{N}_{2}$ atmosphere in vapor pressure equilibrium with surface ice is assumed. The thermal inversion results (Fig. 14) for immersion and emersion are plotted as points (without error bars), while unmeasured parts of the profiles are plotted as lines. For the no-troposphere scenario, the thermal profile would follow the steep slope indicated by the inversion until the surface is reached ( $r=1150 \mathrm{~km}, p=60 \mu \mathrm{bar}$ ). On the other hand, if the atmosphere had a deep troposphere (Stansberry et al. 1994), the thermal profile would reach a minimum at the radius indicated by the no-troposphere scenario, but then it would continue a convective profile to the surface. The maximum temperature gradient would be the dry adiabatic gradient of $-g(r) / c_{p}=-0.61 \mathrm{~K} \mathrm{~km}^{-1}$ (for $r=1175 \mathrm{~km}$, where $c_{p}$ is the specific heat at constant pressure for $\mathrm{N}_{2}$ ), but smaller convective gradients have been discussed (Stansberry et al. 1994). In this scenario, the surface radius and pressure are not constrained and could match any value indicated by the mutual events (Young \& Binzel 1994). In the haze scenario, the steep drop in the occultation light curve is postulated to be caused by extinction effects in the atmosphere, so the temperature profile below the top of the haze layer $(r=1225 \mathrm{~km}, p=2.5 \mu \mathrm{bar})$ is unconstrained, although the temperature must decrease in some manner to reach a value at the surface that would be consistent with vapor pressure equilibrium for $\mathrm{N}_{2}$ ice at a surface temperature of $40 \pm 2 \mathrm{~K}$ (Tryka et al. 1994). 
TABLE 15

Comparison of Three Scenarios for Pluto's ATMOSPHERE

\begin{tabular}{|c|c|c|c|}
\hline Scenario $^{\mathrm{a}}$ & $\begin{array}{c}\text { Surface } \\
\text { Radius } \\
(\mathrm{km})\end{array}$ & $\begin{array}{c}\text { Surface } \\
\text { Pressure } \\
(\mu \mathrm{bar})\end{array}$ & $\begin{array}{l}\text { Density } \\
\left(\mathrm{g} \mathrm{cm}^{-3}\right)\end{array}$ \\
\hline No troposphere..................... & 1195 & 4.7 & 1.83 \\
\hline Deep troposphere.................. & $<1195$ & $>4.7$ & $>1.83$ \\
\hline 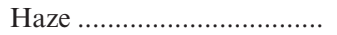 & $<1180$ & $>11.2$ & $>1.90$ \\
\hline
\end{tabular}

Note.-For Pluto's mass of $(1.312 \pm 0.003) \times 10^{25} \mathrm{~g}$ (see text). a See Fig. 23.

$\mathrm{CH}_{4}$ of $3.22_{-2.34}^{+8.46} \times 10^{19}$ molecules $\mathrm{cm}^{-2}$ measured by Young et al. (1997). However, Strobel et al.'s (1996) isothermal temperature $(\sim 134 \mathrm{~K})$ would be too high in the low-pressure region (a difficulty, as they discuss, that can be circumvented if the principal component of the atmosphere is $\mathrm{Ar}$ rather than $\mathrm{N}_{2}$ ). Another problem for the "no troposphere" scenario is that it requires surface radii beyond the high end of the range allowed by the mutual-event radius (Young 1994) and the measured $\mathrm{N}_{2}$ ice temperature (Tryka et al. 1994). We note here that the radius scale in Figure 23 has an error bar of $\pm 10 \mathrm{~km}$, which could bridge some of this gap.

The "deep troposphere" scenario (Fig. 23) was proposed by Stansberry et al. (1994) to avoid the disparity between the surface radius implied by the "no troposphere" model and the radii determined by the mutual events by and that implied by the $\mathrm{N}_{2}$ ice temperature. They postulated that the structure of the atmosphere would follow the "no troposphere " structure down to a radius of $\sim 1195 \mathrm{~km}$, where the top of a (possibly) deep troposphere would be reached. This boundary would cause a large positive excursion of the stellar occultation light curve, but the level would be below that probed by the KAO light curve. Hence, this view would be just as consistent with the KAO occultation data as the "no troposphere" scenario, and the other occultation data for the 1988 event either did not probe deeply enough or are not of sufficient quality (Millis et al. 1993) to confirm or rule out this possibility. A troposphere could be considered as a lower boundary condition for any radiative-conductive model, so the comments in that discussion would apply to this scenario as well.

Finally, we discuss the "haze" scenario (Fig. 23), which was proposed by Elliot et al. (1989) in their original analysis of the KAO data and modeled more precisely in Paper I. The normal optical depth required by the haze model would be greater than 0.145 , so the surface radius could be anything below the last detectable flux in the light curve, which occurs at $1181 \pm 10 \mathrm{~km}$ (Paper I). Stansberry et al. (1989) investigated the amount of particulates that would be required to match the light curve and found that it would be too great to be supported by such a tenuous atmosphere. To our knowledge, no one has yet considered whether strong winds (as have been inferred for Triton from the nonsphericity of its atmospheric figure by Elliot et al. 1997) could supply sufficient levitation for the amount of particles required by the "haze" scenario. Another difficulty for the "haze" scenario is the disagreement of the radius for optical depth 1.0 with that of the mutual events (Fig. 23). Since the undetectably low flux is so naturally achieved by extinction, it may be that the main drop in the light curve is caused by a steep thermal gradient, as in the two clear-atmosphere scenarios, but extinction by thin cloud or a modest haze layer, followed by total extinction of the stellar flux by the limb, takes the light-curve flux to zero (Hubbard et al. 1990b; Strobel et al. 1996).

In summary, the KAO data provide three constraints on physical models for Pluto's atmosphere, the first two derived from the light-curve modeling (Paper I) and the third derived here: (1) a temperature of $105 \pm 8 \mathrm{~K}$ at a radius of $1250 \pm 10 \mathrm{~km}$ ( $p=1.3 \mu \mathrm{bar})$, (2) a value for the thermal gradient in the 1.0-2.5 $\mu$ bar region of $-0.5 \pm 0.7 \mathrm{~K}$ $\mathrm{km}^{-1}$, and (3) a value for the thermal gradient of $3.9 \pm 0.6$ $\mathrm{K} \mathrm{km}^{-1}$ at $\sim 93 \mathrm{~K}(r=1206 \pm 10 \mathrm{~km})$. These values apply to a pure $\mathrm{N}_{2}$ atmosphere, but they can be adjusted for an atmosphere with a different mean molecular weight as described here and in Paper I. The error bar in the temperature has been reduced below the value quoted in Paper I because more accurate values for Pluto's mass have become available (Null \& Owen 1996; Foust et al. 1997; Olkin, Wasserman, \& Franz 2003). The unweighted mean for the three Pluto masses from these works is $(1.312 \pm 0.003) \times 10^{25} \mathrm{~g}$. With the reduced uncertainty in Pluto's mass, the temperature error is now dominated by the error in the pressure scale height given in Table 9 of Paper I. Apart from these three constraints, a more stringent test of any radiative-conductive model for Pluto's atmosphere would be a leastsquares fit of the model parameters to the KAO light curve by the method described by Chamberlain \& Elliot (1997).

\subsection{Triton's Atmosphere}

Observations of Triton's atmosphere by the Voyager 2 spacecraft (Smith et al. 1989; Tyler et al. 1989; Broadfoot et al. 1989) and the subsequent models (Strobel et al. 1990; Strobel \& Summers 1995; Yelle, Lunine, \& Hunten 1991; Yelle et al. 1995) produced a good first-order picture of Triton's atmosphere and the physical processes that determine its structure. This work was followed by a series of observations of stellar occultations by Triton (Olkin et al. 1997; Elliot et al. 2000a) that established the thermal structure in Triton's middle atmosphere (a region not probed by Voyager) and elucidated three issues that require further investigation: (1) a nonspherical shape of the half-light level (probably due to high winds; Elliot et al. 1997), (2) an increase in surface pressure since Voyager (likely caused by albedo changes; Elliot et al. 1998), and (3) the presence of an unknown coolant at an altitude of 50-60 km (Elliot et al. 2000b).

Here we add yet another atmospheric phenomenon: the temperature undulations in the lower atmosphere (see Fig. 21 , bottom). These undulations could be due to unremoved far-limb flux and/or horizontal (rather than vertical) structure, since the stellar image samples a large section of Triton's limb at the lowest altitudes. Interpretation of the atmospheric structure implied by these undulations must account for the integration of the differential refraction along the line of sight within Triton's atmosphere, according to equations (9) and (13). For a radius $r$ where the atmospheric scale height is $H(r)$, the region along the line of sight with a large contribution to the integral has an extent along the line of sight of about $[2 \pi r H(r)]^{1 / 2}$, which is $\sim 400 \mathrm{~km}$ for the region of Triton's atmosphere where the undulations occur. If the wave structure has a horizontal extent less than this, then it could have a larger amplitude than indicated in 
the temperature profiles. A thorough investigation of these temperature undulations is needed.

\section{CONCLUSIONS}

We have extended the inversion method for stellar occultation light curves for application to small-body atmospheres and have applied the technique to high-quality occultation light curves for Pluto and Triton. Our method uses a modular boundary condition to describe the atmosphere above the inversion region; hence, the boundary parameters can be derived from the data, a theoretical model, or a combination of both. Our prescription for calculating error bars on the resulting refractivity, number density, pressure, temperature, and scale height profiles allows us to know separately the amount of final error that is introduced from the boundary condition and from the stellar fluxes in the inversion region. Our inversion equations and code have been validated by the inversion of noiseless test cases, which produce temperatures with a numerical accuracy better than 1 part in $10^{4}$ from half-light to 4 scale heights below that level, and our error calculations have been validated by comparison with the scatter of results for inversion of a random sample of light curves.

As noted in previous investigations of stellar occultation light-curve inversion, the resulting atmospheric profiles are extremely sensitive to the boundary condition and any systematic errors in the zero level for the stellar flux. We found that setting the boundary radius at a value corresponding to half-light produces a good compromise between keeping the errors in the profiles arising from the boundary condition as small as possible and retaining a good portion of the light curve for inversion. The errors in the atmospheric profiles derived from the inversion scale linearly with the errors in the stellar fluxes. An unexpected result of our investigation is that the light-curve data can be heavily averaged without compromising the accuracy of the inversion results. Even if the data are averaged to only two points per scale height, this introduces a numerical error of no more than $0.7 \%$ into the temperature profile for an isothermal atmosphere.

An area for future development of the inversion method would be the investigation of the correlated errors in the profiles, and specifically to derive formal errors for thermal gradients and other nonisothermal structure in the temperature profiles.
Application of this method to Pluto (with the caveat that the sharp drop in the light curve has no component due to extinction) yields an isothermal temperature profile down to a radius of $1210 \mathrm{~km}$, followed by a continuously increasing gradient at lower radii (the inversion ends at a radius of $\sim 1205 \mathrm{~km})$. Within the error bars, the profiles from the immersion and emersion sections of the light curve are the same. The maximum thermal gradient is $3.9 \pm 0.6 \mathrm{~K} \mathrm{~km}^{-1}$ (at $\sim 93 \mathrm{~K}$ ), which provides an additional constraint on radiative-conductive models.

For Triton, our method yields thermal profiles controlled by thermal conduction above altitudes of $50-60 \mathrm{~km}$, consistent with theoretical models. Below this boundary, however, the thermal profile becomes nearly isothermal, with wavelike structures that differ between the immersion and emersion results.

Our inversion technique will allow us to learn more about Pluto and Triton when new high-quality data become available. While Triton is moving farther from the Galactic plane, Pluto is approaching it. Hence the occultation frequency for Triton will decline (McDonald \& Elliot 2000a), while that for Pluto will increase (McDonald \& Elliot 2000b). Important goals for observations of these events for Pluto are to (1) probe more deeply into Pluto's shadow in order to test the "deep troposphere" scenario and (2) obtain light curves at two or more well-spaced wavelengths in order to distinguish between the "haze" and clearatmosphere scenarios.

Over the years the following people contributed to the development of this inversion method within our group: A. S. Bosh, C. B. Olkin, P. Tracadas, and M. A. Agner. We thank L. A. Young for helpful discussions concerning the Pluto results, and K. B. Clancy for help with the manuscript. An anonymous referee provided extensive comments that have helped to improve the manuscript. Partial support for this work was provided by NSF grant AST 00-73447 and by NASA, through grants NAG 5-3940, NAG 5-10444, GO-07489, and GO-08105 (the latter two from the Space Telescope Science Institute, which is operated by AURA, Inc., under NASA contract NAS 5-26555). S. Q. was partially supported by the Lord Foundation under the auspices of the Undergraduate Research Opportunities Program at MIT.

\title{
APPENDIX
}

\section{DEFINITIONS OF SYMBOLS}

\author{
Symbol Definition (First Appearance) \\ $A(r, b) \quad$ Asymptotic power series in the parameters $r$ and $b$ derived in Paper I ( $\S 3.3$, eq. [63]) \\ a Power-law index for molecular weight variation used in Paper I (set to zero here) ( $\$ 3.3$, after eq. [59]) \\ $a_{1} \quad$ Asymptotic distance of the undeflected ray from the center of mass used by FKE71 ( $\$ 2.3$, after eq. [16]) \\ $B_{p} \quad$ Short notation for $B_{p}\left(r_{b}, r\right)$ evaluated at $r_{i+1 / 2}(\S 4.3$, first paragraph $)$ \\ $B_{p}\left(r_{b}, r\right) \quad$ Pressure boundary integral ( $\$ 3.1$, before eq. [36]) \\ $B_{\nu} \quad$ Short notation for $B_{\nu}\left(r_{b}, r\right)$ evaluated at $r_{i+1 / 2}(\S 4.3$, first paragraph $)$ \\ $B_{\nu}\left(r_{b}, r\right) \quad$ Refractivity boundary integral ( $\$ 3.1$, before eq. [35]) \\ $B(r, b) \quad$ Asymptotic power series in the parameters $r$ and $b$ derived in Paper I ( $\S 3.3$, eq. [66]) \\ $b \quad$ Power-law index for temperature used in Paper I ( $\S 3.3$, second paragraph) \\ $\operatorname{Cov}(x, y) \quad$ Covariance of $x$ and $y(\S 4.1$, first paragraph) \\ $D$ \\ Distance between the observer and the occulting body (see Fig. 1) ( $\S 2.2$, first paragraph) \\ $f$ \\ Generic function ( $\$ 4.1$, first paragraph)
}


G Universal gravitational constant (§ 2.4, eq. [21])

$g\left(r^{\prime}\right) \quad$ Gravitational acceleration $(\S 2.4$, eq. [19])

$H \quad$ Atmospheric pressure scale height ( $\S 6.3$, before eq. [99])

$H_{h} \quad$ Atmospheric pressure scale height at half-light ( $\$ 3.3$, after eq. [60])

$H_{i+1 / 2} \quad$ Short notation for $H\left(r_{b}, r_{i+1 / 2}\right)(\S 3.2$, eq. [58])

$H\left(r_{b}, r_{i+1 / 2}\right) \quad$ Scale height at the lower boundary of a shell (§3.2, eq. [58])

$H(r) \quad$ Scale height as a function of radius ( $\$ 2.5$, after eq. [30])

$I_{p}\left(r_{b}, r\right) \quad$ Pressure integral over the region of atmosphere below $r_{b}(\S 3.1$, before eq. [37])

$I_{p}(r) \quad$ Pressure integral as a function of radius ( $\S 2.5$, after eq. [28])

$I_{\nu}\left(r_{b}, r\right) \quad$ Refractivity integral over the region of atmosphere below $r_{b}(\S 3.1$, before eq. [27])

$I_{\nu}(r) \quad$ Refractivity integral as a function of radius ( $\$ 2.5$, first paragraph)

$i \quad$ Shell index ( $\$ 3$, before eq. [32])

$i_{b} \quad$ Boundary index (first point in the inversion) ( $(3.1$, second paragraph)

$i_{\max } \quad$ Number of averaged light-curve points ( $(3$, first paragraph)

$j \quad$ Summation index (§ 4.1, before eq. [70])

$k$

$L \quad$ Loschmidt's number (\$2.3, eq. [17])

$M_{\mathrm{eff}} \quad$ Effective mass of a rotating occulting body $(\S 7.8$, second paragraph)

$M_{p} \quad$ Mass of occulting body ( $\S 2.4$, eq. [21])

$m_{\mathrm{amu}} \quad$ Atomic mass unit (§2.4, eq. [19])

$N$

$N(r)$

$n_{i+1 / 2}$

$n\left(r_{b}, r_{i+1 / 2}\right)$

$n(r)$

$p$

$p_{i+1 / 2}$

$p\left(r_{b}, r_{i+1 / 2}\right)$

$p(r)$

Number of fitted parameters for an atmospheric model describing the boundary region ( $\S 4.1$, before eq. [70])

$q_{N}$

$R \quad$ Ideal gas constant $(\S 2.5$, eq. [31])

Index of refraction of the atmosphere ( $\S 2.3$, second paragraph)

Short notation for $n\left(r_{b}, r_{i+1 / 2}\right)(\S 3.2$, eq. [55])

Number density at the lower boundary of a shell (§3.2, eq. [55])

Number density as a function of radius $(\S 2.3$, before eq. [16])

$r$

Atmospheric pressure ( $\S 10.1$, seventh paragraph)

Short notation for $p\left(r_{b}, r_{i+1 / 2}\right)(\S 3.2$, eq. [56])

Pressure at the lower boundary of a shell (§ 3.2, eq. [56])

Pressure as a function of radius ( $\S 2.4$, first paragraph)

Generic argument for a function (§ 4.1, first paragraph)

Ideal gas constant $(\S 2.5$, eq. [31])

Radial coordinate in the occulting body's atmosphere (Fig. 1); also minimum radius within the atmosphere of the occulting body for a given light ray ( 2.2 , first paragraph)

$r_{b} \quad$ Closest-approach radius of a light ray that corresponds to $y_{b}(\S 3.1$, second paragraph)

$r_{c} \quad$ Radius of curvature of a large-planet limb ( $\$ 7.8$, second paragraph)

$r_{\text {eff }} \quad$ Effective atmospheric radius in rotating body $(\S 7.8$, second paragraph)

$r_{h} \quad$ Half-light radius ( $\$ 3.3$, first paragraph)

$r_{i} \quad$ Radius at the middle of the $i$ th shell ( $(3.2$, eq. [46])

$r_{i+1 / 2} \quad$ Radius at the boundary of a shell that corresponds to $y_{i+1 / 2}$ (§ 3.2, before eq. [44])

$r_{01} \quad$ Minimum radius that the deflected ray passes from the center of mass used by FKE71 ( $\$ 2.3$, after eq. [16])

$r^{\prime} \quad$ Variable of integration ( $(2.3$, eq. [6])

$r^{\prime \prime} \quad$ Variable of integration $(\S 2.3$, before eq. [10])

$S_{p} \quad$ Short notation for $S_{p}\left(r_{b}, r_{i+1 / 2}\right)(\S 4.3$, first paragraph)

$S_{p}\left(r_{b}, r_{i+1 / 2}\right) \quad$ Summation approximation for $I_{p}\left(r_{b}, r\right)(\S 3.2$, before eq. [52] $)$

$S_{\nu} \quad$ Short notation for $S_{\nu}\left(r_{b}, r_{i+1 / 2}\right)(\S 4.3$, first paragraph)

$S_{\nu}\left(r_{b}, r_{i+1 / 2}\right) \quad$ Summation approximation for $I_{\nu}\left(r_{b}, r\right)$ (§ 3.2, before eq. [52])

$(\mathrm{S} / \mathrm{N})_{H} \quad$ Signal-to-noise ratio per scale height ( $\S 6.3$, first paragraph)

$T_{h} \quad$ Temperature at $r_{h}(\S 3.3$, second paragraph $)$

$T_{i+1 / 2} \quad$ Short notation for $T\left(r_{b}, r_{i+1 / 2}\right)(\S 3.2$, eq. [57])

$T\left(r_{b}, r_{i+1 / 2}\right) \quad$ Temperature at the lower boundary of a shell (§ 3.2, eq. [57])

$T(r) \quad$ Temperature as a function of radius $(\S 2.5$, before eq. [30])

$t_{i} \quad$ Midtime of each integration interval ( $\$ 3$, first paragraph)

$x \quad$ Coordinate along the rays of incident starlight (\$ 2.3, eq. [6])

$x_{i} \quad$ Generic quantity associated with the midvalue for the $i$ th atmospheric shell ( $\S 4.1$, before eq. [72])

$x_{i+1 / 2} \quad$ Generic quantity associated with the lower boundary value for the $i$ th shell ( $\$$ 4.1, before eq. [72])

$y$

$y_{b}$

$y_{i}$

$y_{i+1 / 2}$

$y(t)$

Coordinate in the observer plane perpendicular to a line in the direction of the star ( $\$ 2.2$, first paragraph)

Value of $y$ at the beginning of the inversion ( $\$ 3.1$, second paragraph)

The $y$-value associated with $\phi_{i}(\S 3$, first paragraph)

Values of $y$ at the boundary of the shells ( $\{3$, eq. [33])

Value of $y$ as a function of time that specifies the path of the observer in the observer plane $(\S 2.2$, second paragraph)

$y^{\prime} \quad$ Variable of integration (\$ 2.2, eq. [4])

$z_{j+} \quad$ Ratio: $r_{j+1 / 2} / r_{i+1 / 2}(\S 3.2$, eq. [51])

$z_{j-} \quad$ Ratio: $r_{j-1 / 2} / r_{i+1 / 2}(\S 3.2$, eq. [51]) 
$\alpha \quad$ Refraction angle used by FKE71 ( $§ 2.3$, after eq. [16])

$\gamma \quad$ Variable used for simplification: $\gamma=r^{\prime \prime} / r(\S 2.4$, after eq. [25])

$\Delta \theta_{i} \quad$ Change in $\theta$ over the $i$ th shell ( $\$ 3.2$, before eq. [48])

$\Delta r_{i} \quad$ Change in $r$ over the $i$ th shell ( $\{3.2$, before eq. [45])

$\Delta t_{i} \quad$ Integration time for the $i$ th flux, $\phi_{i}(\S 3$, first paragraph $)$

$\Delta y_{i} \quad$ Change in $y$ over the $i$ th shell ( $\{3$, eq. [33])

$\zeta\left(x_{i}\right) \quad$ Operator used to calculate the standard deviation of the calculated quantity, $x_{i}(\S 4.1$, before eq. [72])

$\theta_{i \pm 1 / 2} \quad$ Values of $\theta$ at the boundary of a shell ( $\$ 3.2$, before eq. [47])

$\theta(r)$

Angle through which the light ray is refracted by the atmosphere, as a function of radius ( $\S 2.2$, first paragraph)

$\theta_{b}(r) \quad$ Refraction angle for a light ray with a closest-approach radius $r$; defined for $r \geq r_{b}$ (§ 3.1, second paragraph)

$\lambda \quad$ Wavelength of starlight ( $\$$ 7.5.2, first paragraph)

$\lambda_{h} \quad$ Ratio of gravitational potential energy at the half-light radius to $k T_{h}(\S 3.3$, before eq. [60])

$\lambda_{\text {hi }} \quad$ "Equivalent isothermal " $\lambda$ at the half-light radius (see Paper I) ( $\S 3.3$, before eq. [62])

$\lambda(r) \quad$ Ratio of gravitational potential energy to $k T$ as a function of $r$ (§3.3, before eq. [61])

$\mu \quad$ Mean molecular weight of atmosphere (§ 2.4, eq. [22])

$\mu(r) \quad$ Molecular weight as a function of radius ( $\S 2.4$, first paragraph)

$\mu\left(r_{01}\right) \quad$ Index of refraction used by FKE71 ( $\$ 2.3$, after eq. [16])

$\nu_{h} \quad$ Refractivity at half-light $(\S 3.3$, before eq. [65])

$\nu_{i+1 / 2} \quad$ Short notation for $\nu\left(r_{b}, r_{i+1 / 2}\right)(\S 3.2$, eq. [54] $)$

$\nu_{\text {STP }} \quad$ Refractivity in at standard temperature and pressure for a homogeneous atmosphere ( $\$ 2.3$, eq. [18])

$\nu_{\mathrm{STP}}(r) \quad$ Refractivity at standard temperature and pressure for an inhomogeneous atmosphere ( $\S 2.3$, before eq. [17])

$\nu\left(r_{b}, r_{i+1 / 2}\right) \quad$ Refractivity at the lower boundary of a shell (§ 3.2, eq. [54])

$\nu(r) \quad$ Refractivity of the atmosphere as a function of radius $r$ ( $\$ 2.3$, after eq. [11])

$\rho \quad$ Symbol for $|y|$ used in Paper I ( $\$ 2.2$, second paragraph)

$\rho_{j k} \quad$ Correlation coefficient for the $j$ th and $k$ th parameters in a least-squares fit ( $\$ 4.1$, first paragraph)

$\sigma_{q, \text { symbol }}^{2}$ Contribution to the variance in "symbol" arising from the boundary condition: $\sigma_{q, r}^{2}, \sigma_{q, p}^{2}$, etc. ( $\S 4.2$, before eq. [77])

$\sigma_{\phi, \text { symbol }}^{2}$

Contribution to the variance in "symbol" arising from the flux summation terms in the inversion: $\sigma_{\phi, r}^{2}, \sigma_{\phi, p}^{2}$, etc. $(\S 4.2$, before eq. [77])

$\sigma($ symbol $) \quad$ Standard deviation of "symbol": $\sigma\left(\phi_{i}\right), \sigma\left(r_{b}\right)$, etc. ( $\$ 3$, first paragraph)

$\sigma^{2}$ (symbol) Variance of "symbol ": $\sigma^{2}\left(\phi_{k}\right), \sigma^{2}\left(r_{b}\right)(\S 4.1$, eq. [72])

$\phi_{i}$

$\phi(t)$

$\phi(y)$

$\phi_{\text {cyl }}(y)$

Set of normalized stellar fluxes collected during an occultation ( $\$ 3$, first paragraph)

Normalized stellar flux as a function of time, $t$ ( 2.2 , second paragraph)

Normalized stellar flux as a function of observer-plane coordinate, $y(\S 2.2$, second paragraph)

Stellar flux that would be refracted by a cylindrical (rather than spherical) atmosphere ( $\$ 2.2$, second paragraph)

Acton, F. S. 1970, Numerical Methods That Work (New York: Harper \& Row)

Aki, K., \& Richards, P. G. 1980, Quantitative Seismology, Vol. 2 (San Francisco: Freeman)

Bevington, P. R., \& Robinson, D. K. 1992, Data Reduction and Error Analysis for the Physical Sciences (2d ed.; New York: McGraw-Hill)

Born, M., \& Wolf, E. 1964, Principles of Optics (2d rev. ed.; New York: MacMillan)

Bosh, A. S., Young, L. A., Elliot, J. L., Hammel, H. B., \& Baron, R. L. 1992, Icarus, 95, 319

Broadfoot, A. L., et al. 1989, Science, 246, 1459

Brown, G. N., Jr., \& Ziegler, W. T. 1980, Adv. Cryogenic Eng., 25, 662

Buie, M. W., et al. 2002, BAAS, 34, 877

Chamberlain, D. M., \& Elliot, J. L. 1997, PASP, 109, 1170

Cooray, A. R., \& Elliot, J. L. 2003, ApJ, 587, L121

Cruikshank, D. P., Roush, T. L., Owen, T. C., Geballe, T. R., de Bergh, C.,

Schmitt, B., Brown, R. H., \& Bartholomew, M. J. 1993, Science, 261, 742

Dunham, E. W., Baron, R. L., Elliot, J. L., Vallerga, J. V., Doty, J. P., \&

Ricker, G. R. 1985, PASP, 97, 1196

Elliot, J. L., et al. 1993, AJ, 106, 2544

Elliot, J. 2003, Nature, in press

Elliot, J. L., Dunham, E. W., Bosh, A. S., Slivan, S. M., Young, L. A., Wasserman, L. H., \& Millis, R. L. 1989, Icarus, 77, 148

Elliot, J. L., French, R. G., Dunham, E., Gierasch, P. J., Veverka, J., Church, C., \& Sagan, C. 1977, ApJ, 217, 661

Elliot, J. L., French, R. G., Meech, K. J., \& Elias, J. H. 1984, AJ, 89, 1587

Elliot, J. L., et al. 1998, Nature, 393, 765 2000a, Icarus, 148,347

Elliot, J. L., Rages, K., \& Veverka, J. 1976, ApJ, 207, 994

Elliot, J. L., Stansberry, J. A., Olkin, C. B., Agner, M. A., \& Davies, M. E. 1997, Science, 278, 436

Elliot, J. L., Strobel, D. F., Zhu, X., Stansberry, J. A., Wasserman, L. H., \& Franz, O. G. 2000b, Icarus, 143, 425

Elliot, J. L., \& Veverka, J. 1976, Icarus, 27, 359

Elliot, J. L., \& Young, L. A. 1992, AJ, 103, 991 (Paper I)

\section{EFERENCES}

Eshleman, V. R. 1989, Icarus, 80, 439

Eshleman, V. R., \& Gurrola, E. M. 1993, Icarus, 105, 298

Fairall, A. P. 1972, Nature, 236, 342

Fjeldbo, G., \& Eshleman, V. R. 1965, J. Geophys. Res., 70, 3217

Fjeldbo, G., Kliore, A. J., \& Eshleman, V. R. 1971, AJ, 76, 123 (FKE71)

Foust, J. A., Elliot, J. L., Olkin, C. B., McDonald, S. W., Dunham, E. W., Stone, R. P. S., McDonald, J. S., \& Stone, R. C. 1997, Icarus, 126, 362

French, R. G., Elliot, J. L., \& Gierasch, P. J. 1978, Icarus, 33, 186

French, R. G., Elliot, J. L., Sicardy, B., Nicholson, P., \& Matthews, K. 1982, Icarus, 51, 491

French, R. G., \& Lovelace, R. V. E. 1983, Icarus, 56, 122

Hansen, C. J., \& Paige, D. A. 1992, Icarus, 99, 273

Hilbert, B. N. 2001, Master's thesis, MIT

Hubbard, W. B., Hunten, D. M., Dieters, S. W., Hill, K. M., \& Watson, R. D. 1988, Nature, 336, 452

Hubbard, W. B., Hunten, D. M., Reitsema, H. J., Brosch, N., Nevo, Y. Carreira, E., Rossi, F., \& Wasserman, L. H. 1990a, Nature, 343, 353

Hubbard, W. B., \& Jokipii, J. R. 1977, Icarus, 30, 531

Hubbard, W. B., Jokipii, J. R., \& Wilking, B. A. 1978, Icarus, 34, 374

Hubbard, W. B., et al. 1997, Icarus, 130, 404

Hubbard, W. B., Yelle, R. V., \& Lunine, J. I. 1990b, Icarus, 84, 1

Ingersoll, A. P. 1990, Nature, 344, 315

Kovalevsky, J., \& Link, F. 1969, A\&A, 2, 398

Krasnopolsky, V. A., \& Cruikshank, D. P. 1999, J. Geophys. Res., 104, 21979

Lellouch, E. 1994, Icarus, 108, 255

McDonald, S. W., \& Elliot, J. L. 1995, AJ, 109, 1352 $.2000 \mathrm{a}, \mathrm{AJ}, 119,936$ 2000b, AJ, 119, 1999 (erratum 120, 1599)

Millis, R. L., et al. 1993, Icarus, 105, 282

Mink, D. J., \& Klemola, A. 1985, AJ, 90, 1894

Narayan, R., \& Hubbard, W. B. 1988, ApJ, 325, 503

Nather, R. E., \& Evans, D. S. 1970, AJ, 75, 575

Null, G. W., \& Owen, W. M., Jr. 1996, AJ, 111, 1368

Olkin, C. B., et al. 1997, Icarus, 129, 178

Olkin, C. B., Wasserman, L. H., \& Franz, O. G. 2003, Icarus, in press 
Owen, T. C., et al. 1993, Science, 261, 745

Person, M. J., Elliot, J. L., Pate, J., Glass, I., Stone, R. C., Morzinski, K. M., \& Dunham, E. W. 2001, BAAS, 33, 1130

Roques, F., Moncuquet, M., \& Sicardy, B. 1987, AJ, 93, 1549

Roques, F., et al. 1994, A\&A, 288, 985

Sicardy, B., et al. 2003, Nature, in press

$$
\text { 1999, Icarus, 142, } 357
$$

Smith, B. A., et al. 1989, Science, 246, 1422

Spencer, J. R., \& Moore, J. M. 1992, Icarus, 99, 261

Stansberry, J. A., Lunine, J. I., Hubbard, W. B., Yelle, R. V., \& Hunten, D. M. 1994, Icarus, 111, 503

Stansberry, J. A., Lunine, J. I., \& Tomasko, M. G. 1989, Geophys. Res. Lett., 16, 1221

Stansberry, J. A., \& Yelle, R. V. 1999, Icarus, 141, 299

Strobel, D. F., Cheng, A. F., Summers, M. E., \& Strickland, D. J. 1990, Geophys. Res. Lett., 17, 1661

Strobel, D. F., \& Summers, M. E. 1995, in Neptune and Triton, ed. D. P. Cruikshank (Tucson: Univ. Arizona Press), 1107

Strobel, D. F., Zhu, X., Summers, M. E., \& Stevens, M. H. 1996, Icarus, 120,266
Tryka, K. M., Brown, R. H., Cruikshank, D. P., Owen, T. C., Geballe, T. R., \& de Bergh, C. 1994, Icarus, 112, 513

Tyler, G. L., et al. 1989, Science, 246, 1466

van Belle, G. T. 1999, PASP, 111, 1515

Veverka, J., Elliot, J., Sagan, C., Wasserman, L., \& Liller, W. 1972, Nature, 240,344

Wasserman, L. H., \& Veverka, J. 1973, Icarus, 20, 322

Wolfram, S. 1999, The Mathematica Book (4th ed.; New York: Cambridge Univ. Press)

Yelle, R. V.. \& Lunine, J. I. 1989, Nature, 339, 288

Yelle, R. V., Lunine, J. I., \& Hunten, D. M. 1991, Icarus, 89, 347

Yelle, R. V., Lunine, J. I., Pollack, J. B., \& Brown, R. H. 1995, in Neptune and Triton, ed. D. P. Cruikshank (Tucson: Univ. Arizona Press), 1031

Young, A. T. 1967, AJ, 72, 747

. 1976, Icarus, 27, 335

Young, E. F., \& Binzel, R. P. 1994, Icarus, 108, 219

Young, L. A. 1994, Ph.D. thesis, MIT

Young, L. A., Elliot, J. L., Tokunaga, A., de Bergh, C., \& Owen, T. 1997, Icarus, 127, 258 